\title{
Relationship of stereochemical and skeletal diversity of small molecules to cellular measurement space
}

\author{
Young-kwon Kim, Midori A. Arai, Takayoshi Arai, Julia O. Lamenzo, Elton F. Dean III, \\ Nick Patterson, Paul A. Clemons*, and Stuart L. Schreiber* \\ Howard Hughes Medical Institute, \\ Department of Chemistry and Chemical Biology, \\ The Eli \& Edythe Broad Institute, \\ Program in Chemical Biology \\ Harvard University, Cambridge, Massachusetts 02138 USA

\section{Supporting Information}

I. General methods

II. Preliminary studies in solution-phase

III. Synthesis of building blocks

IV. Parallel library synthesis and analysis

V. Assay methods, error modeling and data analysis

VI. Statistical analysis

VII. NMR spectra 


\section{General methods}

Materials. Commercially available reagents were purchased from Aldrich Chemical Co., Fluka Chemical Corp., and Strem and used without further purification unless otherwise noted. All solvents were dispensed from a solvent purification system that passes solvents through packed columns (THF, diethyl ether, acetonitrile and methylene chloride: dry neutral alumina; hexane, benzene and toluene: dry neutral alumina and Q5 reactant; DMF: activated molecular sieves). ${ }^{1}$ Triethylamine, diisopropylamine, pyridine, and 2,6-lutidines were distilled under nitrogen from $\mathrm{CaH}_{2}$. Macrobeads were prepared at ICCB (Institute of Chemistry and Cell Biology, Harvard University) as previously published. ${ }^{2}$

Solid-phase reactions. All solid-phase reactions were performed in oven dried glassware (including Wheaton vials fitted with Teflon-coated caps) with gentle mixing provided by Thermolyne labquake shaker (35 rotations per min) or a Vortex Genie-2 vortexer or Thermolyne Roto Mix longitudinal orbital shaker. After the completion of the reaction, macrobeads were isolated by filtration in fritted columns on a Vac-Man laboratory Vacuum Manifold (Promega A7231) fitted with nylon three way stop cocks (BioRad 732-8107). Macrobeads were then washed and dried under air flow and/or house vac and/or high vac and/or lyophilizer.

Solution-phase reactions. All reactions were performed in oven-or frame-dried glassware under positive nitrogen or argon pressure unless otherwise noted. The progress of the reactions were monitored by analytical thin-layer chromatography using indicated eluents on E. Merck silica gel 60 F254 plates (thickness: $0.25 \mathrm{~mm}$ ). Visualization was by the quenching of UV fluorescence $\left(\lambda_{\max }=254 \mathrm{~nm}\right)$ and/or by staining with ceric ammonium molybdate or anisaldehyde solution with or without the treatment of $20 \%$ (w/w) Iodine slurry in silica gel.

Purification and analysis. Flash chromatography was performed using indicated eluents on E. Merck silica gel 60. Medium pressure liquid chromatography (MPLC) was performed CombiFlash 
companion (ISCO) with pre-packed silica gel column with indicated eluents gradients. Infrared spectra were collected as a thin film on $\mathrm{NaCl}$ plates on a Nicolet 5PC FTIR spectrometer with internal referencing. ${ }^{1} \mathrm{H}$ nuclear magnetic resonance spectra were recorded on Varian Unity/Inova 500 (500MHz). ${ }^{1} \mathrm{H}$ data are reported as follows; chemical shift (multiplicity, integration, $J=$ coupling constants in Hertz $(\mathrm{Hz})$, annotation). Chemical shifts are recorded in parts per million downfield from tetramethylsilane $(\delta$ scale) and referenced from residual protium in the NMR solvent. Multiplicity is assigned as follows; $\mathrm{s}=$ singlet, $\mathrm{d}=$ doublet, $\mathrm{t}=$ triplet, $\mathrm{q}=$ quartet, quint $=$ quintet, $\mathrm{sext}=$ sextet, $\mathrm{m}=$ multiplet, $\mathrm{c}=$ complex $($ due to overlap of multiple peaks with different chemical shifts) and $\mathrm{br}=$ broadened. ${ }^{3(\mathrm{a}) \text {,(b) }{ }^{13} \mathrm{C} \text { magnetic }}$ resonance spectra were recorded on Varian Unity/Inova $400(101 \mathrm{MHz}) .{ }^{13} \mathrm{C}$ chemical shifts are reported in parts per million downfield from tetramethylsilane ( $\delta$ scale) and referenced to carbon resonances in the NMR solvent. All ${ }^{13} \mathrm{C}$ spectra were determined with broadband ${ }^{1} \mathrm{H}$ decoupling. Solid-phase MAS ${ }^{1} \mathrm{H}$ NMR were obtained in $\mathrm{CD}_{2} \mathrm{Cl}_{2}$ on a Varian Inova $600(600 \mathrm{MHz})$ fitted with a magic angle spinning nanoprobe. Reverse phase LCMS data was obtained from a Gilson/Finnigan LC/MS system. Chromatography was performed on a SymmetryShield ${ }^{\mathrm{TM}} \mathrm{RP}_{8}, 3.5 \mu \mathrm{M}, 4.6 \times 100 \mathrm{~mm}$ column (Waters corporation, Batch \# 111) under the conditions with flow rate of $1 \mathrm{~mL} / \mathrm{min}$ and a $10 \mathrm{~min}$ gradient from 5 $\%$ to $95 \% \mathrm{CH}_{3} \mathrm{CN}$ in water, constant $0.1 \%$ formic acid. The detection was performed with UV detectors at $214 \mathrm{~nm}$ and $254 \mathrm{~nm}$ and with Finnigan Aqa MS with Electron spray positive mode (ES+). Highresolution mass spectra (HRMS) were obtained at the mass spectrometry facility at Harvard University. 


\section{Preliminary studies in solution-phase}
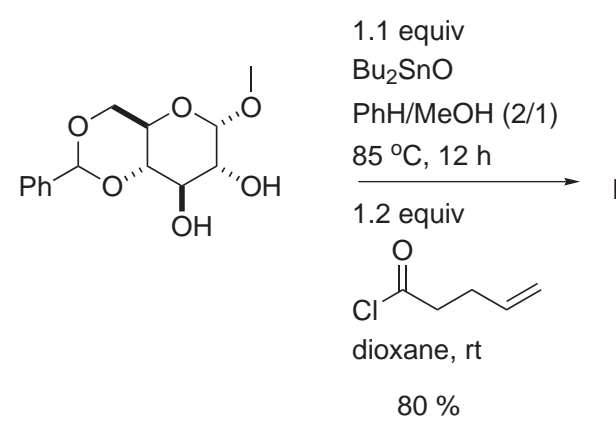
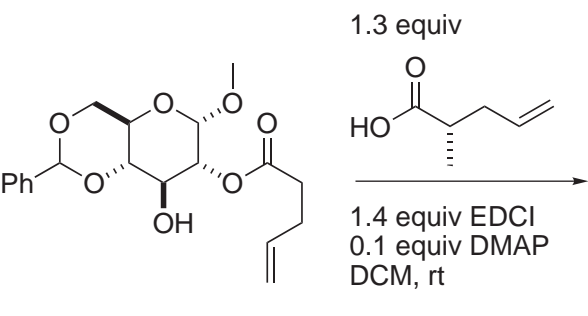

$95 \%$<smiles>C=CCCC(=O)O[C@H]1C(OC)O[C@@H]2COC(c3ccccc3)O[C@@H]2[C@H]1OC(=O)[C@H](C)CC=C</smiles>

1. $40 \%$ aq. $\mathrm{AcOH}$ $80^{\circ} \mathrm{C}, 10 \mathrm{~h}$

2. 1.5 equiv $\mathrm{TrCl}$ 1 equiv DMAP Pyr, $80^{\circ} \mathrm{C}, 15 \mathrm{~h}$

$78 \%$<smiles>C=CCCC(=O)O[C@@H]1C(OC(=O)[C@@H](C)CC=C)[C@H](O)C(CO[Te])O[C@@H]1OC</smiles>
1.3 equiv

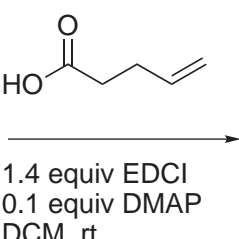

$90 \%$<smiles>C=CCCC(=O)OC1[C@H](OC(=O)CCC=C)[C@H](OC)O[C@H](CO)[C@H]1OC(=O)CCC=C</smiles>

1a<smiles>COC1OC(CO)C(O)C(O)C1O</smiles>

1. 1.5 equiv $\mathrm{TrCl}$ 1 equiv DMAP

Pyr, $80^{\circ} \mathrm{C}, 15 \mathrm{~h}$<smiles>C=CCCC(=O)O</smiles>

1.4 equiv EDCI

0.1 equiv DMAP

DCM, rt

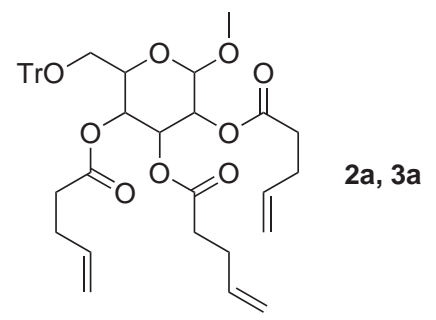

Scheme 1. Preparations of substrate 1a, 2a, 3a 


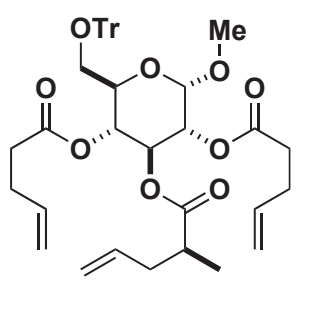

$1 \mathrm{a}$

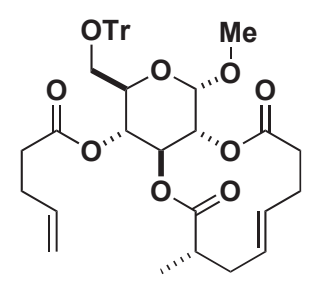

$1 \mathrm{~b}$

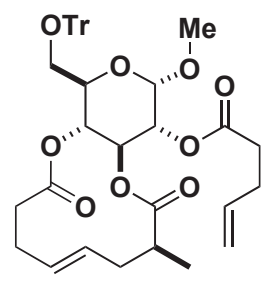

$1 \mathrm{c}$

To a solution of triene 1a (35 mg, $0.5 \mathrm{mmol})$ in $\mathrm{CH}_{2} \mathrm{Cl}_{2}(250 \mathrm{~mL})$ was added a portion of Grubbs' catalyst $(27.5 \mathrm{mg}, 0.025 \mathrm{mmol}, 5 \mathrm{~mol} \%)$. The reaction mixture was stirred at $40{ }^{\circ} \mathrm{C}$ for $90 \mathrm{~min}$ and concentrated. The concentrate was filtered through silica column $(20 \mathrm{~g})$ saturated with TEA with diethyl ether. The filtrate was concentrated and purified by flash chromatography (120 g of silica gel, Hex/EtOAc: $5 / 1)$ to yield $\mathbf{1 b}(153 \mathrm{mg}, 45 \%)$ and $\mathbf{1 c}(137 \mathrm{mg}, 40 \%)$.

\section{Glc_triene_product_1b}

$\mathbf{R}_{\mathbf{f}}=0.6(\mathrm{~A})$

${ }^{1}$ H NMR (500 MHz, $\left.\mathrm{CDCl}_{3}\right)$ : $\delta 1.10$ (d, 3H, $\left.J=7.0\right), 1.96-2.49$ (c. 8H), 2.59-2.81 (c, 3H), 3.05 (dd, 1H, $J=5, J=10), 3.21(\mathrm{dd}, 1 \mathrm{H}, J=7, J=10), 3.45$ (s, 3H), $4.04(\mathrm{dd}, 1 \mathrm{H}, J=5, J=7), 4.84$ (dd, $1 \mathrm{H}, J=3.5$, $J=10.0), 4.94$ (d, 1H, $J=3.5), 5.00$ (app t, 1H, $J=10.0$ ), 5.10 (c, 2H), 5.62 (app t, 1H, $J=10$ ), 5.78-5.91 (c, $1 \mathrm{H}), 7.22-7.41(\mathrm{c}, 15 \mathrm{H})$

${ }^{13}$ C NMR (101 MHz, $\left.\mathrm{CDCl}_{3}\right): \delta 16.1,28.3,28.4,28.7,33.0,33.4,55.0,62.5,66.4,69.3,69.7,70.0,86.5$, 98.3, 115.4, 115.4, 115.8, 126.0 (3), 127.7 (3), 128.7 (3), 136.2, 136.3, 136.3, 143.7, 171.3, 171.9, 172.1

IR (NaCl): 3151 (w), $2944(\mathrm{~m}), 1748(\mathrm{~s}), 1613(\mathrm{w}), 1270(\mathrm{~m}), 1166(\mathrm{w}), 757(\mathrm{w}) \mathrm{cm}^{-1}$

HRMS-ESI $(\mathrm{m} / \mathrm{z})$ : $\left[\mathrm{M}+\mathrm{NH}_{4}\right]^{+}$calcd for $\mathrm{C}_{40} \mathrm{H}_{49} \mathrm{NO}_{9}$ 686.3329; found, 686.3323 .

\section{Glc_triene_product_1c}

$\mathbf{R}_{\mathbf{f}}=0.45$ (A)

${ }^{1}$ H NMR $\left(500 \mathrm{MHz}, \mathrm{CDCl}_{3}\right): \delta 1.14(\mathrm{~d}, 3 \mathrm{H}, J=7.0), 2.17-2.53(\mathrm{c}, 9 \mathrm{H}), 2.65-2.81(\mathrm{c}, 2 \mathrm{H}), 3.67(\mathrm{t}, 1 \mathrm{H}, J$ $=8), 3.32(\mathrm{dd}, 1 \mathrm{H}, J=6.5, J=8), 3,41(\mathrm{~s}, 3 \mathrm{H}), 4.05(\mathrm{~d}, 1 \mathrm{H}, J=6.5), 4.98(\mathrm{dd}, 1 \mathrm{H}, J=3.5, J=10.0), 4.98$ (t, $1 \mathrm{H}, J=10.0), 4.95-5.07$ (c, 2H), 5.44-5.52 (c, 2H), 5.66 (app t, $1 \mathrm{H}, J=10.0), 5.71-5.84$ (c, 1H), 7.22$7.41(\mathrm{c}, 15 \mathrm{H})$

${ }^{13}$ C NMR (101 MHz, $\left.\mathrm{CDCl}_{3}\right): \delta 15.3,26.7,28.1,28.7,32.7,33.3,34.1,54.7,61.7,67.5,67.6,86.3,96.6$, 115.0, 126.5 (3), 127.3 (3), 128.1 (3), 129.2, 130.2, 135.7, 143.1, 170.5, 171.8, 171.9

IR (NaCl): 2932 (w), 1746 (s), 1448 (w), 1164 (m), 1132 (w), 1083 (m), 979 (w) cm .

HRMS-ESI $(\mathrm{m} / \mathrm{z})$ : $[\mathrm{M}+\mathrm{Na}]^{+}$calcd for $\mathrm{C}_{40} \mathrm{H}_{44} \mathrm{NaO} 9$ 691.2883; found, 691.2880. 


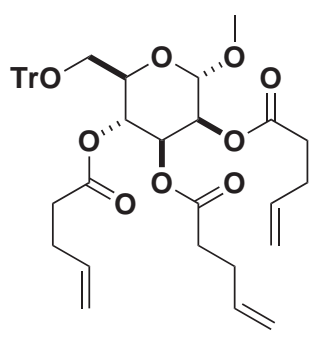

$2 \mathbf{a}$

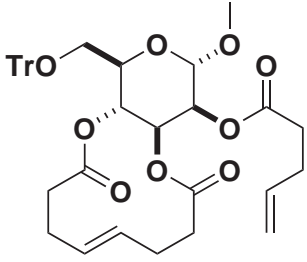

$2 b$

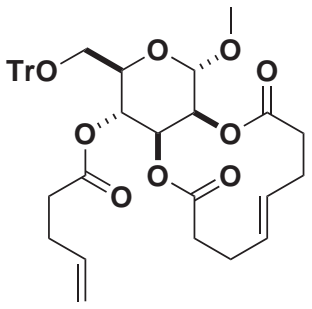

2c

To a solution of triene $\mathbf{2 a}(700 \mathrm{mg}, 1.0 \mathrm{mmol})$ in $\mathrm{CH}_{2} \mathrm{Cl}_{2}(500 \mathrm{~mL})$ was added a portion of Grubbs' catalyst $(57 \mathrm{mg}, 0.05 \mathrm{mmol}, 5 \mathrm{~mol} \%)$. The reaction mixture was stirred at $40{ }^{\circ} \mathrm{C}$ for $90 \mathrm{~min}$ and concentrated. The concentrate was filtered through silica column ( $20 \mathrm{~g})$ saturated with TEA with diethyl ether for $30 \mathrm{~min}$. The filtrate was concentrated and purified by flash chromatography (150 $\mathrm{g}$ of silica gel, Hex/EtOAc: $5 / 1)$ to yield $\mathbf{2 b}$ (541 mg, 79\%) and $\mathbf{2 c}$ (58 mg, $8 \%$ ).

\section{Man_product_2b}

$\mathbf{R}_{\mathbf{f}}=0.6(\mathrm{~A})$

${ }^{1}$ H NMR (500 MHz, $\mathrm{CDCl}_{3}$ ): $\delta$ 1.96- 2.49 (c. 12H), 3.10 (br s, 2H) 3.39 (s, 3H), 3.80 (m, 1H), 4.67 (d, $1 \mathrm{H}, J=1.5), 4.96$ (dd, $1 \mathrm{H}, J=1.5, J=10), 4.90-5.12$ (c, $2 \mathrm{H}), 5.10$ (dd, $1 \mathrm{H}, J=1.5, J=3.5), 5.20-5.31$ (c, $2 \mathrm{H}), 5.31(\mathrm{dd}, 1 \mathrm{H}, J=3.5, J=10), 5.77(\mathrm{ddd}, 1 \mathrm{H}, J=7, J=10, J=15), 7.22-7.41(\mathrm{c}, 15 \mathrm{H})$

${ }^{13}$ C NMR $\left(101 \mathrm{MHz}, \mathrm{CDCl}_{3}\right): \delta 28.3,28.4,28.7,33.0,33.2,33.4,55.0,62.5,66.4,69.3,69.7,70.0,86.5$, 98.3, 115.4, 115.4, 115.8, 126.9 (3), 127.7 (3), 128.7 (3), 136.2, 136.3, 136.3, 143.7, 171.3, 171.9, 172.1 IR (NaCl): 2944 (m), 1748 (s), 1613 (w), 1531 (m), 1270 (m), $1166(\mathrm{w}), 757$ (w) cm

HRMS-ESI $(\mathrm{m} / \mathrm{z}):\left[\mathrm{M}+\mathrm{NH}_{4}\right]^{+}$calcd for $\mathrm{C}_{39} \mathrm{H}_{46} \mathrm{NO} 9672.31726$; found, 672.3179.

\section{Man_product_2c}

$\mathbf{R}_{\mathbf{f}}=0.45(\mathrm{~A})$

${ }^{1}$ H NMR (500 MHz, $\left.\mathrm{CDCl}_{3}\right): \delta$ 1.95-2.60 (c, $\left.12 \mathrm{H}\right), 3.18$ (br d, 2H, $\left.J=4.5\right) 3.47$ (s, 3H), 3.87 (app dt, $1 \mathrm{H}, J=4.5, J=10), 4.74(\mathrm{~d}, 1 \mathrm{H}, J=1.5), 5.02-5.12(\mathrm{c}, 2 \mathrm{H}), 5.18(\mathrm{dd}, 1 \mathrm{H}, J=1.5, J=3.5), 5.29$ (app t, $1 \mathrm{H}, J=10), 5.26-5.33$ (c, $2 \mathrm{H}), 5.38$ (dd, $1 \mathrm{H}, J=3.5, J=10), 5.85$ (ddd, $1 \mathrm{H}, J=7, J=10, J=15), 7.22$ $7.41(\mathrm{c}, 15 \mathrm{H})$

${ }^{13}$ C NMR (101 MHz, $\left.\mathrm{CDCl}_{3}\right): \delta 28.3,28.4,28.7,33.0,33.1,33.4,55.0,62.5,66.4,69.3,69.7,70.0,86.5$, 98.3, 115.4, 115.4, 115.8, 126.9 (3), 127.7 (3), 128.7 (3), 136.2, 136.3, 136.3, 143.7, 171.3, 171.9, 172.1 IR (NaCl): 2932 (w), 1746 (s), 1448 (w), 1164 (m), 1132 (w), 1083 (m), 979 (w) cm . HRMS-ESI $(\mathrm{m} / \mathrm{z}):\left[\mathrm{M}+\mathrm{NH}_{4}\right]^{+}$calcd for $\mathrm{C}_{39} \mathrm{H}_{46} \mathrm{NO}_{9}$ 672.31726; found, 672.3176. 

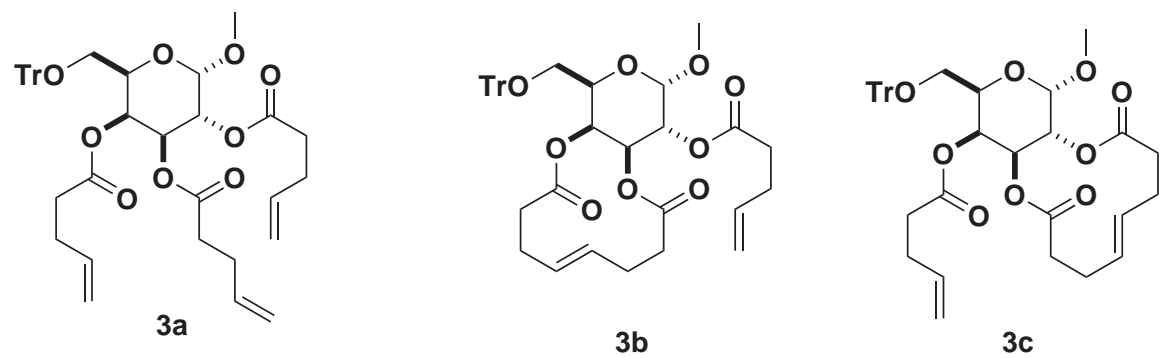

To a solution of triene 3a $(630 \mathrm{mg}, 0.9 \mathrm{mmol})$ in $\mathrm{CH}_{2} \mathrm{Cl}_{2}(450 \mathrm{~mL})$ was added a portion of Grubbs' catalyst (51 mg, $0.045 \mathrm{mmol}, 5 \mathrm{~mol} \%$ ). The reaction mixture was stirred at $40{ }^{\circ} \mathrm{C}$ for $90 \mathrm{~min}$ and concentrated. The concentrate was filtered through silica column (10 g) saturated with TEA with diethyl ether for $30 \mathrm{~min}$. The filtrate was concentrated and purified by flash chromatography (100 $\mathrm{g}$ of silica gel, Hex/EtOAc: $5 / 1$ ) to yield 3c (482 mg, 78\%) and 3b (54 mg, 9\%).

\section{Gal_product_3b}

$\mathbf{R}_{\mathbf{f}}=0.6(\mathrm{~A})$

${ }^{1}$ H NMR (500 MHz, $\left.\mathrm{CDCl}_{3}\right)$ : $\delta$ 2.17-2.49 (c, $\left.12 \mathrm{H}\right), 3.05$ (dd, $\left.1 \mathrm{H}, J=5, J=10\right), 3.21(\mathrm{dd}, 1 \mathrm{H}, J=7, J=$ 10), 3.45 (s, 3H), 4.04 (dd, 1H, $J=5, J=7), 4,94$ (d, 1H, $J=3.5$ ), 4.99-5.09 (c, 2H), 5.20 (dd, 1H, $J=$ $3.5, J=10.5$ ), 5.22- 5.50 (c, $2 \mathrm{H}), 5.29$ (dd, $1 \mathrm{H}, J=3.5, J=10.5$ ), 5,53 (br d, $1 \mathrm{H}, J=3.5$ ), 5.81 (ddd, $1 \mathrm{H}$, $J=7, J=10, J=15), 7.22-7.41(\mathrm{c}, 15 \mathrm{H})$

${ }^{13}$ C NMR $\left(101 \mathrm{MHz}, \mathrm{CDCl}_{3}\right): \delta 26.7,28.1,28.7,32.7,33.3,34.1,54.7,61.7,67.5,67.6,86.3,96.6$, 115.0, 126.5 (3), 127.3 (3), 128.1 (3), 129.2, 130.2, 135.7, 143.1, 170.5, 171.8, 171.9

IR (NaCl): 2926 (w), 1743 (s), 1448 (w), 1240 (w), 1140 (w), 1047 (m), $706(w) \mathrm{cm}^{-1}$.

HRMS-ESI $(\mathrm{m} / \mathrm{z}):\left[\mathrm{M}+\mathrm{NH}_{4}\right]^{+}$calcd for $\mathrm{C}_{39} \mathrm{H}_{46} \mathrm{NO} 9672.31726$; found, 672.3163.

\section{Gal_product_3c}

$\mathbf{R}_{\mathbf{f}}=0.45(\mathrm{~A})$

${ }^{1}$ H NMR (500 MHz, $\mathrm{CDCl}_{3}$ ): $\delta$ 2.17-2.49 (c, $\left.12 \mathrm{H}\right), 3.06$ (app t, $\left.1 \mathrm{H}, J=9.6\right) 3.43(\mathrm{~s}, 3 \mathrm{H}), 3.67$ (t, $1 \mathrm{H}, J=$ 8), $3.32(\mathrm{dd}, 1 \mathrm{H}, J=6.5, J=8), 3,41(\mathrm{~s}, 3 \mathrm{H}), 4.05$ (d, 1H, $J=6.5), 4.94$ (d, 1H, $J=2.0), 4.95-5.07$ (c, $4 \mathrm{H}), 5.12(\mathrm{dd}, 1 \mathrm{H}, J=2.0, J=10), 5.39$ (dd, $1 \mathrm{H}, J=2.0, J=10), 5,53$ (br d, $1 \mathrm{H}, J=4.0), 5.71-5.84$ (c, $3 \mathrm{H}), 7.22-7.41(\mathrm{c}, 15 \mathrm{H})$

${ }^{13}$ C NMR (101 MHz, $\left.\mathrm{CDCl}_{3}\right): \delta$ 26.8, 28.2, 28.9, 32.9, 33.2, 34.0, 54.5, 61.7, 67.6, 67.6, 86.3, 97.1, $114.8,126.3$ (3), $127.3(3), 128.4(3), 129.3,130.1,135.8,143.0,170.3,172.1172 .2$

IR (NaCl): 2940 (w), 1758 (s), 1370 (m), 1274 (s), 1095 (m), 1050 (w), 988 (m) cm .

HRMS-ESI $(\mathrm{m} / \mathrm{z}):\left[\mathrm{M}+\mathrm{NH}_{4}\right]^{+}$calcd for $\mathrm{C}_{39} \mathrm{H}_{46} \mathrm{NO} 9$ 672.31726; found, 672.3173. 


\section{Synthesis of building blocks}
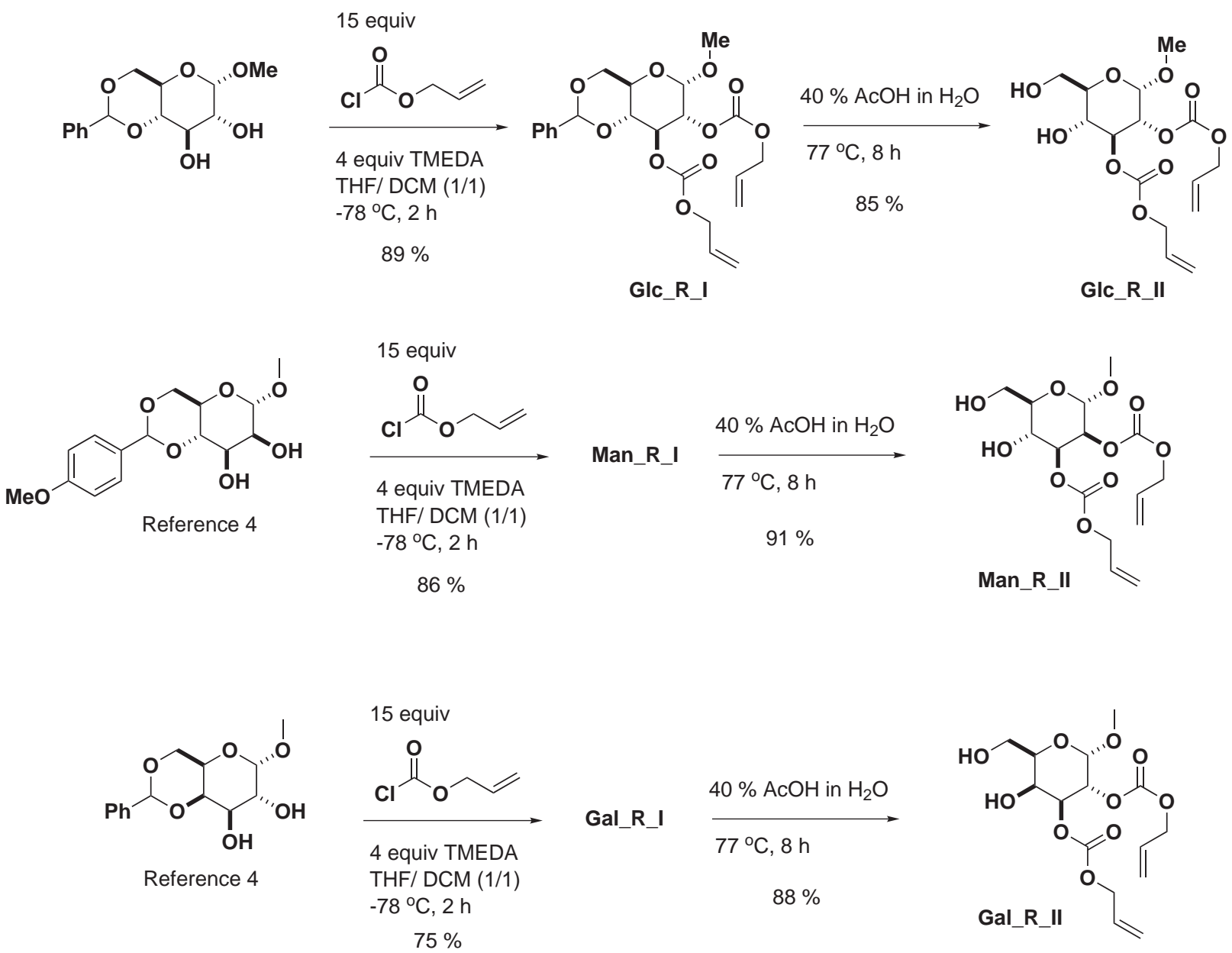

Scheme 2. Synthesis of 3,4-bis-protected carbohydrate derivatives 

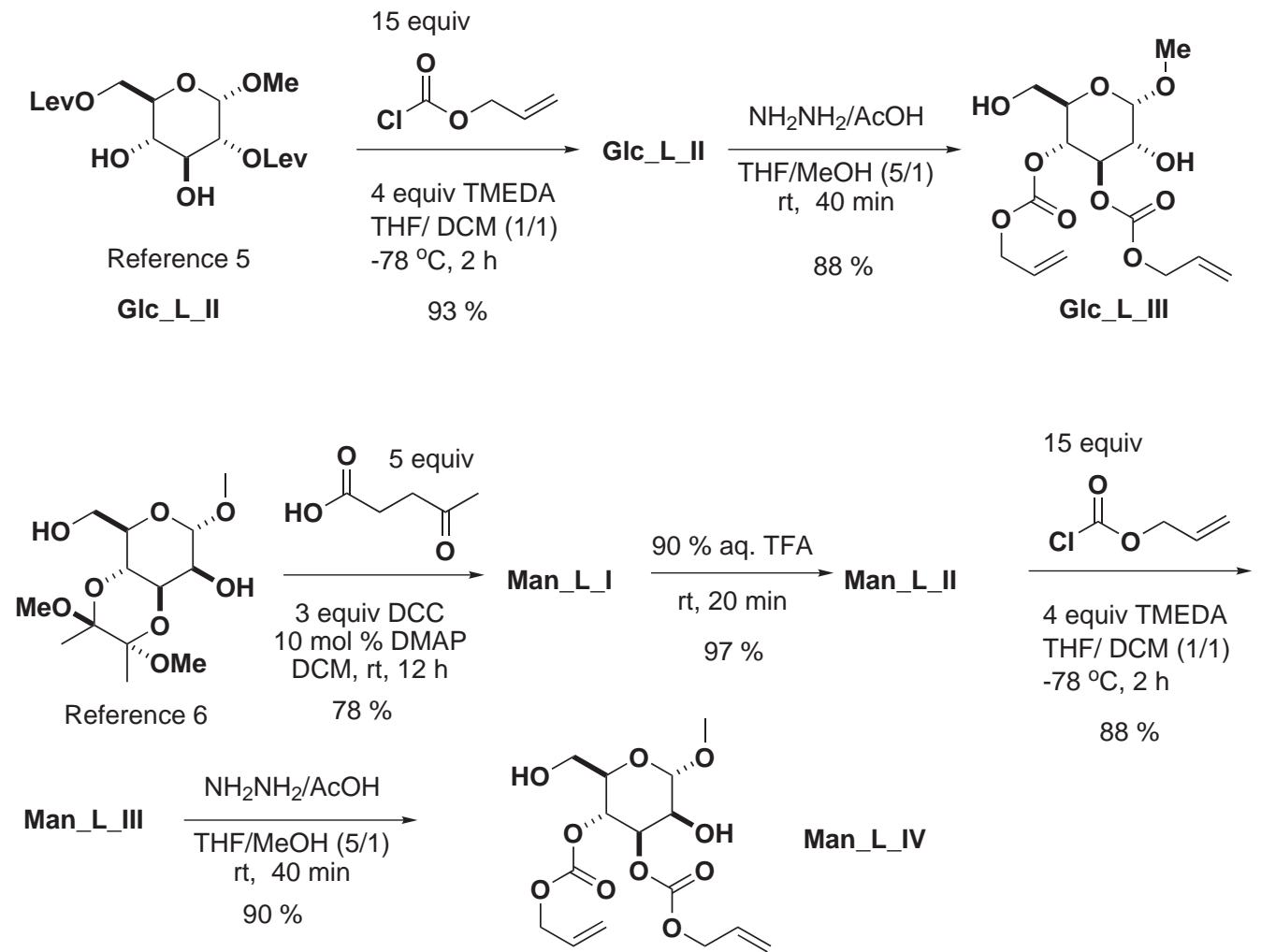

$88 \%$

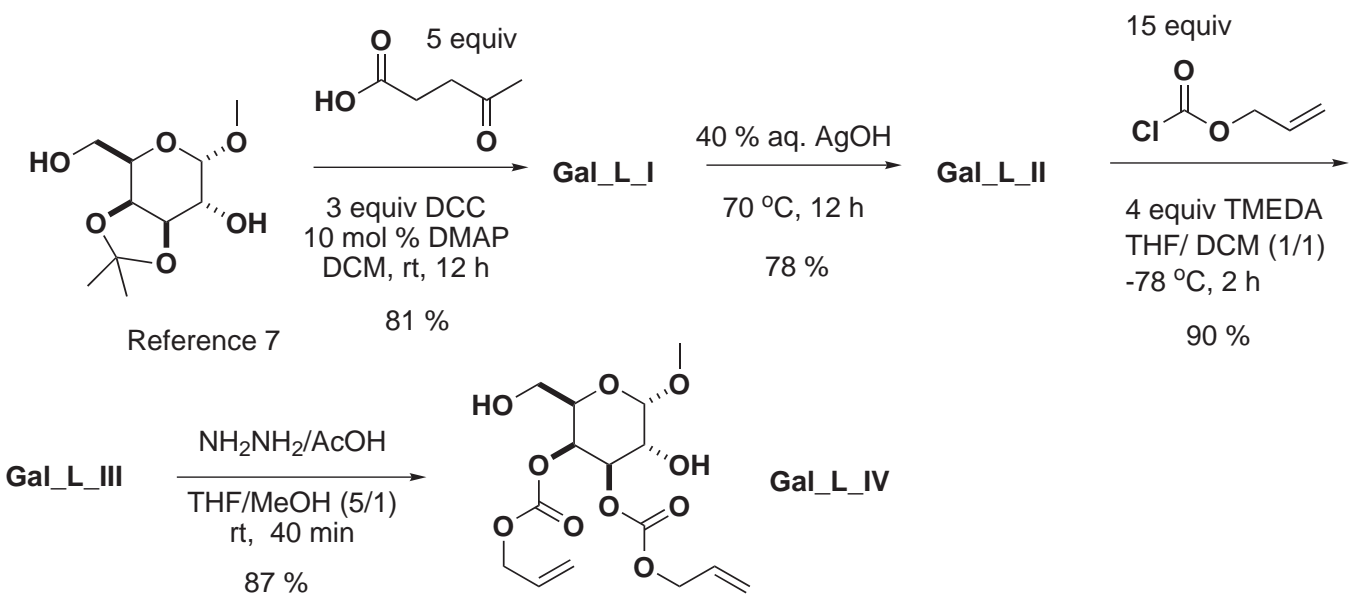

Scheme 3. Synthesis of 4,5-bis-protected carbohydrate derivatives 


\section{General procedure for the formation bis-allyloxycarbonates. ${ }^{8}$}

To a solution of diol $\left(\mathrm{x}_{1} \mathrm{mg}, \mathrm{x}_{2} \mathrm{mmol}\right)$ in TMEDA $\left(\mathrm{x}_{3} \mathrm{~mL}, \mathrm{x}_{4} \mathrm{mmol}\right)$ at $-78{ }^{\circ} \mathrm{C}$ was added allyloxycarbonyl chloride $\left(\mathrm{x}_{5} \mathrm{~mL}, \mathrm{x}_{6} \mathrm{mmol}\right)$ for $15 \mathrm{~min}$. The color of the resultant solution became pink immediately and then the following addition of DMAP $\left(\mathrm{x}_{7} \mathrm{mg}, \mathrm{x}_{8} \mathrm{mmol}\right)$ yielded a clear solution. The mixture was stirred at room temperature for another hour and quenched with $3 \mathrm{~N}$ aq. Hydrochloric acid. The resulting solution was extracted with EtOAc three times and the combined organic layer was washed with brine and dried over $\mathrm{Na}_{2} \mathrm{SO}_{4}$. After removal of the solvent, the residue was purified by flash chromatography on silica gel ( $\mathrm{x}_{9} \mathrm{~g}$, Hexanes/EtOAc: $\left.5 / 1\right)$ to afford product ( $\mathrm{x}_{10} \mathrm{mg}, \mathrm{x}_{11} \%$ yield).

\section{Glc_R_I}

$\mathbf{R}_{\mathbf{f}}=0.5(\mathrm{~A})$

H NMR $\left(500 \mathrm{MHz}, \mathrm{CDCl}_{3}\right): \delta 3.43(\mathrm{~s}, 3 \mathrm{H}), 3.67(\mathrm{t}, 1 \mathrm{H}, J=9.6), 3.78(\mathrm{t}, 1 \mathrm{H}, J=10.4), 3.94(\mathrm{ddd}, 1 \mathrm{H}$, $J=4.8, J=10.0, J=10.0), 4.32(\mathrm{dd}, 1 \mathrm{H}, J=4.8, J=10.0), 4.65(\mathrm{c}, 4 \mathrm{H}), 4.78(\mathrm{dd}, 1 \mathrm{H}, J=3.6, J=$ 10.0), 5.05 (d, $1 \mathrm{H}, J=3.6), 5.20$ (ddd, $1 \mathrm{H}, J=1.0, J=2.4, J=10.4), 5.30$ (c, 2H), 5.37 (ddd, $1 \mathrm{H}, J=1.5$, $J=2.7, J=17.2), 5.43$ (t, 1H, $J=9.6$ ), 5.51 (s, 1H), 5.89 (ddt, $1 \mathrm{H}, J=6.0, J=10.4, J=16.0$ ), 5.93 (ddt, $1 \mathrm{H}, J=6.0, J=10.4, J=17.2), 7.35(\mathrm{c}, 3 \mathrm{H}), 7.46(\mathrm{c}, 2 \mathrm{H})$

${ }^{13}$ C NMR (101 MHz, $\left.\mathrm{CDCl}_{3}\right): \delta$ 55.7, 62.4, $69.0(2), 69.2,73.3,74.8,79.3,97.6,101.8,119.1,119.3$, 126.4 (2), 128.4 (2), 129.3, 131.4, 131.5, 137.0, 154.2, 154.4

IR (NaCl): 2940 (w), 1758 (s), 1370 (m), 1274 (s), 1095 (m), 1050 (w), 988 (m) cm .

HRMS-ESI $(\mathrm{m} / z):[\mathrm{M}+\mathrm{H}]^{+}$calcd for $\mathrm{C}_{22} \mathrm{H}_{27} \mathrm{O}_{10}$ 451.1599; found, 451.1600.

$\left(\mathrm{x}_{1} \mathrm{x}_{2} \mathrm{x}_{3} \mathrm{x}_{4} \mathrm{x}_{5} \mathrm{x}_{6} \mathrm{x}_{7} \mathrm{x}_{8} \mathrm{x}_{9} \mathrm{x}_{10} \mathrm{x}_{11}: 278,1.0,0.60,4.0,1.6,15,12,0.1,200.401,89\right)$

\section{Gal_R_I}

$\mathbf{R}_{\mathbf{f}}=0.6(\mathrm{~A})$

1 H NMR (500 MHz, $\left.\mathrm{CDCl}_{3}\right): \delta 3.44(\mathrm{~s}, 3 \mathrm{H}), 3.76(\mathrm{br} \mathrm{s}, 1 \mathrm{H}), 3.80(\mathrm{~s}, 3 \mathrm{H}), 4.07(\mathrm{dd}, 1 \mathrm{H}, J=1.6, J=$ 12.8), 4.27 (dd, $1 \mathrm{H}, J=1.6, J=12.8$ ), 4.54 (d, $1 \mathrm{H}, J=3.2), 4.64$ (c, $4 \mathrm{H}), 5.16(\mathrm{~d}, 1 \mathrm{H}, J=3.2), 5.20$ (c, $7 \mathrm{H}) 5.50(\mathrm{~s}, 1 \mathrm{H}), 5.90(\mathrm{c}, 2 \mathrm{H}), 6.89(\mathrm{~d}, 2 \mathrm{H}, J=6.4), 7.43(\mathrm{~d}, 2 \mathrm{H}, J=8.4)$

${ }^{13}$ C NMR (101 MHz, $\left.\mathrm{CDCl}_{3}\right): \delta 55.5,55.9,62.3,69.0,69.1,69.2,71.6,72.2,73.8,97.8,101.0,113.7$, $119.2,119.3,127.8,130.2,131.5,154.3,154.4,160.3$

IR (NaCl): 2939 (m), 1750 (s), 1615 (m), 1519 (m), 1368 (m), 1277 (m), $1172(\mathrm{w}), 995$ (w) cm $\mathrm{cm}^{-1}$

HRMS-ESI $(\mathrm{m} / \mathrm{z}):[\mathrm{M}+\mathrm{H}]^{+}$calcd for $\mathrm{C}_{23} \mathrm{H}_{29} \mathrm{O}_{11}$ 481.1704; found, 481.1702.

$\left(\mathrm{x}_{1} \mathrm{x}_{2} \mathrm{x}_{3} \mathrm{x}_{4} \mathrm{x}_{5} \mathrm{x}_{6} \mathrm{x}_{7} \mathrm{x}_{8} \mathrm{x}_{9} \mathrm{x}_{10} \mathrm{x}_{11}: 440,1.5,0.90,6.0,2.4,23,18,0.15,350,540,75\right)$ 


\section{Man_R_I}

$\mathbf{R}_{\mathbf{f}}=0.6(\mathrm{~A})$

${ }^{1}$ H NMR (400 MHz, $\left.\mathrm{CDCl}_{3}\right): \delta 3.43(\mathrm{~s}, 3 \mathrm{H}), 3.88$ (app t, $\left.1 \mathrm{H}, J=10.0\right), 3.93(\mathrm{dt}, 1 \mathrm{H}, J=4.0, J=10.0)$, 4.12 (app t, $1 \mathrm{H}, J=10.0), 4.29$ (dd, $1 \mathrm{H}, J=4.0, J=9.6), 4.64$ (c, 4H), 4.80 (d, 1H, $J=1.2), 5.21-5.42$ (c, $6 \mathrm{H}), 5.57(\mathrm{~s}, 1 \mathrm{H}), 5.94(\mathrm{c}, 1 \mathrm{H}), 7.35(\mathrm{c}, 3 \mathrm{H}), 7.46(\mathrm{c}, 2 \mathrm{H})$

${ }^{13} \mathrm{C}$ NMR $\left(101 \mathrm{MHz}, \mathrm{CDCl}_{3}\right): \delta 55.5,63.9,68.8,69.1,69.4,72.4,73.5,76.1,99.5,102.2,119.1,119.7$, $126.5,128.5(2), 128.8,129.4,131.4,131.6,137.3,154.0,154.5$ IR (NaCl): 2932 (w), 1754 (s), 1276 (s), 1241 (m), 1095 (w), 1065 (w), $976(w) \mathrm{cm}^{-1}$.

HRMS-ESI $(\mathrm{m} / \mathrm{z}):[\mathrm{M}+\mathrm{H}]^{+}$calcd for $\mathrm{C}_{22} \mathrm{H}_{27} \mathrm{O}_{10} 451.1599$; found, 451.1604

$\left(\mathrm{x}_{1} \mathrm{x}_{2} \mathrm{x}_{3} \mathrm{x}_{4} \mathrm{x}_{5} \mathrm{x}_{6} \mathrm{x}_{7} \mathrm{x}_{8} \mathrm{x}_{9} \mathrm{x}_{10} \mathrm{x}_{11}: 308,0.11,0.66,4.4,1.8,17,14,0.11,200,426,86\right)$

\section{Glc_L_II}

$\mathbf{R}_{\mathbf{f}}=0.3(\mathrm{~B})$

${ }^{1}$ H NMR (500 MHz, $\left.\mathrm{C}_{6} \mathrm{D}_{6}\right): \delta 1.58(\mathrm{~s}, 3 \mathrm{H}), 1.62(\mathrm{~s}, 3 \mathrm{H}), 2.10-2.45(\mathrm{c}, 8 \mathrm{H}), 2.99(\mathrm{~s}, 3 \mathrm{H}), 3.75-3.84(\mathrm{~m}$, $1 \mathrm{H}), 4.13(\mathrm{dd}, 1 \mathrm{H}, J=2.5, J=12.5), 4.29$ (dd, $1 \mathrm{H}, J=4.5, J=12.5), 4.39-4.46$ (c, $4 \mathrm{H}), 4.92-4.96(\mathrm{c}, 2 \mathrm{H})$, $4.92(\mathrm{~d}, 1 \mathrm{H}, J=4.0), 5.08-5.14$ (c, 2H), 5.12 (dd, 1H, $J=4.0, J=10.0), 5.24$ (app t, $1 \mathrm{H}, J=9.8), 5.62-$ $5.71(\mathrm{c}, 2 \mathrm{H}), 5.81(\mathrm{t}, 1 \mathrm{H}, J=10.0)$

${ }^{13}$ C NMR (101 MHz, $\left.\mathrm{CDCl}_{3}\right): \delta 28.0,28.1,29.9,30.0,37.9,38.0,55.8,62.3,67.1,69.0,69.3,71.1,72.6$, $74.2,96.8,119.3,119.7,131.3,131.6,154.0,154.3,171.9,172.5,206.2,206.3$

IR (NaCl): 2955 (w), 1759 (s), 1719 (s), 1364 (m), 1238 (m), 1158 (m), 1038 (w), 968 (w), $784(w) \mathrm{cm}^{-1}$ HRMS-ESI $(\mathrm{m} / \mathrm{z}):[\mathrm{M}+\mathrm{H}]^{+}$calcd for $\mathrm{C}_{25} \mathrm{H}_{35} \mathrm{O}_{14}$ 559.2021; found, 559.2036.

$\left(\mathrm{x}_{1} \mathrm{x}_{2} \mathrm{x}_{3} \mathrm{x}_{4} \mathrm{x}_{5} \mathrm{x}_{6} \mathrm{x}_{7} \mathrm{x}_{8} \mathrm{x}_{9} \mathrm{x}_{10} \mathrm{x}_{11}: 395,1.0,0.6,4.0,1.6,15,12,0.1,200,520,93\right)$

\section{Gal_L_III}

$\mathbf{R}_{\mathbf{f}}=0.25(\mathrm{~B})$

H NMR (500 MHz, CDCl 3$): \delta 2.21(\mathrm{~s}, 3 \mathrm{H}), 2.22(\mathrm{~s}, 3 \mathrm{H}), 2.66(\mathrm{c}, 4 \mathrm{H}), 2.82(\mathrm{c}, 4 \mathrm{H}), 3.38(\mathrm{~s}, 3 \mathrm{H}), 3.69$ (app t, $1 \mathrm{H}, J=9.5), 3.72(\mathrm{dd}, 1 \mathrm{H}, J=6.8, J=11.7), 4.00(\mathrm{~d}, 1 \mathrm{H}, J=6.8), 4.04(\mathrm{dd}, 1 \mathrm{H}, J=3.4, J=10.3)$, 4.62 (brs, 4H), 4.86 (d, 1H, $J=3.4), 4.98$ (dd, 1H, $J=3.4, J=10.3$ ), 5.27 (c, 2H), 5.29 (d, 1H, $J=3.4$ ), 5.35 (c, 2H) $5.92(\mathrm{c}, 2 \mathrm{H})$

${ }^{13}$ C NMR (101 MHz, $\left.\mathrm{CDCl}_{3}\right): \delta 28.0,28.0,29.9,30.0,37.9,38.0,55.8,62.3,67.1,69.0,69.3,71.1,72.6$, $74.2,96.8,119.0,119.3,131.3,131.6,154.1,154.4,171.9,172.5,206.4,206.6$

IR (NaCl): 2919 (m), 1737 (s), 1716 (s), 1409 (w), 1362 (m), 1135 (m), 1063 (w), 969 (w) cm . HRMS-ESI $(\mathrm{m} / \mathrm{z}):[\mathrm{M}+\mathrm{H}]^{+}$calcd for $\mathrm{C}_{25} \mathrm{H}_{35} \mathrm{O}_{14}$ 559.2021; found, 559.2030.

$\left(\mathrm{x}_{1} \mathrm{x}_{2} \mathrm{x}_{3} \mathrm{x}_{4} \mathrm{x}_{5} \mathrm{x}_{6} \mathrm{x}_{7} \mathrm{x}_{8} \mathrm{x}_{9} \mathrm{x}_{10} \mathrm{x}_{11}: 198,0.5,0.3,2.0,0.8,7.5,6,0.05,200,248,90\right)$ 


\section{Man_L_III}

$\mathbf{R}_{\mathbf{f}}=0.3(\mathrm{~B})$

${ }^{1}$ H NMR $\left(500 \mathrm{MHz}, \mathrm{CDCl}_{3}\right): \delta 2.20(\mathrm{~s}, 6 \mathrm{H}), 2.62-2.83(\mathrm{c}, 8 \mathrm{H}), 3.40(\mathrm{~s}, 3 \mathrm{H}), 3.64(\mathrm{~m}, 1 \mathrm{H}), 4.22(\mathrm{dd}, 1 \mathrm{H}$, $J=3.3, J=15.0), 4.32(\mathrm{dd}, 1 \mathrm{H}, J=7.2, J=15.0), 4.62(\mathrm{~m}, 4 \mathrm{H}), 4.72($ app s, 1H), 5.10 (app t, $1 \mathrm{H}, J=$ $12.3), 5.16(\mathrm{dd}, 1 \mathrm{H}, J=4.2, J=12.3), 5.24-5.37$ (c, $5 \mathrm{H}), 5.86-5.96(\mathrm{c}, 2 \mathrm{H})$

${ }^{13}$ C NMR (101 MHz, $\left.\mathrm{CDCl}_{3}\right): \delta 28.0,28.1,30.0,30.0,38.0,38.1,55.6,62.8,68.2,69.1,69.2,69.5,70.4$, 73.0, 98.5, 119.1, 119.3, 131.3, 131.5, 153.8, 154.2, 171.9, 172.5, 206.3, 206.5

IR (NaCl): 2956 (m), 1755 (s), 1752 (s), 1649 (w), 1365 (m), 1277 (s), 1134 (m), 933 (m), 784 (w) cm .

HRMS-ESI $(\mathrm{m} / \mathrm{z}):[\mathrm{M}+\mathrm{H}]^{+}$calcd for $\mathrm{C}_{25} \mathrm{H}_{35} \mathrm{O}_{14}$ 559.2021; found, 559.2029.

$\left(\mathrm{x}_{1} \mathrm{x}_{2} \mathrm{x}_{3} \mathrm{x}_{4} \mathrm{x}_{5} \mathrm{x}_{6} \mathrm{x}_{7} \mathrm{x}_{8} \mathrm{x}_{9} \mathrm{x}_{10} \mathrm{x}_{11}: 395,1.0,0.6,4.0,1.6,15,12,0.1,200,492,88\right)$ 


\section{General procedure for the preparation of bis-levulinate}

To a mixture of substrate diol $\left(\mathrm{x}_{1} \mathrm{mg}, \mathrm{x}_{2} \mathrm{mmol}\right)$ and levulinic acid $\left(\mathrm{x}_{3} \mathrm{mg}, \mathrm{x}_{4} \mathrm{mmol}\right)$ in $\mathrm{CH}_{2} \mathrm{Cl}_{2}\left(\mathrm{x}_{5}\right.$ $\mathrm{mL}$ ) was added 1,3-dicyclcohexylcarbodiimide ( $\left.\mathrm{x}_{6} \mathrm{mg}, \mathrm{x}_{7} \mathrm{mmol}\right)$ DMAP ( $\left.\mathrm{x}_{8} \mathrm{mg}, \mathrm{x}_{9} \mathrm{mmol}\right)$. The reaction mixture was stirred for $12 \mathrm{~h}$ at room temperature. The mixture was diluted with Hexane/ diethyl ether $(1 / 1)$ and then filtered to get rid of white solid materials. The filtrate was diluted with $\mathrm{CH}_{2} \mathrm{Cl}_{2}$ and washed with saturated $\mathrm{NaHCO}_{3}$ and brine. The organic layer was dried over $\mathrm{Na}_{2} \mathrm{SO}_{4}$. After removal of the solvent, the residue was purified by flash chromatography on silica gel ( $\mathrm{x}_{10} \mathrm{~g}$, Hexanes/EtOAc: $\left.\mathrm{x}_{11}\right)$ to give product ( $\mathrm{x}_{12} \mathrm{mg}, \mathrm{x}_{13} \%$ yield).

Gal_L_I

$\mathbf{R}_{\mathbf{f}}=0.3(\mathrm{C})$

${ }^{1}$ H NMR (500 MHz, CDCl 3$): \delta 1.27$ (s, 3H), $1.28(\mathrm{~s}, 3 \mathrm{H}), 2.18(\mathrm{~s}, 3 \mathrm{H}), 2.19(\mathrm{~s}, 3 \mathrm{H}), 2.57-2.76(\mathrm{c}, 8 \mathrm{H})$, 3.39 (s, 3H), 4.16 (ddd, $1 \mathrm{H}, J=2.5, J=4.0, J=7.8), 4.21$ (dd, $1 \mathrm{H}, J=2.5, J=5.0), 4.32$ (dd, $1 \mathrm{H}, J=7.8$, $J=12.0), 4.32(\mathrm{dd}, 1 \mathrm{H}, J=5.0, J=8.0), 4.39(\mathrm{dd}, 1 \mathrm{H}, J=4.0, J=12.0), 4.82(\mathrm{~d}, 1 \mathrm{H}, J=4.0), 4.90(\mathrm{dd}$, $1 \mathrm{H}, J=4.0, J=7.8)$

${ }^{13}$ C NMR (101 MHz, $\left.\mathrm{CDCl}_{3}\right): \delta 17.8,17.9,28.0,28.1,30.1,30.1,38.0,38.1,55.4,62.6,66.5,67.0,67.4$, 71.0, 97.3, 100.0, 172.3, 172.8, 206.4, 206.5

IR (NaCl): 2975 (w), 1738 (s), 1718 (s), 1408 (m), 1362 (m), $1311(\mathrm{~m}), 1077$ (w) cm .

HRMS-ESI $(\mathrm{m} / \mathrm{z}):[\mathrm{M}+\mathrm{H}]^{+}$calcd for $\mathrm{C}_{20} \mathrm{H}_{31} \mathrm{O}_{10}, 431.1917$; found, 431.1926.

$\left(\mathrm{x}_{1} \mathrm{x}_{2} \mathrm{x}_{3} \mathrm{x}_{4} \mathrm{x}_{5} \mathrm{x}_{6} \mathrm{x}_{7} \mathrm{x}_{8} \mathrm{x}_{9} \mathrm{x}_{10} \mathrm{x}_{11} \mathrm{x}_{12} \mathrm{x}_{13}: 232,1.0,0.5,5.0,15,620,3.0,12,0.1,300,4 / 1,183,88\right)$

\section{Man_L_I}

$\mathbf{R}_{\mathbf{f}}=0.4(\mathrm{C})$

${ }^{1}$ H NMR (500 MHz, CDCl 3$): \delta 2.21(\mathrm{~s}, 3 \mathrm{H}), 2.22(\mathrm{~s}, 3 \mathrm{H}), 2.66(\mathrm{c}, 4 \mathrm{H}), 2.82(\mathrm{c}, 4 \mathrm{H}), 3.38(\mathrm{~s}, 3 \mathrm{H}), 3.69$ (app t, $1 \mathrm{H}, J=9.5), 3.72(\mathrm{dd}, 1 \mathrm{H}, J=6.8, J=11.7), 4.00(\mathrm{~d}, 1 \mathrm{H}, J=6.8), 4.04$ (dd, $1 \mathrm{H}, J=3.4, J=10.3)$, 4.62 (brs, $4 \mathrm{H}), 4.86$ (d, 1H, $J=3.4$ ), 4.98 (dd, 1H, $J=3.4, J=10.3), 5.27$ (c, 2H), 5.29 (d, 1H, $J=3.4$ ), $5.35(\mathrm{c}, 2 \mathrm{H}) 5.92(\mathrm{c}, 2 \mathrm{H})$

${ }^{13}$ C NMR (101 MHz, $\left.\mathrm{CDCl}_{3}\right)$ : $\delta 17.9,18.0,28.1,28.6,30.1,30.2,38.1,38.4,48.2,48.4,55.3,63.1,63.9$, $66.3,68.8,70.7,99.2,100.2,100.5,172.3,172.9,206.6,206.7$

IR (NaCl): 2970 (w), 1753 (s), 1720 (s), 1366 (m), 1130 (m), 1049 (w), 933 (w), 850 (w) cm .

HRMS-ESI $(\mathrm{m} / \mathrm{z})$ : $\left[\mathrm{M}+\mathrm{NH}_{4}\right]^{+}$calcd for $\mathrm{C}_{23} \mathrm{H}_{40} \mathrm{NO}_{12}$ 522.2545; found, 522.2550 .

$\left(\mathrm{x}_{1} \mathrm{x}_{2} \mathrm{x}_{3} \mathrm{x}_{4} \mathrm{x}_{5} \mathrm{x}_{6} \mathrm{x}_{7} \mathrm{x}_{8} \mathrm{x}_{9} \mathrm{x}_{10} \mathrm{x}_{11} \mathrm{x}_{12} \mathrm{x}_{13}: 158,0.5,0.25,2.510,310,1.5,6,0.05,200,3 / 1,215,83\right)$ 


\section{Glc_L_II}

$\mathbf{R}_{\mathbf{f}}=0.2(\mathrm{C})$

${ }^{1}$ H NMR $\left(500 \mathrm{MHz}, \mathrm{CDCl}_{3}\right): \delta 2.21(\mathrm{~s}, 3 \mathrm{H}), 2.22(\mathrm{~s}, 3 \mathrm{H}), 2.62-2.67(\mathrm{c}, 4 \mathrm{H}), 2.79-2.84(\mathrm{c}, 4 \mathrm{H}), 3.41(\mathrm{~s}$, $3 \mathrm{H}), 3.50$ (app t, $1 \mathrm{H}, J=9.5), 3.79(\mathrm{~m}, 1 \mathrm{H}), 4.00$ (app t, $1 \mathrm{H}, J=9.7), 4.28$ (dd, $1 \mathrm{H}, J=2.4, J=12.5)$, $4.54(\mathrm{dd}, 1 \mathrm{H}, J=5.0, J=12.5), 4.78(\mathrm{dd}, 1 \mathrm{H}, J=3.4, J=9.7), 4.86(\mathrm{~d}, 1 \mathrm{H}, J=3.4)$

${ }^{13} \mathbf{C}$ NMR $\left(101 \mathrm{MHz}, \mathrm{CDCl}_{3}\right): \delta 28.1,28.2,30.0,30.1,38.1,38.2,55.4,63.6,69.5,70.5,71.5,73.3,92.3$, $172.7,173.3,207.4,207.7$

IR (NaCl): 3084 (w), 2973 (m), 2950 (m), 2903 (s), 1480 (m), 1444 (m), 1419 (m), 1381 (s), 1369 (m), $1271(\mathrm{~m}), 1032(\mathrm{~m}), 941(\mathrm{~m}), 821(\mathrm{~m}) \mathrm{cm}^{-1}$.

HRMS-ESI $(\mathrm{m} / \mathrm{z}):[\mathrm{M}+\mathrm{Na}]^{+}$calcd for $\mathrm{C}_{17} \mathrm{H}_{26} \mathrm{NaO}_{10} 413.1424$; found, 413.1430 . 


\section{General procedure for the removal of Ley's protection group. ${ }^{6}$}

To a solution of dilevulinates (Man_L_I, $314 \mathrm{mg}, 0.6 \mathrm{mmol})$ in $\mathrm{CH}_{2} \mathrm{Cl}_{2}(10 \mathrm{~mL}$ ) was added $90 \%$ aqueous trifluoroacetic acid $(0.33 \mathrm{~mL}, 1.8 \mathrm{mmol})$ for $5 \mathrm{~min}$ at $0{ }^{\circ} \mathrm{C}$. Subsequent addition of triethylsilane $(0.3 \mathrm{~mL}, 2.0 \mathrm{mmol})$ made the resultant reaction mixture clearer. The mixture was stirred at room temperature for $20 \mathrm{~min}$ and then dried under high vacuum $(30 \mathrm{mmHg})$ for an hour. The resultant mixture was diluted with EtOAc and washed with saturated $\mathrm{NaHCO}_{3}$ and brine, and dried over $\mathrm{Na}_{2} \mathrm{SO}_{4}$. The dried organic layer was concentrated under reduced pressure, and the residue was purified by flash chromatography on a silica gel column (200 g, Hexanes/EtOAC: 1/1) to give product $(238 \mathrm{mg}, 97 \%$ yields) as a thick oil;

\section{Man_L_II}

$\mathbf{R}_{\mathbf{f}}=0.1(\mathrm{~B}), 0.2(\mathrm{C})$

${ }^{1}$ H NMR $\left(500 \mathrm{MHz}, \mathrm{CDCl}_{3}\right): \delta 2.20(\mathrm{~s}, 3 \mathrm{H}), 2.20(\mathrm{~s}, 3 \mathrm{H}), 2.56-2.72(\mathrm{c}, 4 \mathrm{H}), 2.74-2.90(\mathrm{c}, 4 \mathrm{H}), 3.38(\mathrm{~s}$, $3 \mathrm{H}), 3.66$ (app t, $1 \mathrm{H}, J=12.4), 3.75(\mathrm{ddd}, 1 \mathrm{H}, J=2.3, J=5.5, J=12), 3.96(\mathrm{dd}, 1 \mathrm{H}, J=4.5, J=10.5)$, $4.27(\mathrm{dd}, 1 \mathrm{H}, J=2.3, J=15.0), 4.56(\mathrm{dd}, 1 \mathrm{H}, J=5.5, J=15.0), 4.68(\mathrm{~d}, 1 \mathrm{H}, J=1.8), 5.16(\mathrm{dd}, 1 \mathrm{H}, J=$ $1.8, J=3.5)$

${ }^{13}$ C NMR (101 MHz, $\left.\mathrm{CDCl}_{3}\right): \delta 28.0,28.2,30.0,30.0,38.1,38.3,55.4,63.9,68.0,70.2,70.5,72.2,98.9$, $172.8,173.7,208.2,208.5$

IR (NaCl): 3608 (m), 3446 (s), $2920(\mathrm{~m}), 1653(\mathrm{~s}), 1646(\mathrm{~s}), 1616(\mathrm{~m}), 668(\mathrm{~m}) \mathrm{cm}^{-1}$.

HRMS-ESI $(\mathrm{m} / \mathrm{z}):\left[\mathrm{M}+\mathrm{NH}_{4}\right]^{+}$calcd for $\mathrm{C}_{17} \mathrm{H}_{30} \mathrm{NO}_{10} 408.1864$; found, 408.1 . 


\section{General procedure for the removal of the benzylideneacetal protection groups. ${ }^{9}$}

The mixture of starting material $\left(\mathrm{x}_{1} \mathrm{mg}, \mathrm{x}_{2} \mathrm{mmol}\right)$ in $40 \%$ aqueous acetic acid $\left(\mathrm{x}_{3} \mathrm{~mL}\right)$ was heated at $77{ }^{\circ} \mathrm{C}$ for 15 hours. The solution was diluted with ethanol and evaporated under reduced pressure at room temperature. The resultant solution was diluted with $\mathrm{CH}_{2} \mathrm{Cl}_{2}$ and washed with saturated $\mathrm{NaHCO}_{3}$ and brine, and dried over $\mathrm{Na}_{2} \mathrm{SO}_{4}$. The dried organic layer was concentrated under reduced pressure, and the residue was purified by chromatography on a silica gel column $\left(\mathrm{x}_{4} \mathrm{~g}\right.$, Hexanes/EtOAC: $\left.\mathrm{x}_{5}\right)$ to give corresponding product $\left(\mathrm{x}_{6} \mathrm{mg}, \mathrm{x}_{7} \%\right.$ yield).

\section{Glc_R_II}

$\mathbf{R}_{\mathbf{f}}=0.4(\mathrm{D})$

${ }^{1}$ H NMR (500 MHz, $\left.\mathrm{CDCl}_{3}\right): \delta 3.42(\mathrm{~s}, 3 \mathrm{H}), 3.72(\mathrm{dt}, 1 \mathrm{H}, J=4.0, J=9.8), 3.80$ (app t, $1 \mathrm{H}, J=9.3$ ), $3.86(\mathrm{dd}, 1 \mathrm{H}, J=4.0, J=12.0), 3.90(\mathrm{dt}, 1 \mathrm{H}, J=4.0, J=12), 4.64(\mathrm{c}, 4 \mathrm{H}), 4.69$ (dd, 1H, $J=4.0, J=$ 9.0), 5.01 (d, 1H, $J=4.0), 5.17$ (app t, $1 \mathrm{H}, J=9.5), 5.28$ (c, 2H), 5.36 (c, 2H), 5.93 (c, 2H)

${ }^{13} \mathbf{C}$ NMR $\left(101 \mathrm{MHz}, \mathrm{CDCl}_{3}\right): \delta 55.9,61.0,69.1,69.4,69.8,71.3,72.1,77.2,99.5,119.1,119.5,131.2$, $131.5,155.0,155.2$

IR (NaCl): 3502 (br w), 2941 (m), 1734 (s), 1373 (w), 1266 (m), 1030 (w), 947 (w) cm . $^{-1}$

HRMS-ESI $(\mathrm{m} / \mathrm{z}):[\mathrm{M}+\mathrm{H}]^{+}$calcd for $\mathrm{C}_{15} \mathrm{H}_{23} \mathrm{O}_{10} 363.1291$; found, 363.1292

$\left(\mathrm{x}_{1} \mathrm{x}_{2} \mathrm{x}_{3} \mathrm{x}_{4} \mathrm{x}_{5} \mathrm{x}_{6}: 90,0.2,10,100,2 / 1,62,85\right)$

\section{Gal_R_II}

$\mathbf{R}_{\mathbf{f}}=0.4(\mathrm{D})$

H NMR (500 MHz, $\mathrm{CDCl}_{3}$ ): $\delta 2.63$ (brs, 1H), 3.24 (brs, 1H), 3.42 (s, 3H), 3.91 (c, 3H), 4.36 (brs, 1H), $4.64(\mathrm{c}, 4 \mathrm{H}), 5.09$ (d 1H, $J=3.2), 5.12(\mathrm{dd}, 1 \mathrm{H}, J=2.3, J=10.0), 5.15(\mathrm{dd}, 1 \mathrm{H}, J=3.2, J=10.0), 5.28$ (c, 2H), $5.36(\mathrm{c}, 2 \mathrm{H}), 5.93(\mathrm{c}, 2 \mathrm{H})$

${ }^{13}$ C NMR $\left(101 \mathrm{MHz}, \mathrm{CDCl}_{3}\right): \delta 55.7,63.1,69.0,69.1,69.20,69.23,71.7,74.1,97.3,119.3,119.6$, $131.36,131.43,154.1,154.5$

IR (NaCl): 3502 (br w), 2945 (m), 1750 (s), 1373 (w), 1280 (s), 1050 (w), 947 (w) cm cm $^{-1}$

HRMS-ESI $(\mathrm{m} / \mathrm{z}):[\mathrm{M}+\mathrm{H}]^{+}$calcd for $\mathrm{C}_{15} \mathrm{H}_{23} \mathrm{O}_{10}$ 363.1291; found, 363.1297.

$\left(\mathrm{x}_{1} \mathrm{x}_{2} \mathrm{x}_{3} \mathrm{x}_{4} \mathrm{x}_{5} \mathrm{x}_{6}: 225,0.5,20,200,3 / 2,160,88\right)$ 


\section{Man_R_II}

$\mathbf{R}_{\mathbf{f}}=0.3(\mathrm{D})$

${ }^{1}$ H NMR $\left(500 \mathrm{MHz}, \mathrm{CDCl}_{3}\right): \delta 3.41(\mathrm{~s}, 3 \mathrm{H}), 3.72(\mathrm{dt}, 1 \mathrm{H}(\mathrm{C} 5 \mathrm{HO}), J=3.5, J=10.0), 3.89(\mathrm{c}, 2 \mathrm{H}), 4.08$ (ddd, 1H, $J=4.7, J=9.5, J=10.0$ ), 4.63 (d, 2H, $J=5.5$ ), 4.66 (d, 2H, $J=5.5$ ), 4.80 (br s, 1H), 5.02 (dd, $1 \mathrm{H}, J=3.5, J=9.5$ ), 5.17 (dd, $1 \mathrm{H}, J=2.0, J=3.5$ ), 5.29 (d, 2H, $J=10.5), 5.37$ (c, $2 \mathrm{H}, J=, J=), 5.94$ $(\mathrm{m}, 2 \mathrm{H}, J=10.0)$

${ }^{13}$ C NMR (101 MHz, $\left.\mathrm{CDCl}_{3}\right): \delta 55.5,62.4,66.0,69.3$ (2C), 72.2, 73.1, 76.1, 98.7, 119.5, 119.6, 131.3, $131.4,154.5,154.6$

IR (NaCl): 3502 (br w), 2934 (m), 1753 (s), 1371 (m), 1278 (s), 1138 (w), $1071(\mathrm{~m}), 952$ (w) $\mathrm{cm}^{-1}$. HRMS-ESI $(\mathrm{m} / z):[\mathrm{M}+\mathrm{H}]^{+}$calcd for $\mathrm{C}_{15} \mathrm{H}_{23} \mathrm{O} 10$ 363.1291; found, 363.1283 .

$\left(\mathrm{x}_{1} \mathrm{x}_{2} \mathrm{x}_{3} \mathrm{x}_{4} \mathrm{x}_{5} \mathrm{x}_{6}: 144,0.3,10,200,2 / 1,99,91\right)$

\section{Gal_L_II}

$\mathbf{R}_{\mathbf{f}}=0.3(\mathrm{E})$

${ }^{1}$ H NMR (500 MHz, CDCl 3$): \delta 2.21(\mathrm{~s}, 3 \mathrm{H}), 2.22(\mathrm{~s}, 3 \mathrm{H}), 2.66(\mathrm{c}, 4 \mathrm{H}), 2.82(\mathrm{c}, 4 \mathrm{H}), 3.38$ (s, 3H), 3.69 (app t, $1 \mathrm{H}, J=9.5), 3.72(\mathrm{dd}, 1 \mathrm{H}, J=6.8, J=11.7), 4.00(\mathrm{~d}, 1 \mathrm{H}, J=6.8), 4.04(\mathrm{dd}, 1 \mathrm{H}, J=3.4, J=10.3)$, 4.62 (brs, 4H), 4.86 (d, 1H, $J=3.4$ ), 4.98 (dd, 1H, $J=3.4, J=10.3$ ), 5.27 (c, 2H), 5.29 (d, 1H, $J=3.4$ ), $5.35(\mathrm{c}, 2 \mathrm{H}) 5.92(\mathrm{c}, 2 \mathrm{H})$

${ }^{13} \mathrm{C}$ NMR $\left(101 \mathrm{MHz}, \mathrm{CDCl}_{3}\right): \delta 28.0,28.3,30.0,30.0,38.0,38.1,55.5,63.6,67.8,68.1,69.5,71.7,97.5$, $173.0,173.2,207.9,208.2$

IR (NaCl): 3435(s), 2919(m), 1737(s), 1716(s), 1409(w), 1362(m), 1135(m), 1063(w), 969(w) cm .

HRMS-ESI $(m / z)$ : $[\mathrm{M}+\mathrm{Na}]^{+}$calcd for $\mathrm{C}_{17} \mathrm{H}_{26} \mathrm{NaO}_{10} 413.1424$; found, 413.1430

$\left(\mathrm{x}_{1} \mathrm{x}_{2} \mathrm{x}_{3} \mathrm{x}_{4} \mathrm{x}_{5} \mathrm{x}_{6}: 230,0.5,20,200,2 / 1,162,78\right)$ 


\section{General procedure for the deprotection of bis-levulinates to diol. ${ }^{10}$}

To a stirred solution of levulinates $\left(\mathrm{x}_{1} \mathrm{mg}, \mathrm{x}_{2} \mathrm{mmol}\right)$ in THF-MeOH $\left(10: 1 \mathrm{v} / \mathrm{v}, \mathrm{x}_{3} \mathrm{~mL}\right), 1 \mathrm{M}$ hydrazine-acetic acid (1: $2.5 \mathrm{v} / \mathrm{v})$ in THF-MeOH $\left(5: 1 \mathrm{v} / \mathrm{v}, \mathrm{x}_{4} \mathrm{~mL}\right)$ was added. The reaction mixture was stirred for $40 \mathrm{~min}$. The solvent was then removed and the residue was diluted with EtOAc $(20 \mathrm{~mL})$. The organic layer was washed with saturated $\mathrm{NaHCO}_{3}$ and brine, and dried over $\mathrm{Na}_{2} \mathrm{SO}_{4}$. The dried organic layer was concentrated under reduced pressure, and the residue was purified by flash chromatography on a silica gel column ( $\mathrm{x}_{5} \mathrm{~g}$, Hexanes/EtOAC: $\left.\mathrm{x}_{6}\right)$ to give corresponding diol product $\left(\mathrm{x}_{7} \mathrm{mg}, \mathrm{x}_{8} \%\right.$ yields$)$.

\section{Gle_L_III}

$\mathbf{R}_{\mathbf{f}}=0.3(\mathrm{D})$

${ }^{1}$ H NMR (500 MHz, CDCl3): $\delta 3.46$ (s, 3H), 3.75 (c, 4H), 4.65 (c, 4H), 4.84 (app t, 1H, J= 9.8), 4.84 (d, $1 \mathrm{H}, J=3.4), 4.64$ (c, 4H), 5.13 (app t, 1H, $J=9.8), 5.30$ (c, 4H), 5.93 (c, 2H)

${ }^{13}$ C NMR $\left(101 \mathrm{MHz}, \mathrm{CDCl}_{3}\right): \delta 55.6,62.0,69.2,69.3,71.3,74.2,77.2,96.9,119.3,119.4,131.4(2 \mathrm{C})$ $154.4,155.6$

IR (NaCl): 3084 (w), 2973 (m), 2950 (m), 2903 (s), 1480 (m), 1444 (m), 1419 (m), 1381 (s), 1369 (m), $1271(\mathrm{~m}), 1032(\mathrm{~m}), 941(\mathrm{~m}), 821(\mathrm{~m}) \mathrm{cm}^{-1}$.

HRMS-ESI $(\mathrm{m} / \mathrm{z}):[\mathrm{M}+\mathrm{H}]^{+}$calcd for $\mathrm{C}_{15} \mathrm{H}_{23} \mathrm{O}_{10} 363.1291$; found, 363.1287.

$\left(\mathrm{x}_{1} \mathrm{x}_{2} \mathrm{x}_{3} \mathrm{x}_{4} \mathrm{x}_{5} \mathrm{x}_{6} \mathrm{x}_{7} \mathrm{x}_{8}: 413,1.0,10,7.0,300,1 / 1,320,88\right)$

\section{Gal_L_IV}

$\mathbf{R}_{\mathbf{f}}=0.3$ (D)

${ }^{1}$ H NMR (500 MHz, $\left.\mathrm{CDCl}_{3}\right): \delta 3.43(\mathrm{~s}, 3 \mathrm{H}), 3.59(\mathrm{dd}, 1 \mathrm{H}, J=6.3, J=11.7), 3.72(\mathrm{dd}, 1 \mathrm{H}, J=6.8, J=$ 11.7), 4.00 (d, 1H, $J=6.8), 4.04$ (dd, $1 \mathrm{H}, J=3.4, J=10.3$ ), 4.62 (brs, $4 \mathrm{H}), 4.86$ (d, 1H, $J=3.4), 4.98$ $(\mathrm{dd}, 1 \mathrm{H}, J=3.4, J=10.3), 5.27(\mathrm{c}, 2 \mathrm{H}), 5.29(\mathrm{~d}, 1 \mathrm{H}, J=3.4), 5.35(\mathrm{c}, 2 \mathrm{H}) 5.92(\mathrm{c}, 2 \mathrm{H})$

${ }^{13}$ C NMR $\left(101 \mathrm{MHz}, \mathrm{CDCl}_{3}\right): \delta 55.9,61.0,69.1,69.4,69.971 .3,72.1,77.2,99.5,119.1,119.6,131.2$, $131.5,155.0,155.3$

IR (NaCl): 3084 (w), 2973 (m), 2950 (m), 2903 (s), 1480 (m), 1444 (m), 1419 (m), 1381 (s), 1369 (m), $1271(\mathrm{~m}), 1032(\mathrm{~m}), 941(\mathrm{~m}), 821(\mathrm{~m}) \mathrm{cm}^{-1}$.

HRMS-ESI (m/z): $[\mathrm{M}++\mathrm{H}]^{+}$calcd for $\mathrm{C}_{15} \mathrm{H}_{23} \mathrm{O}_{10} 363.1291$; found, 363.1290 .

$\left(\mathrm{x}_{1} \mathrm{x}_{2} \mathrm{x}_{3} \mathrm{x}_{4} \mathrm{x}_{5} \mathrm{x}_{6} \mathrm{x}_{7} \mathrm{x}_{8}: 215,0.5,10,5,200,3 / 2,158,87\right)$ 
Man_L_IV

$\mathbf{R}_{\mathbf{f}}=0.3(\mathrm{D})$

${ }^{1}$ H NMR $\left(400 \mathrm{MHz}, \mathrm{CDCl}_{3}\right): \delta 3.41(\mathrm{~s}, 3 \mathrm{H}), 3.71(\mathrm{dd}, 1 \mathrm{H}, J=4.2, J=12.1), 3.78(\mathrm{c}, 2 \mathrm{H}), 4.17$ (br s, $1 \mathrm{H}), 4.64(\mathrm{~s}, 2 \mathrm{H}), 4.65(\mathrm{~s}, 2 \mathrm{H}), 4.80(\mathrm{~d}, 1 \mathrm{H}, J=1.6), 5.15(\mathrm{dd}, 1 \mathrm{H}, J=3.0, J=10.0), 5.20(\mathrm{t}, 1 \mathrm{H}, J=$ 10.0), $5.28(\mathrm{c}, 2 \mathrm{H}) 5.36(\mathrm{c}, 2 \mathrm{H}) 5.92(\mathrm{c}, 2 \mathrm{H})$

${ }^{13}$ C NMR (101 MHz, $\left.\mathrm{CDCl}_{3}\right): \delta 55.5\left(\mathrm{CH}_{3}\right), 61,2\left(\mathrm{CH}_{2} \mathrm{OH}\right), 69.1\left(\mathrm{OCH}_{2}\right), 69.2(\mathbf{C H}), 69.3\left(\mathrm{OCH}_{2}\right), 70.3$, $(\mathbf{C H}) 70.4(\mathrm{CH}), \quad 75.6(\mathrm{CH}), \quad 100.7(\mathrm{OCHOMe}), \quad 119.3\left(\mathrm{CHCH}_{2}\right), \quad 119.4\left(\mathrm{CHCH}_{2}\right), \quad 131.3\left(\mathrm{CH}_{2} 2 \mathrm{CH}\right)$, 131.5 $\left(\mathrm{CH}_{2} \mathbf{C H}\right), 154.1(\mathrm{CO}), 155.0(\mathbf{C O})$.

IR (NaCl): 3502 (br w), 2941 (m), 1751 (s), 1369 (w), 1276 (m), 1129 (w), 1060 (w), 990 (w) $\mathrm{cm}^{-1}$. HRMS-ESI $(\mathrm{m} / z):[\mathrm{M}+\mathrm{H}]^{+}$calcd for $\mathrm{C}_{15} \mathrm{H}_{23} \mathrm{O} 10$ 363.1291; found, 363.1287 .

$\left(\mathrm{x}_{1} \mathrm{x}_{2} \mathrm{x}_{3} \mathrm{x}_{4} \mathrm{x}_{5} \mathrm{x}_{6} \mathrm{x}_{7} \mathrm{x}_{8}: 165,0.4,10,4,200,2 / 1,131,90\right)$ 


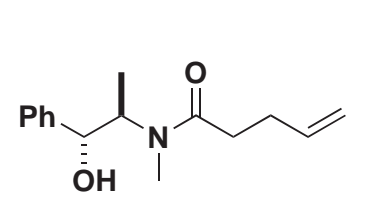

Reference 11<smiles>C=CCCC(=O)N1C(=O)OCC1Br</smiles>

Reference 12

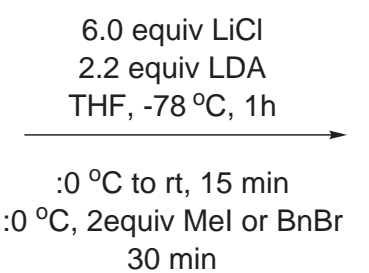

$>93 \%$

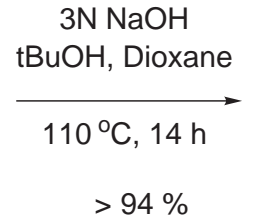<smiles>C=CCC(C)C(=O)O</smiles>

Me_alpha<smiles>C=CCC(Cc1ccccc1)C(=O)O</smiles>

Bn_alpha
1.8 equiv

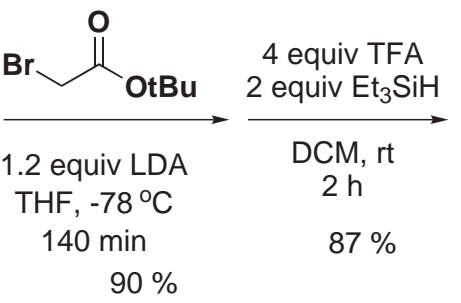

3 equiv

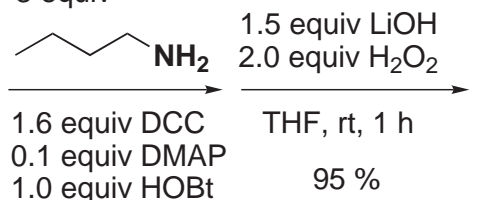

1.0 equiv $\mathrm{HOBt}$

DCM, rt, 3 h

$82 \%$

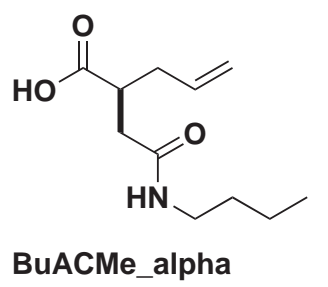

BuACMe_alpha<smiles>O=C(CCc1nc2ccccc2o1)N1C(=O)OCC1Cc1ccccc1</smiles>

Reference 13

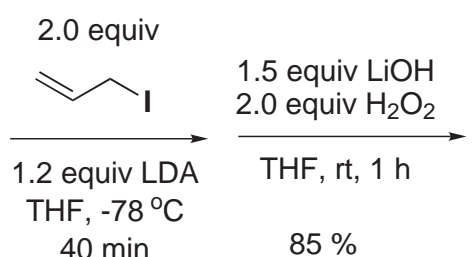

$92 \%$<smiles>C=CCC(Cc1nc2ccccc2o1)C(=O)O</smiles>

BoxMe_alpha

Scheme 4. Stereoselective synthesis of $\alpha$-substituted 4-pentenoic acid

\section{(R)-2-methylpent-4-enoic acid}

'H NMR (500 MHz, $\left.\mathrm{CDCl}_{3}\right): \delta 1.19(\mathrm{~d}, 3 \mathrm{H}, J=6.5), 3.59(\mathrm{dd}, 1 \mathrm{H}, \mathrm{J}=6.3, \mathrm{~J}=11.7), 3.72(\mathrm{dd}, 1 \mathrm{H}, \mathrm{J}=$ $6.8, \mathrm{~J}=11.7), 4.00(\mathrm{~d}, 1 \mathrm{H}, \mathrm{J}=6.8), 4.04(\mathrm{dd}, 1 \mathrm{H}, \mathrm{J}=3.4, \mathrm{~J}=10.3), 4.62(\mathrm{brs}, 4 \mathrm{H}), 4.86(\mathrm{~d}, 1 \mathrm{H}, \mathrm{J}=3.4)$, $4.98(\mathrm{dd}, 1 \mathrm{H}, \mathrm{J}=3.4, \mathrm{~J}=10.3), 5.27(\mathrm{c}, 2 \mathrm{H}), 5.29(\mathrm{~d}, 1 \mathrm{H}, \mathrm{J}=3.4), 5.35(\mathrm{c}, 2 \mathrm{H}) 5.92(\mathrm{c}, 2 \mathrm{H})$

${ }^{13}$ C NMR (101 MHz, $\left.\mathrm{CDCl}_{3}\right): \delta 16.5,37.7,39.3,117.4,135.3,182.9$

IR (NaCl): $3084(\mathrm{w}), 1708$ (s), $1464(\mathrm{w}), 1419(\mathrm{w}), 1215(\mathrm{w}), 917(\mathrm{~m}) \mathrm{cm}^{-1}$.

\section{(R)-2-benzylpent-4-enoic acid}

${ }^{1}$ H NMR (500 MHz, CDCl3): $\delta 2.31(\mathrm{c}, 1 \mathrm{H}), 2.39$ (c, 1H), $2.80(\mathrm{c}, 2 \mathrm{H}), 2.98(\mathrm{c}, 1 \mathrm{H}), 5.10$ (c, 2H), 5.80 (c, 1H), $7.23(\mathrm{c}, 3 \mathrm{H}), 7.30(\mathrm{c}, 2 \mathrm{H})$

${ }^{13}$ C NMR (101 MHz, $\left.\mathrm{CDCl}_{3}\right): \delta 35.8,37.5,47.2,117.7,126.7,128.7,129.2,135.0,139.0,181.2$

IR (NaCl): 3064 (w), 3029 (w), 2924 (m), 1706 (s), 1642 (w), 1496 (w), 1285 (w), 918 (m) cm .

HRMS-ESI $(\mathrm{m} / \mathrm{z})$ : $\left[\mathrm{M}+\mathrm{NH}_{4}\right]^{+}$calcd for $\mathrm{C}_{12} \mathrm{H}_{18} \mathrm{NO}_{2} 208.1388$; found, 208.1337 


\section{(S)-2-((benzo[d]oxazol-2-yl)methyl)pent-4-enoic acid}

H NMR (500 MHz, $\left.\mathrm{CDCl}_{3}\right): \delta 2.45(\mathrm{ddd}, 1 \mathrm{H}, J=6.5, J=7.5, J=14.0), 2.55(\mathrm{~d} \mathrm{dd}, 1 \mathrm{H}, J=6.5, J=$ $7.5, J=14.0), 3.13(\mathrm{dd}, 1 \mathrm{H}, J=5.5, J=15.5), 3.24(\mathrm{ddd}, 1 \mathrm{H}, J=5.5, J=6.5, J=7.5), 3.33(\mathrm{dd}, 1 \mathrm{H}, J=$ $7.5, J=15.5), 5.09$ (d, 1H, $J=11), 5.12$ (d, 1H, $J=19), 5.79$ (ddd, 1H, $J=7.5, J=11, J=19), 7.29$ (c, 2H), 7.46 (c, 1H), 7.67 (c, 1H)

${ }^{13}$ C NMR $\left(101 \mathrm{MHz}_{\mathrm{CDCl}}\right): \delta 29.4,35.8,42.4,110.5,118.4,119.5,124.5,125.0,134.0,140.4,150.6$, $165.3,178.4$

IR (NaCl): 2976 (m), 2932 (m), 1736 (s), 1470 (w), 1144 (m), 1123 (m), 996 (m) cm . $^{-1}$

HRMS-ESI $(\mathrm{m} / \mathrm{z}):[\mathrm{M}+\mathrm{H}]^{+}$calcd for $\mathrm{C}_{13} \mathrm{H}_{14} \mathrm{NO}_{3} 232.0973$; found, 232.0983

\section{(S)-2-((butylcarbamoyl)methyl)pent-4-enoic acid}

${ }^{1}$ H NMR $\left(500 \mathrm{MHz}, \mathrm{CDCl}_{3}\right): \delta 3.43(\mathrm{~s}, 3 \mathrm{H}), 3.59(\mathrm{dd}, 1 \mathrm{H}, J=6.3, J=11.7), 3.72(\mathrm{dd}, 1 \mathrm{H}, J=6.8, J=$ 11.7), 4.00 (d, $1 \mathrm{H}, J=6.8), 4.04$ (dd, $1 \mathrm{H}, J=3.4, J=10.3$ ), 4.62 (brs, $4 \mathrm{H}), 4.86$ (d, 1H, $J=3.4), 4.98$ $(\mathrm{dd}, 1 \mathrm{H}, J=3.4, J=10.3), 5.27$ (c, 2H), 5.29 (d, 1H, $J=3.4), 5.35(\mathrm{c}, 2 \mathrm{H}) 5.92(\mathrm{c}, 2 \mathrm{H})$

${ }^{13}$ C NMR (101 MHz, $\left.\mathrm{CDCl}_{3}\right)$ : $\delta$ 55.9, 61.0, 69.1, 69.4, 69.9 71.3, 72.1, 77.2, 99.5, 119.1, 119.6, 131.2, $131.5,155.0,155.3$

IR (NaCl): $3077(\mathrm{w}), 1703(\mathrm{~s}), 1402(\mathrm{~m}), 1191(\mathrm{w}) \mathrm{cm}^{-1}$.

HRMS-ESI $(m / z):[\mathrm{M}+\mathrm{H}]^{+}$calcd for $\mathrm{C}_{11} \mathrm{H}_{19} \mathrm{NO}_{3} 201.1443$; found, 201.1441 


\section{Parallel Library Synthesis and Analysis}

\section{Selective loading of carbohydrate diol on macrobeads.}

The silylated macrobeads (574.4 mg, $1.43 \mathrm{mmol} / \mathrm{g}$, ca. $220 \mathrm{nmol}$ of Si/bead) were added to a $50 \mathrm{~mL}$ round bottom flask, which was capped with septum and flushed with dry nitrogen. Beads were swollen for $15 \mathrm{~min}$ in $\mathrm{CH}_{2} \mathrm{Cl}_{2}(5.0 \mathrm{~mL})$ and treated with trimethylsilyl chloride $(0.203 \mathrm{~mL}, 1.6 \mathrm{mmol}$, 2 equiv $)$ for 15 min. Subsequently beads were washed with $\mathrm{CH}_{2} \mathrm{Cl}_{2}(3 \mathrm{x})$ and suspended in a $4 \%$ solution (v/v) of trifluoromethanesufonic acid (10.6 mL, $4.79 \mathrm{mmol}, 6$ equiv) in $\mathrm{CH}_{2} \mathrm{Cl}_{2}$ for 15 min during which time the flask was shaken. The beads were filtered and washed with $\mathrm{CH}_{2} \mathrm{Cl}_{2}(5 \mathrm{x})$ and left suspended in $\mathrm{CH}_{2} \mathrm{Cl}_{2}(2$ $\mathrm{ml})$ and freshly distilled 2,6-lutidine ( $744 \mu \mathrm{L}, 6.39 \mathrm{mmol}, 8$ equiv) was added followed by a solution of the corresponding diol (Mw 362.3, $1.72 \mathrm{mmol}, 632.2 \mathrm{mg}$ ) in $\mathrm{CH}_{2} \mathrm{Cl}_{2} / \mathrm{DMF}(\mathrm{v} / \mathrm{v}, 1: 3,5 \mathrm{~mL}$ ) through a canular. The flask was then shaken for another $12 \mathrm{~h}$ with Thermolyne Roto Mix (type 50800, level 5). The beads were then filtered, suspended and rinsed with the sequence of solvents follows three times; $\mathrm{CH}_{2} \mathrm{Cl}_{2}$ (15 min), THF (15 min), THF/pros (1/1, $\left.15 \mathrm{~min}\right), \mathrm{CH}_{3} \mathrm{CN}$ (15 min) and then dried under mild reduced pressure. 10 parts of total amounts of beads were collected and dried under High vacuum (50 $\mathrm{mmHg}$ ) overnight and then lyophilized for $24 \mathrm{~h}$. The weight of the dried beads was $65.4 \mathrm{mg}$ (used for counting).

\section{Determination of loading level of compounds by gravimetric methods}

To macrobeads (50 mg, ca. 238 beads) pre-swollen with THF $(1.5 \mathrm{~mL})$ in a polypropylene eppendorf tube at $\mathrm{rt}$ under argon flow was added a freshly prepared solution of $5 \% \mathrm{HF} / \mathrm{pyr}$ in THF ( $1 \mathrm{~mL})$ The resulting mixture was then agitated VMR Scientific Vortex genie 2 for $3 \mathrm{~h}$ at $\mathrm{rt}$. The reaction was quenched with the addition of trimethoxysilane $(5.0 \mathrm{~mL})$. The resulting mixture was agitated for another 2 $\mathrm{h}$ and extracted with $\mathrm{CH}_{3} \mathrm{CN}(10 \mathrm{~mL} \times 5)$. The combined solutions were concentrated and purified by flash chromatography (10 g of silica gel, Hex/EtOAC: $2 / 1)$ to yield $7.8 \mathrm{mg}$ of diol ( $90 \mathrm{nmol} / \mathrm{bead})$.

This reaction was repeated with different amount of macrobeads and the followings are the results: $25 \mathrm{mg}$ (105 nmol/ bead), $50 \mathrm{mg}$ (92 nmol/bead), $75 \mathrm{mg}$ ( $88 \mathrm{nmol} /$ bead $), 100 \mathrm{mg}(94 \mathrm{nmol} / \mathrm{bead})$. 


\section{Carbamate formation of cyclic secondary alcohol on macrobeads.}

To a $2 \mathrm{~mL}$ Wheaton vial fitted with Teflon cap containing polymer-supported substrate $(24 \mathrm{mg}$, ca. 136 beads, $12.4 \mu \mathrm{mol}$ ) with DMAP (15 mg, $0.12 \mathrm{mmol}, 10$ equiv) in $20 \%$ pyridine in $\mathrm{CH}_{2} \mathrm{Cl}_{2}$ (v/v, 1.0

L) were added phenylisocyanate $(13.5 \mu \mathrm{L}, 0.12 \mathrm{mmol}, 10$ equiv). The reaction mixture was shaken with Thermolyne Roto Mix (type 50800, level 5) for $15 \mathrm{~h}$ at room temperature. The beads were then filtered, suspended and rinsed with the sequence of solvents follows three times; $\mathrm{CH}_{2} \mathrm{Cl}_{2}$ (15 min), THF (15 min), $\mathrm{THF} / \mathrm{PrOH}(1 / 1,15 \mathrm{~min}), \mathrm{DMF}(15 \mathrm{~min}), \mathrm{CH}_{3} \mathrm{CN}$ (15 min) and then dried under mild reduced pressure to yield $31 \mathrm{mg}$ of beads (uncorrected).

\section{Determination of selectivity of loading step and quantity of materials on macrobeads}

Half of the resulting macrobeads $(15.5 \mathrm{mg}$, ca. 68 beads) pre-swollen with THF $(0.5 \mathrm{~mL})$ in a polypropylene eppendorf tube at $\mathrm{rt}$ under argon flow was added a freshly prepared solution of $5 \% \mathrm{HF} / \mathrm{pyr}$ in THF $(0.3 \mathrm{~mL})$ The resulting mixture was then agitated VMR Scientific Vortex genie 2 for $3 \mathrm{~h}$ at $\mathrm{rt}$. The reaction was quenched with the addition of triethoxysilane $(1.5 \mathrm{~mL})$. The resulting mixture was agitated for another $2 \mathrm{~h}$ and extracted with $\mathrm{CH}_{3} \mathrm{CN}(1.0 \mathrm{~mL}$ x 5). The combined solution was concentrated in vacuo and diluted with $\mathrm{CH}_{3} \mathrm{CN}(1.0 \mathrm{~mL})$. The solution was concentrated again and then dissolved to make $2.0 \mathrm{~mL}$ solution of $\mathrm{CH}_{3} \mathrm{CN}$.

$10.0 \mathrm{mg}$ of authentic sample prepared in solution phase with known purity ( $>95 \% 1 \mathrm{H} \mathrm{NMR,}>99 \%$ by HPLC) was dissolved in $3.0 \mathrm{~mL}$ of $\mathrm{CH}_{3} \mathrm{CN}$. This standard solution was diluted two, three fold and four fold to generate standard curve twice. Interpolation of measured concentrations were done twice in stock solution concentrations and two fold diluted samples, which provided equivalent $(85 \pm 7 \mathrm{nmol} / \mathrm{bead}$, $25>1$ ) results. 


\section{Deprotection of bis-allyloxycarbonate groups on macrobeads.}

To a $2 \mathrm{~mL}$ Wheaton vial fitted with Teflon cap containing substrate-bound macrobeads $(50 \mathrm{mg}, 25$ $\mu \mathrm{mol})$ pre-swollen in THF $(0.3 \mathrm{~mL})$ for $30 \mathrm{~min}$ was added thiosalicylic acid $(19.3 \mathrm{mg}, 0.125 \mathrm{mmol}, 5$ equiv) and $\mathrm{Pd}\left(\mathrm{PPh}_{3}\right)_{4}(26.0 \mathrm{mg}, 90 \mathrm{~mol} \%)$ under positive argon pressure. The reaction mixture was shaken with Thermolyne Roto Mix (type 50800, level 5) at room temperature for $13 \mathrm{~h}$. The beads were then filtered, suspended and rinsed with the sequence of solvents follows three times; THF (15 min), $\mathrm{THF} / \mathrm{PPrOH}$ (1/1, $15 \mathrm{~min}), \mathrm{THF} / \mathrm{H}_{2} \mathrm{O}$ (1/1, $\left.15 \mathrm{~min}\right)$, DMF (15 min), $\mathrm{CH}_{3} \mathrm{CN}$ (15 min) and then dried under mild reduced pressure to give $45 \mathrm{mg}$ of beads (uncorrected).

\section{bis-pentenoates formation on macrobeads.}

To a $2 \mathrm{~mL}$ Wheaton vial fitted with Teflon cap containing substrate-bound macrobeads (12 mg, 6 $\mu \mathrm{mol})$ in dichloromethane $(0.5 \mathrm{~mL})$ was added 2-methylpentenoic acid (7.1 $\mathrm{mg}, 0.06 \mathrm{mmol}, 10$ equiv). After $10 \mathrm{~min}$, diisopropylcarboimide $(3.9 \mu \mathrm{L}, 24 \mu \mathrm{mol}, 4$ equiv) and DMAP $(0.8 \mathrm{mg})$ was added to the reaction mixture subsequently. The reaction mixture was shaken with Thermolyne Roto Mix (type 50800, level 5) for $15 \mathrm{~h}$ at room temperature. The beads were transferred to $2 \mathrm{~mL}$ fritted polypropylene Bio-Spin chromatography columns then filtered, suspended and rinsed with the sequence of solvents follows; THF (15 min), THF/iPrOH (1/1, $15 \mathrm{~min}), \mathrm{THF} / \mathrm{H}_{2} \mathrm{O}$ (1/1, $\left.15 \mathrm{~min}\right), \mathrm{DMF}$ (15 min), $\mathrm{CH}_{2} \mathrm{Cl}_{2}$ (15 min) and then dried through the flow of nitrogen on a Vac-Man laboratory Vacuum Manifold (Promega A7231) for $2 \mathrm{~h}$.

The fritted column was capped and the substrate-bound macrobeads exposed to same conditions again. The beads were then filtered, suspended and rinsed with the sequence of solvents follows three times; THF (15 min), THF/iPrOH (1/1, $15 \mathrm{~min}), \mathrm{THF} / \mathrm{H}_{2} \mathrm{O}$ (1/1, $\left.15 \mathrm{~min}\right), \mathrm{DMF}$ (15 min), $\mathrm{CH}_{3} \mathrm{CN}$ (15 $\mathrm{min}$ ) and then dried under mild reduced pressure to yield $14 \mathrm{mg}$ of beads (uncorrected).

\section{Ring closing metathesis reaction on macrobeads.}

To a $2 \mathrm{~mL}$ Wheaton vial fitted with Teflon cap containing polymer-supported substrate ( $2 \mathrm{mg}$, ca. $0.9 \mu \mathrm{mol})$ pre-swollen in $\mathrm{CH}_{2} \mathrm{Cl}_{2}(0.3 \mathrm{~mL})$ for 30 min was added freshly made Grubbs' catalyst solution $\left(0.9 \mathrm{~mL}, 1 \mathrm{mM}\right.$ in $\mathrm{CH}_{2} \mathrm{Cl}_{2}$ ). The reaction mixture was sluggishly sealed and shaken every $30 \mathrm{~min}$ manually at $40{ }^{\circ} \mathrm{C}$ for $4 \mathrm{~h}$. The beads were then filtered, suspended and rinsed with the sequence of solvents follows two times; $\mathrm{CH}_{2} \mathrm{Cl}_{2}$ (15 min), THF (15 min), THF/iPrOH (1/1, $15 \mathrm{~min}$ ), DMSO (15 min), $\mathrm{CH}_{3} \mathrm{CN}$ (15 min) and dried under mild reduced pressure. Depending on the color of beads, published washing protocols are pursued. ${ }^{14}$ 


\section{Quality check of Parallel Split-split Library members}

\begin{tabular}{|c|c|c|c|c|}
\hline Compound & $\begin{array}{l}\text { \% Purity } \\
\text { UV }(254 \mathrm{~nm})\end{array}$ & Ion & Calculated & Observed \\
\hline I_3_4_B & 95 & $\mathrm{M}+\mathrm{NH}_{4}^{+}$ & 692 & 692 \\
\hline I_3_4_M & $>90$ & $\mathrm{M}+\mathrm{NH}_{4}^{+}$ & 720 & 720 \\
\hline II_1_3_B & $>92$ & $\mathrm{M}+\mathrm{NH}_{4}^{+}$ & 496 & 496 \\
\hline II_1_3_M & $>90$ & $\mathrm{M}+\mathrm{NH}_{4}^{+}$ & 524 & 524 \\
\hline III_3_3_B & 93 & $\mathrm{M}+\mathrm{NH}_{4}^{+}$ & 504 & 504 \\
\hline IV_1_2_B & $>85$ & $\mathrm{M}+\mathrm{NH}_{4}^{+}$ & 496 & 496 \\
\hline IV_1_2_M & $>80$ & $\mathrm{M}+\mathrm{NH}_{4}^{+}$ & 524 & 524 \\
\hline I_1_3_B & $>90$ & $\mathrm{M}+\mathrm{NH}_{4}^{+}$ & 496 & 496 \\
\hline I_1_3_M & $>86$ & $\mathrm{M}+\mathrm{NH}_{4}^{+}$ & 524 & 524 \\
\hline II_1_2_B & $>87$ & $\mathrm{M}+\mathrm{NH}_{4}^{+}$ & 496 & 496 \\
\hline II_1_2_M & $>90$ & $\mathrm{M}+\mathrm{NH}_{4}^{+}$ & 524 & 524 \\
\hline IV_2_4_B & $>82$ & $\mathrm{M}+\mathrm{NH}_{4}^{+}$ & 531 & 531 \\
\hline III_1_2_B & $>85$ & $\mathrm{M}+\mathrm{NH}_{4}^{+}$ & 496 & 496 \\
\hline III_1_2_M & $>85$ & $\mathrm{M}+\mathrm{NH}_{4}^{+}$ & 524 & 524 \\
\hline V_2_1_B & 92 & $\mathrm{M}+\mathrm{NH}_{4}^{+}$ & 481 & 481 \\
\hline II_2_8_B & $>81$ & $\mathrm{M}+\mathrm{NH}_{4}^{+}$ & 679 & 679 \\
\hline II_1_6_B & 90 & $\mathrm{M}+\mathrm{NH}_{4}^{+}$ & 715 & 715 \\
\hline IV_1_3_B & $>70$ & $\mathrm{M}+\mathrm{NH}_{4}^{+}$ & 496 & 496 \\
\hline IV_1_3_M & $>79$ & $\mathrm{M}+\mathrm{NH}_{4}^{+}$ & 524 & 524 \\
\hline III_1_3_B & $>85$ & $\mathrm{M}+\mathrm{NH}_{4}^{+}$ & 496 & 496 \\
\hline III_1_3_M & $>80$ & $\mathrm{M}+\mathrm{NH}_{4}^{+}$ & 524 & 524 \\
\hline III_3_6_B & 87 & $\mathrm{M}+\mathrm{NH}_{4}^{+}$ & 774 & 774 \\
\hline II_3_6_B & $>83$ & $\mathrm{M}+\mathrm{NH}_{4}^{+}$ & 774 & 774 \\
\hline I_3_6_B & 90 & $\mathrm{M}+\mathrm{NH}_{4}^{+}$ & 774 & 774 \\
\hline III_3_7_B & $>82$ & $\mathrm{M}+\mathrm{NH}_{4}^{+}$ & 774 & 774 \\
\hline III_2_6_B & 90 & $\mathrm{M}+\mathrm{NH}_{4}^{+}$ & 715 & 715 \\
\hline III_4_9_B & $>70$ & $\mathrm{M}+$ & 701 & 701 \\
\hline IV_1_1_B & $>93$ & $\mathrm{M}+\mathrm{NH}_{4}^{+}$ & 496 & 496 \\
\hline IV_1_1_M & $>95$ & $\mathrm{M}+\mathrm{NH}_{4}^{+}$ & 524 & 524 \\
\hline V_1_3_B & $>95$ & $\mathrm{M}+\mathrm{NH}_{4}^{+}$ & 496 & 496 \\
\hline V_1_3_M & $>92$ & $\mathrm{M}+\mathrm{NH}_{4}^{+}$ & 524 & 524 \\
\hline VI_1_2_B & $>72$ & $\mathrm{M}+\mathrm{NH}_{4}^{+}$ & 496 & 496 \\
\hline VI_1_2_M & $>79$ & $\mathrm{M}+\mathrm{NH}_{4}^{+}$ & 524 & 524 \\
\hline
\end{tabular}




\begin{tabular}{|c|c|c|c|c|}
\hline V_1_2_B & $>85$ & $\mathrm{M}+\mathrm{NH}_{4}^{+}$ & 496 & 496 \\
\hline V_1_2_M & $>88$ & $\mathrm{M}+\mathrm{NH}_{4}^{+}$ & 524 & 524 \\
\hline III_4_8_B & $>92$ & $\mathrm{M}+\mathrm{NH}_{4}^{+}$ & 719 & 719 \\
\hline III_4_8_M & $>95$ & $\mathrm{M}+\mathrm{NH}_{4}^{+}$ & 747 & 747 \\
\hline I_2_6_B & $>88$ & $\mathrm{M}+\mathrm{NH}_{4}^{+}$ & 715 & 715 \\
\hline I_1_6_B & $>90$ & $\mathrm{M}+\mathrm{NH}_{4}^{+}$ & 730 & 730 \\
\hline VI_1_3_B & $>84$ & $\mathrm{M}+\mathrm{NH}_{4}^{+}$ & 496 & 496 \\
\hline VI_1_3_M & $>78$ & $\mathrm{M}+\mathrm{NH}_{4}^{+}$ & 524 & 524 \\
\hline I_2_1_M & 95 & $\mathrm{M}+\mathrm{NH}_{4}^{+}$ & 481 & 48 \\
\hline I_2_1_B & 95 & $\mathrm{M}+\mathrm{NH}_{4}^{+}$ & 453 & 45 \\
\hline
\end{tabular}

\# Details about library members and their calculated chemical descriptors are found in the following excel file: SuppInfo_III.xls 


\section{Representative compounds for full characterization}

The followings are compounds generated in parallel to collect the full spectroscopic characterization of a number of representative compounds from library members. The reactions were done in larger scale (20 mg to $100 \mathrm{mg}$ of macrobeads) and subsequent purification was followed if necessary. Both crude and purified spectra are shown in some cases (part II) for comparison.

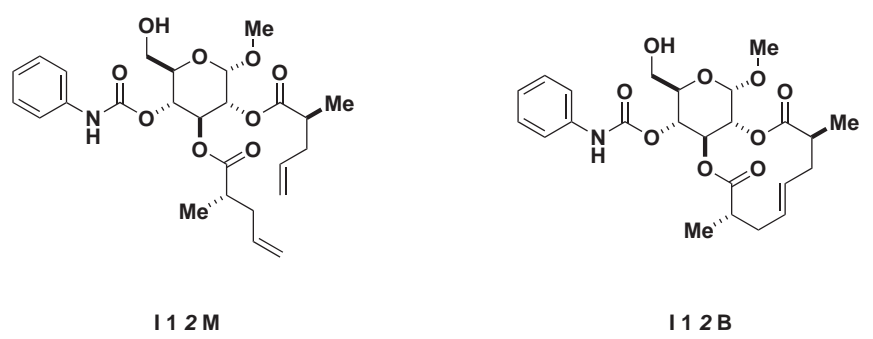

\section{[I_1_2_M]}

$\mathbf{t}_{\mathbf{R}}$ (LC/MS): $9.0 \mathrm{~min}$.

${ }^{1}$ H NMR (500 MHz, $\left.\mathrm{CDCl}_{3}\right): \delta 1.06(\mathrm{~d}, 3 \mathrm{H}, J=6.5), 1.15$ (d, 3H, $\left.J=6.5\right), 2.06-2.19$ (c, 2H), 2.33-2.41 (c, 2H), 2.45-2.61 (c, 2H), $2.76(\mathrm{br} \mathrm{s}, 1 \mathrm{H}), 3.41(\mathrm{~s}, 3 \mathrm{H}), 3.77$ (c, 3H), 4.89 (dd, 1H, J=4.0, J=10.0), 4.91 (app t,$J=10.0), 5.01$ (d, 1H, $J=4.0), 4.97-5.08$ (c, 4H), 5.65 (app t, 1H, $J=10.0), 5.60-5.74(\mathrm{c}, 2 \mathrm{H})$, 6.76 (brs, 1H), 7.10 (t, 1H, $J=7.5), 7.33$ (c, $4 \mathrm{H})$

${ }^{13}$ C NMR (101 MHz, $\left.\mathrm{CDCl}_{3}\right): \delta 16.7,17.0,37.4,37.6,39.4,39.5,55.8,61.1,68.8,69.5,70.2,71.2,97.0$, 117.3, 117.4, 119.0, 124.4, 129.4 (2), 135.2, 135.3, 135.6, 137.2, 153.1, 175.3, 175.6

IR (NaCl): 3342(w), 2975(w), 2937(w), 1745(s), 1602(w), 1541(w), $1445(w), 1125(\mathrm{~m}), 917(\mathrm{w}) \mathrm{cm}^{-1}$. HRMS-ESI $(\mathrm{m} / \mathrm{z}):[\mathrm{M}+\mathrm{H}]^{+}$calcd for $\mathrm{C}_{26} \mathrm{H}_{36} \mathrm{NO}$, 506.2390; found, 506.2381

\section{[I_1_2_B]}

$\mathbf{t}_{\mathbf{R}}(\mathrm{LC} / \mathrm{MS}): 7.8 \mathrm{~min}$

${ }^{1} \mathbf{H} \operatorname{NMR}\left(500 \mathrm{MHz}, \mathrm{CDCl}_{3}\right): \delta 1.01(\mathrm{~d}, 3 \mathrm{H}, J=6.5), 1.17(\mathrm{~d}, 3 \mathrm{H}, J=7.0), 2.05-2.18(\mathrm{c}, 4 \mathrm{H}), 2.21$ (ddd, $1 \mathrm{H}, J=2.5, J=7.0, J=15.5$ ), 2.57 (ddd, $1 \mathrm{H}, J=2.5, J=7.0, J=15.5$ ), 2. (brs, $1 \mathrm{H}), 3.43$ (s, 3H), 3.62 (app t, $1 \mathrm{H}, J=6.5), 4.86(\mathrm{~d}, 1 \mathrm{H}, J=4.0), 4.95$ (app t,$J=9.5), 5.09(\mathrm{dd}, 1 \mathrm{H}, J=4.0, J=10.5), 5.32-5.42$ (c, 2H), 5.61 (app t, $1 \mathrm{H}, J=10.5$ ), 6.73 (brs, 1H), 7.10 (t, 1H, $J=7.0$ ), 7.28-7.40 (c, 4H)

${ }^{13}$ C NMR (101 MHz, $\left.\mathrm{CDCl}_{3}\right): \delta 18.0,18.2,38.6,39.1,40.3,40.7,55.7,61.2,69.2,69.8,69.9,70.3,97.5$, 119.0, 124.4, 127.6, 129.3, $129.4(2), 129.9,137.2,153.1,175.9,176.4$

IR (NaCl): 3340(w), 2933(w), 1745(s), 1602(w), 1541(w), $1444(\mathrm{w}), 1222(\mathrm{~m}), 1053(\mathrm{~m}), 756(\mathrm{w}) \mathrm{cm}^{-1}$. HRMS-ESI $(\mathrm{m} / \mathrm{z}):[\mathrm{M}+\mathrm{H}]^{+}$calcd for $\mathrm{C}_{24} \mathrm{H}_{32} \mathrm{NO} 9,478.2077$; found, 478.2072.

Yields: 20 (/32) mg of [I_1_2_M]-linked beads provided 5.6 (9.9) mg of pure [I_1_2_B] -> 85 \%

* diene-supported beads: 5300 beads/g (used for the rest of calculations) 


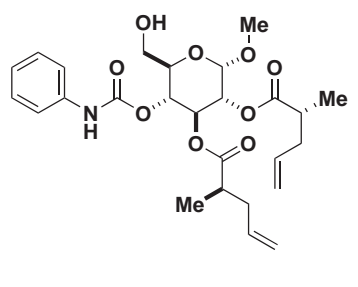

I1 3 B

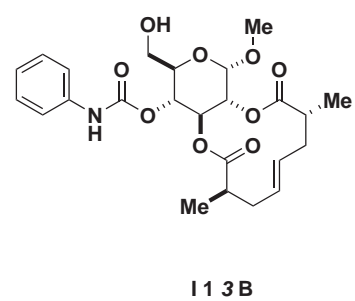

[I_1_3_M]

$\mathbf{t}_{\mathbf{R}}$ (LC/MS): $9.0 \mathrm{~min}$

${ }^{1}$ H NMR $\left(500 \mathrm{MHz}, \mathrm{CDCl}_{3}\right): \delta 1.06$ (d, 3H, $\left.J=6.7\right), 1.20$ (d, 3H, $\left.J=6.8\right), 2.10$ (ddd, $1 \mathrm{H}, J=6.8, J=7.0$, $J=13.5$ ), 2.21 (ddd, $1 \mathrm{H}, J=2.5, J=6.8, J=13.5$ ), 2.38 (c, $2 \mathrm{H}), 2.78$ (c, $2 \mathrm{H}), 3.42(\mathrm{~s}, 3 \mathrm{H}), 3.62$ (dd, $1 \mathrm{H}$, $J=7.5, J=11.5$ ), 3.73 (dd, $1 \mathrm{H}, J=6.5, J=11.5), 4.10$ (app t,$J=6.8), 4.87$ (d, $1 \mathrm{H}, J=3.5), 5.30$ (dd, $J$ $=3.5, J=11.0), 5.37(\mathrm{~d}, 1 \mathrm{H}, J=3.5), 5.43-5.50(\mathrm{c}, 2 \mathrm{H}), 5.50(\mathrm{dd}, 1 \mathrm{H}, J=3.5, J=11.0), 6.90$ (brs, $1 \mathrm{H})$, $7.12(\mathrm{t}, 1 \mathrm{H}, J=8.0), 7.35(\mathrm{t}, 2 \mathrm{H}, J=8.0), 7.41$ (d, $1 \mathrm{H}, J=8.0)$

${ }^{13} \mathbf{C}$ NMR $\left(101 \mathrm{MHz}, \mathrm{CDCl}_{3}\right): \delta 16.5\left(\mathrm{CH}_{3}\right), 16.8\left(\mathbf{C H}_{3}\right), 37.5\left(\mathbf{C H}_{2}\right), 37.7\left(\mathbf{C H}_{2}\right), 38.9(\mathbf{C H}), 39.4(\mathbf{C H})$, 55.8(CH), 61.1 $\left(\mathrm{CH}_{2}\right), 69.6(\mathbf{C H}), 69.8(\mathrm{CH}), 70.0(\mathbf{C H}), 71.2(\mathrm{CH}), 97.1(\mathrm{CH}), 117.2\left(\mathrm{CH}_{2}\right), 117.3\left(\mathrm{CH}_{2}\right)$, 119.0(CH), 124.3(CH), $129.4\left(2 \_\mathbf{C H}\right), 135.1(\mathbf{C H}), 135.2(\mathbf{C H}), 137.3(\mathbf{C}), 153.1(\mathbf{C O}), 175.4(\mathbf{C O})$, $175.6(\mathrm{CO})$

IR (NaCl): 3342(w), 3077 (w), 2976(w), 1746(s), 1603(w), 1541(m), 1445 (w), 1216(m), 758(w) $\mathrm{cm}^{-1}$. HRMS-ESI $(\mathrm{m} / \mathrm{z}):[\mathrm{M}+\mathrm{H}]^{+}$calcd for $\mathrm{C}_{26} \mathrm{H}_{36} \mathrm{NO}$, 506.2390; found, 506.2381

\section{[I_1_3_B]}

$\mathbf{t}_{\mathbf{R}}$ (LC/MS): $7.8 \mathrm{~min}$

${ }^{1} \mathbf{H}$ NMR ( $500 \mathrm{MHz}, \mathrm{CDCl}_{3}$ ): $\delta 1.10$ (d, $\left.3 \mathrm{H}, J=7.0\right), 1.17$ (d, 3H, $\left.J=7.0\right), 2.11$ (ddd, $1 \mathrm{H}, J=4.5, J=5.5$, $J=13.5$ ), 2.18 (ddd, $1 \mathrm{H}, J=6.0, J=9.0, J=15$ ), 2.28 (ddd, $1 \mathrm{H}, J=4.5, J=5.5, J=15$ ), 2.45 (ddd, $1 \mathrm{H}, J$ $=4.5, J=8.5, J=13.5$ ), 2.60 (brs, $1 \mathrm{H}), 3.41$ (s, 3H), 3.70-3.82 (c, 3H), 4.85 (dd, $1 \mathrm{H}, J=3.5, J=10.0$ ), 4.94 (d ,1H, $J=3.5$ ), 5.00 (app t, $1 \mathrm{H}, J=10.0$ ), 5.41 (ddd, $1 \mathrm{H}, J=5.5, J=8.5, J=15.5$ ), 5.48 (ddd, $1 \mathrm{H}$, $J=6.0, J=9.0, J=15.5$ ), 5.63 (app t, $1 \mathrm{H}, J=10.0$ ), 6.72 (brs, $1 \mathrm{H}$ ), 7.10 (t, $1 \mathrm{H}, J=7.0$ ), 7.28-7.40 (c, $4 \mathrm{H})$

${ }^{13}$ C NMR $\left(101 \mathrm{MHz}, \mathrm{CDCl}_{3}\right): \delta 14.7\left(\mathrm{CH}_{3}\right), 16.6\left(\mathrm{CH}_{3}\right), 36.5\left(\mathrm{CH}_{2}\right), 36.9\left(\mathrm{CH}_{2}\right), 40.6(\mathbf{C H}), 40.7(\mathbf{C H})$, 55.8(CH), 61.3( $\left(\mathrm{CH}_{2}\right), 69.1(\mathrm{CH}), 69.8(\mathrm{CH}), 69.9(\mathrm{CH}), 71.0(\mathrm{CH}), 97.4(\mathrm{CH}), 119.0(\mathrm{CH}), 124.3(\mathrm{CH})$, 127.2(CH), 129.4(2_CH) 129.5(2_CH), 137.3(C), 153.0(CO), 174.4(CO), 174.7(CO)

IR (NaCl): 3336(w), 2936(w), 1738(s), 1602(w), 1541(w), 1444 (w), 1248 (w), 1053(s), 756(w) $\mathrm{cm}^{-1}$.

HRMS-ESI $(\mathrm{m} / \mathrm{z}):[\mathrm{M}+\mathrm{H}]^{+}$calcd for $\mathrm{C}_{2} 4 \mathrm{H}_{32} \mathrm{NO}$, 478.2077; found, 478.2072 .

Yields: $25 \mathrm{mg}$ of [I_1_3_M]-linked beads provided $7.4 \mathrm{mg}$ of pure [I_1_3_B]: $90 \%$ 


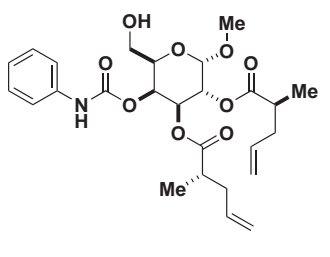

II 12 M

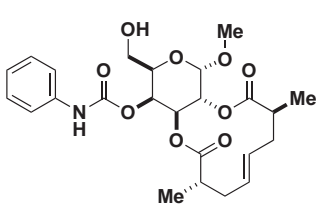

II 12 B

\section{[II_1_2_M]}

$\mathbf{t}_{\mathrm{R}}(\mathrm{LC} / \mathrm{MS}): 12.9 \mathrm{~min}$

${ }^{1}$ H NMR $\left.500 \mathrm{MHz}, \mathrm{CDCl}_{3}\right): \delta 1.08(\mathrm{~d}, 3 \mathrm{H}, J=7.0), 1.16(\mathrm{~d}, 3 \mathrm{H}, J=7.0), 2.05-2.22(\mathrm{c}, 2 \mathrm{H}), 2.30-2.60(\mathrm{c}$, $4 \mathrm{H}), 3.41(\mathrm{~s}, 3 \mathrm{H}), 3.61(\mathrm{dd}, 1 \mathrm{H}, J=7.0, J=12.0), 3.71(\mathrm{dd}, 1 \mathrm{H}, J=7.0, J=12.0), 4.12(\mathrm{~m}, 1 \mathrm{H}), 4.95-$ 5.08 (c, 4H), 4.99 (brd, 1H, $J=3.5$ ), 5.24 (dd, 1H, $J=3.5, J=10.0$ ), 5.44 (brs, 1H), 5.45 (c, 1H), 5.60$5.75(\mathrm{~m}, 2 \mathrm{H}), 6.94$ (brs, $1 \mathrm{H}), 7.11(\mathrm{t}, 1 \mathrm{H}, J=3.0), 7.32-7.41(\mathrm{c}, 4 \mathrm{H})$

${ }^{13}$ C NMR (101 MHz, $\left.\mathrm{CDCl}_{3}\right): \delta 16.6,16.8,37.5,37.7,39.4,39.5,55.8,60.8,67.9,68.6,69.1,70.1,97.4$, $117.3,117.3,118.9,124.3,128.2,129.3,129.4,135.3,135.3,137.4,153.5,175.0,175.8$

IR (NaCl): 3435 (w), 2977 (w), 1738 \&s), 1553 (w), 1228 (w), 1136 (w), 1043 (m), 763 (w) $\mathrm{cm}^{-1}$.

HRMS-ESI $(\mathrm{m} / \mathrm{z}):[\mathrm{M}+\mathrm{H}]^{+}$calcd for $\mathrm{C}_{26} \mathrm{H}_{36} \mathrm{NO}$, 506.2390; found, 506.2382

\section{[II_1_2_B]}

$\mathbf{t}_{\mathbf{R}}(\mathrm{LC} / \mathrm{MS}): 10.6 \mathrm{~min}$

${ }^{1}$ H NMR (500 MHz, $\mathrm{CDCl}_{3}$ ): $\delta 1.14$ (d, 3H, $\left.J=6.8\right), 1.18$ (d, 3H, $\left.J=6.8\right), 2.05-2.20(\mathrm{c}, 4 \mathrm{H}), 2.37$ (ddd, $1 \mathrm{H}, J=6.8, J=7.0, J=13.5$ ), 2.50 (brs, $1 \mathrm{H}), 2.59$ (ddd, $1 \mathrm{H}, J=2.5, J=6.8, J=13.5$ ), 3.44 (s, $3 \mathrm{H}), 3.62$ (dd, 1H, $J=7.0, J=11.5$ ), 3.73 (dd, 1H, $J=6.5, J=11.5$ ), 4.11 (app t,$J=7.0), 4.85$ (d, 1H, $J=3.5$ ), $5.30-5.38$ (c, 3H), 5.39 (dd, 1H, $J=4.0, J=11.5), 5.51$ (dd, 1H, $J=11.0), 6.90$ (brs, 1H), 7.11 (t, 1H, $J$ $=7.5), 7.34(\mathrm{t}, 2 \mathrm{H}, J=7.5), 7.42(\mathrm{~d}, 1 \mathrm{H}, J=7.5)$

${ }^{13}$ C NMR (101 MHz, $\left.\mathrm{CDCl}_{3}\right): \delta 17.7,18.1,38.9,39.2,40.3,40.4,55.7,60.9,66.9,67.4,69.1$ (2), 97.9, $118.8,124.2,129.4(3), 129.8(2), 137.5,153.5,175.6,176.4$

IR (NaCl): 3337 (w), 2925 (m), 2853 (w), 1740 (s), 1599 (w), 1445 (w), 1043 (m), 755 (w) cm .

HRMS-ESI $(\mathrm{m} / \mathrm{z}):[\mathrm{M}+\mathrm{H}]^{+}$calcd for $\mathrm{C}_{24} \mathrm{H}_{32} \mathrm{NO} 9,478.2077$; found, 478.2073.

Yields: $40 \mathrm{mg}$ of [II_1_2_M]-linked beads provided $11.5 \mathrm{mg}$ of pure [II_1_2_B]: 87 \% 


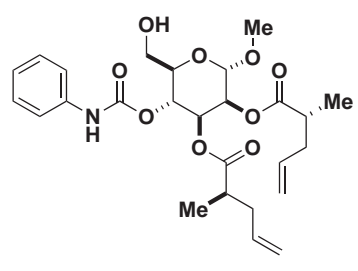

III 13 M

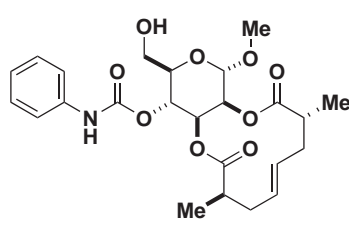

III 13 B

[II_1_3_M]

$\mathbf{t}_{\mathbf{R}}(\mathrm{LC} / \mathrm{MS}): 12.7 \mathrm{~min}$

${ }^{1} \mathbf{H}$ NMR(500 MHz, $\left.\mathrm{CDCl}_{3}\right): \delta 1.08(\mathrm{~d}, 3 \mathrm{H}, J=7.0), 1.14(\mathrm{~d}, 3 \mathrm{H}, J=6.5), 2.11$ (app quint, $\left.1 \mathrm{H}, J=7.0\right)$, 2.21 (app quint, $1 \mathrm{H}, J=7.0$ ), 2.33-2.49 (c, 2H), 2.58 (app dt,1H, $J=7.0, J=14.0), 3.41$ (s, 3H), 3.61 (m, $1 \mathrm{H}), 3.69(\mathrm{~m}, 1 \mathrm{H}), 4.10(\mathrm{t}, 1 \mathrm{H}, J=7.0), 4.95(\mathrm{~d}, 1 \mathrm{H}, J=3.5), 4.95-5.08(\mathrm{c}, 4 \mathrm{H}), 5.24$ (dd, $1 \mathrm{H}, J=3.5, J$ $=10.5), 5.44(\operatorname{app~t}, 1 \mathrm{H}, J=3.5), 5.50(\mathrm{dd}, 1 \mathrm{H}, J=3.5, J=10.5), 5.61-5.76(\mathrm{c}, 2 \mathrm{H}), 6.90(\mathrm{brs}, 1 \mathrm{H}), 7.11$ (t, 1H, $J=7.5), 7.34(\mathrm{t}, 2 \mathrm{H}, J=7.0), 7.40(\mathrm{~m}, 2 \mathrm{H})$

${ }^{13}$ C NMR (101 MHz, $\left.\mathrm{CDCl}_{3}\right): \delta$ 16.6, 16.7, 37.7, 37.8, 39.1, 39.3, 55.8, 60.7, 67.8, 68.5, 69.0, 70.3, 97.5, 117.2, 117.3, 118.9, 124.3, $129.4(3), 135.1,135.3,137.3,153.6,175.1,175.9$

IR (NaCl): 3081 (w), 2936 (w), 1734 (s), 1713 (s), 1446 (w), 1229 (m), 1134 (m), 764 (w) cm .

HRMS-ESI $(\mathrm{m} / \mathrm{z}):[\mathrm{M}+\mathrm{H}]^{+}$calcd for $\mathrm{C}_{26} \mathrm{H}_{36} \mathrm{NO}$, 506.2390; found, 506.2388.

\section{[II_1_3_B]}

$\mathbf{t}_{\mathbf{R}}(\mathrm{LC} / \mathrm{MS}): 11.2 \mathrm{~min}$

${ }^{1} \mathbf{H} \operatorname{NMR}\left(500 \mathrm{MHz}, \mathrm{CDCl}_{3}\right): \delta 1.06(\mathrm{~d}, 3 \mathrm{H}, J=7.0), 1.20(\mathrm{~d}, 3 \mathrm{H}, J=7.0), 2.10(\mathrm{ddd}, 1 \mathrm{H}, J=6.8, J=7.0$, $J=13.5), 2.21(\mathrm{ddd}, 1 \mathrm{H}, J=2.5, J=6.8, J=13.5), 2.38(\mathrm{c}, 2 \mathrm{H}), 2.78(\mathrm{c}, 2 \mathrm{H}), 3.42(\mathrm{~s}, 3 \mathrm{H}), 3.62(\mathrm{c}, 1 \mathrm{H})$, $3.73(\mathrm{c}, 1 \mathrm{H}), 4.11($ app t,$J=6), 4.87(\mathrm{~d}, 1 \mathrm{H}, J=3.5), 5.30(\mathrm{dd}, J=3.5, J=11.0), 5.36(\mathrm{~d}, 1 \mathrm{H}, J=3.5)$, $5.43-5.50(\mathrm{c}, 2 \mathrm{H}), 5.50(\mathrm{dd}, 1 \mathrm{H}, J=3.5, J=11.0), 6.94(\mathrm{brs}, 1 \mathrm{H}), 7.11$ (t, 1H, $J=7.5), 7.34$ (t, 2H, $J=$ 7.5), $7.41(\mathrm{~d}, 1 \mathrm{H}, J=7.5)$

${ }^{13}$ C NMR (101 MHz, $\left.\mathrm{CDCl}_{3}\right): \delta 15.3,15.4,36.7,36.8,40.6,40.7,55.9,60.9,67.2,67.9,69.1,70.4,97.9$, 118.9, 124.2, 128.1, 128.2, 129.4, 129.4 (2), 137.4, 153.5, 173.7, 174.8

IR (NaCl):3273 (w), 2931(m), 2852 (w), 1736 (s), 1602 (w), $1445(\mathrm{w}), 1219(\mathrm{~m}), 1139(\mathrm{~m}), 756(\mathrm{w}) \mathrm{cm}^{-1}$.

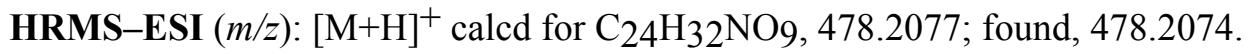

Yields: $34 \mathrm{mg}$ of [II_1_3_M]-linked beads provided $10.2 \mathrm{mg}$ of pure [II_1_3_B]: $87 \%$ 


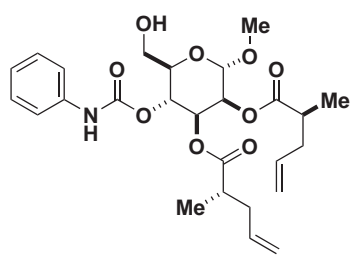

III 12 M

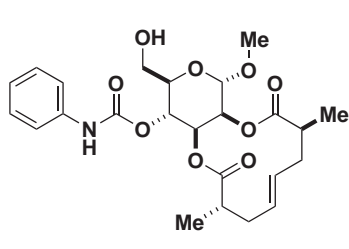

III 12 B

\section{[III_1_2_M]}

$\mathbf{t}_{\mathbf{R}}(\mathrm{LC} / \mathrm{MS}): 9.1 \mathrm{~min}$

${ }^{1}$ H NMR $\left(500 \mathrm{MHz}, \mathrm{CDCl}_{3}\right): \delta 1.05(\mathrm{~d}, 3 \mathrm{H}, J=7.0), 1.22(\mathrm{~d}, 3 \mathrm{H}, J=7.0), 2.10(\mathrm{ddd}, 1 \mathrm{H}, J=7.0, J=7.5$, $J=14.5), 2.22(\mathrm{ddd}, 1 \mathrm{H}, J=7.0, J=7.5, J=14.5), 2.35(\mathrm{ddd}, 1 \mathrm{H}, J=7.0, J=7.5, J=14.5), 2.46$ (dd, $1 \mathrm{H}, J=7.0, J=14.0$ ), 2.47 (c, 1H), 2.67 (dd, 1H, $J=7.0, J=14.0$ ), 3.42 (s, 3H), 3.76-3.82 (c, 3H), 4.72 (d, $1 \mathrm{H}, J=1.5), 4.94-5.01$ (c, 2H), 5.04-5.12 (c, 2H), 5.24 (app t, 1H, J = 10.0), 5.25 (dd, $J=1.5, J=3.5$ ), 5.51 (dd, $1 \mathrm{H}, J=3.5, J=10.0), 5.60-5.69,5.64$ (dddd, $1 \mathrm{H}, J=7.5, J=7.5, J=14.5,, J=17.5$ ), 5.77 (dddd, $1 \mathrm{H}, J=7.5, J=7.5, J=14.5,, J=17.5$ ), 6.72 (brs, $1 \mathrm{H}), 7.10$ (t, $1 \mathrm{H}, J=7.0), 7.28-7.40$ (c, $4 \mathrm{H})$

${ }^{13}$ C NMR (101 MHz, CDCl3): $\delta$ 16.7, 17.0, 37.4, 37.7, 39.2, 39.4, 55.6, 61.4, 67.9, 68.8, 69.9, 71.1, 98.9, $117.2,117.4,118.9,124.3,129.4(3), 135.2,135.3,137.3,153.2,175.3,175.3$

IR (NaCl): 3339 (w), 2933 (w), 1745 (s), 1444 (w), 1222 (m), 1167 (m), $1053(\mathrm{~m}), 756(\mathrm{w}) \mathrm{cm}^{-1}$.

HRMS-ESI $(\mathrm{m} / \mathrm{z}):[\mathrm{M}+\mathrm{H}]^{+}$calcd for $\mathrm{C}_{26} \mathrm{H}_{36} \mathrm{NO} 9,506.2390$; found, 506.2386.

\section{[III_1_2_B]}

$\mathbf{t}_{\mathbf{R}}(\mathrm{LC} / \mathrm{MS}): 8.1 \mathrm{~min}$

${ }^{1}$ H NMR $\left(500 \mathrm{MHz}, \mathrm{CDCl}_{3}\right): \delta 1.13\left(\mathrm{~d}, \mathrm{~A} \_3 \mathrm{H}, J=7.0\right), 1.18$ (d, B_3H, $\left.J=6.5\right), 1.28\left(\mathrm{~d}, \mathrm{~A} \_3 \mathrm{H}, J=7.0\right)$, 1.30 (d, B_3H, $J=7.0), 1.93-3.04(\mathrm{c}, 7 \mathrm{H}), 3.40$ (s, 3H), 3.76-3.84 (c, 3H), 4.75 (d, A_1H, $J=1.5), 4.80$ (d, B_1H, $J=1.5$ ), $5.12\left(\mathrm{dd}, \mathrm{A} \_1 \mathrm{H}, J=4.0, J=10.0\right), 5.14$ (dd, B_1H, $J=4.0, J=10.0$ ), 5.17 (dd, B_1H, $J=1.5, J=4.0), 5.29(\mathrm{t}, 1 \mathrm{H}, J=10.0), 5.33\left(\mathrm{dd}, \mathrm{A} \_1 \mathrm{H}, J=1.5, J=4.0\right), 5.37-5.52(\mathrm{c}, 2 \mathrm{H}), 6.77$ (brs, 1H), 7.12 (t, 1H, $J=7.0), 7.28-7.40(\mathrm{c}, 4 \mathrm{H})$

${ }^{13}$ C NMR $\left(101 \mathrm{MHz}, \mathrm{CDCl}_{3}\right): \delta 17.2,19.2,37.2,39.2,40.6,41.2,55.6,61.5,67.3,68.9,70.0,70.8,99.2$, $119.0,124.4,126.4,127.4,128.7,129.5,131.3,137.7,153.1,174.6,175.7$

IR (NaCl): 3338 (w), 2936 (w), 1742 (s), 1541 (w), 1213 (m), 1136 (w), 1069 (m), 757 (w) cm .

HRMS-ESI $(\mathrm{m} / \mathrm{z}):[\mathrm{M}+\mathrm{H}]^{+}$calcd for $\mathrm{C}_{24} \mathrm{H}_{32} \mathrm{NO} 9,478.2077$; found, 478.2072.

* conformational mixture of $\mathrm{A}$ and $\mathrm{B}(\mathrm{A} / \mathrm{B}=5 / 2)$

Yields: $35 \mathrm{mg}$ of [III_1_2_M]-linked beads provided $9.8 \mathrm{mg}$ of pure [III_1_2_B]: $85 \%$ 


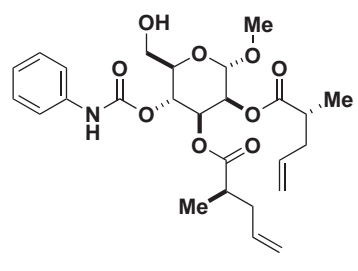

III $13 \mathrm{M}$

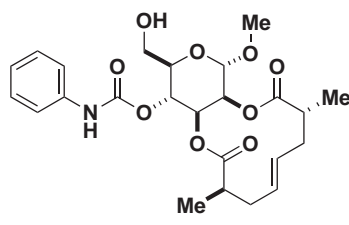

III 13 B

\section{[III_1_3_M]}

$\mathbf{t}_{\mathbf{R}}$ (LC/MS): $9.0 \mathrm{~min}$

${ }^{1}$ H NMR ( $500 \mathrm{MHz}, \mathrm{CDCl}_{3}$ ): $\delta 1.09$ (d, 3H, $J=7.0$ ), 1.20 (d, 3H, $J=6.5$ ), 2.09 (app quint, $1 \mathrm{H}, J=7.0, J$ $=7.5, J=14.5$ ), 2.24 (ddd, $1 \mathrm{H}, J=7.0, J=7.5, J=14.5$ ), 2.46 (app dt, 1H, J = 7.0, $J=14.0$ ), 2.47 (c, $2 \mathrm{H}$ ), 2.67 (dd, $1 \mathrm{H}, J=7.0, J=14.0$ ), 3.42 (s, 3H), 4.73 (brs, 1H), 4.85-4.96 (c, 2H), 5.06-5.13(c, 2H), $5.25(\mathrm{c}, 2 \mathrm{H}), 5.51(\mathrm{dd}, J=3.5, J=10.5), 5.62(\mathrm{c}, 1 \mathrm{H}), 5.78(\mathrm{c}, 1 \mathrm{H}), 6.66$ (brs, $1 \mathrm{H}), 7.11$ (t, $1 \mathrm{H}, J=7)$, 7.28-7.40 (c, 4H)

${ }^{13}$ C NMR $\left(101 \mathrm{MHz}, \mathrm{CDCl}_{3}\right): \delta 16.5,16.7,37.6,37.8,39.3,39.3,55.5,61.5,68.8,69.1,69.9,71.1,98.9$, 117.1, 117.5, 118.9, 124.3, 129.3, $129.4(2), 135.2(2), 137.2,153.1,175.2,175.2$ IR (NaCl): 3342(w), 2976(w), 2936(w), 1745 (s), 1602(w), 1445(w), 1070(m), 971(w), 758(w) $\mathrm{cm}^{-1}$. HRMS-ESI $(\mathrm{m} / \mathrm{z}):[\mathrm{M}+\mathrm{H}]^{+}$calcd for $\mathrm{C}_{26} \mathrm{H}_{36} \mathrm{NO}$, 506.2390; found, 506.2388 .

\section{[III_1_3_B]}

$\mathbf{t}_{\mathbf{R}}(\mathrm{LC} / \mathrm{MS}): 7.9 \mathrm{~min}$

${ }^{1} \mathbf{H}$ NMR(500 MHz, $\left.\mathrm{CDCl}_{3}\right): \delta 1.14$ (d, 3H, $\left.J=7.0\right), 1.18$ (d, 3H, $\left.J=7.0\right), 2.04-2.12(\mathrm{c}, 1 \mathrm{H}), 2.14-2.20$ (c, $1 \mathrm{H}), 2.30-2.48$ (c, 2H), 2.55-2.80 (c, 2H), 3.41 (s, 3H), 3.74-3.82 (c, 3H), 4.75 (d, A_1H, $J=1.5$ ), 4.80 (d, B_1H, $J=1.5), 5.12\left(\mathrm{dd}, \mathrm{A} \_1 \mathrm{H}, J=3.5, J=10.0\right), 5.15$ (dd, B_1H, $\left.J=3.5, J=10.0\right), 5.16$ (dd, B_1 $\mathrm{H}, J=1.5, J=3.5$ ), 5.29 (app t, $1 \mathrm{H}, J=10.0$ ), 5.32 (dd, A_1H, $J=1.5, J=3.5$ ), 5.48-5.54 (c, 2H), 6.67 (brs, 1H), 7.12 (t, 1H, $J=7.5), 7.35$ (t, 2H, $J=7.5), 7.41(\mathrm{br}, 2 \mathrm{H})$

${ }^{13} \mathbf{C}$ NMR $\left(101 \mathrm{MHz}, \mathrm{CDCl}_{3}\right): \delta 17.2,19.2,37.2,39.2,40.6,41.2,55.6,61.5,67.3,68.9,70.0,70.8,99.2$, $119.0,124.4,126.4,127.4,128.7,129.5,131.3,137.3,153.1,174.3,175.7$

IR ( $\mathrm{NaCl})$ : 3341 (w), 2970 (w), 1743 (s), 1445 ((w), 1209 (m), 1133 (m), 1066 (s), $758(\mathrm{w}) \mathrm{cm}^{-1}$.

HRMS-ESI $(\mathrm{m} / \mathrm{z}):[\mathrm{M}+\mathrm{H}]^{+}$calcd for $\mathrm{C}_{24} \mathrm{H}_{32} \mathrm{NO} 9,478.2077$; found, 478.2080 .

* conformational mixture of $\mathrm{A}$ and $\mathrm{B}(\mathrm{A} / \mathrm{B}=5 / 1)$

Yields: $50 \mathrm{mg}$ of [III_1_3_M]-linked beads provided $13.6 \mathrm{mg}$ of pure [III_1_3_B]: $92 \%$ 

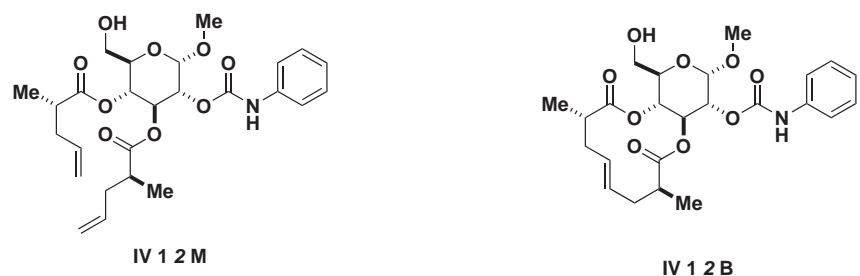

[IV_1_2_M]

$\mathbf{t}_{\mathbf{R}}$ (LC/MS): $9.3 \mathrm{~min}$

${ }^{1}$ H NMR $\left(500 \mathrm{MHz}, \mathrm{CDCl}_{3}\right): \delta 1.07(\mathrm{~d}, 3 \mathrm{H}, J=7.5), 1.21(\mathrm{~d}, 3 \mathrm{H}, J=7.0), 2.05-2.12(\mathrm{c}, 1 \mathrm{H}), 2.16$ (app quint, $1 \mathrm{H}, J=7.0), 2.30-2.43(\mathrm{c}, 3 \mathrm{H}), 2.44-2.58(\mathrm{c}, 2 \mathrm{H}), 3.43(\mathrm{~s}, 3 \mathrm{H}), 3.76-3.84(\mathrm{c}, 3 \mathrm{H}), 4.70(\mathrm{~d}, 1 \mathrm{H}, J=$ 2.0), 5.15 (dd, $J=3.5, J=10.0$ ), 5.01 (app t, $1 \mathrm{H}, J=10.0$ ), 5.43 (ddd, $1 \mathrm{H}, J=7.5, J=10.0, J=15.5$ ), 5.50 (ddd, 1H, $J=5.5, J=6.0, J=15.5$ ), 5.64 (app t, 1H, $J=10.0$ ), 6.71 (brs, 1H), 7.10 (m, 1H), 7.29$7.36(\mathrm{c}, 4 \mathrm{H})$

${ }^{13}$ C NMR (101 MHz, $\left.\mathrm{CDCl}_{3}\right): \delta 15.9,16.2,36.9,37.1,38.4,38.7,55.1,60.5,68.9,69.1,69.5,70.5,70.6$, $96.4,116.5,116.6,118.3,123.8,128.8,134.5,134.6,134.7,136.5,152.4,174.8,175.0$

IR (NaCl): 3337 (w), 2935 (w), 1743 (s), 1445 (w), 1203 (s), 1054 (s), 917 (w), 759 (w) cm . $^{-1}$

HRMS-ESI (m/z): $[\mathrm{M}+\mathrm{H}]^{+}$calcd for $\mathrm{C}_{26} \mathrm{H}_{36} \mathrm{NO}$, 506.2390; found, 506.2383.

\section{[IV_1_2_B]}

$\mathbf{t}_{\mathbf{R}}(\mathrm{LC} / \mathrm{MS}): 8.1 \mathrm{~min}$

${ }^{1} \mathbf{H} \operatorname{NMR}\left(500 \mathrm{MHz}, \mathrm{CDCl}_{3}\right): \delta 1.09$ (d, 3H, $\left.J=7.0\right), 1.15$ (d, 3H, $\left.J=6.5\right), 2.13-2.21(\mathrm{c}, 2 \mathrm{H}), 2.29-2.34$ (c, $1 \mathrm{H}), 2.47$ (ddd, 1H, $J=5.0, J=9.0, J=14.5), 2.71-286$ (c, 3H), 3.45 (s, 3H), 3.59 (dd, $1 \mathrm{H}, J=3.5, J$ $=13$ ), 3.73-3.80 (c, 1H), 4.98 (dd, 1H, $J=3.5, J=10.0), 4.98$ (t, 1H, $J=10.0), 5.44-5.52$ (c, 2H), 5.66 (app $\mathrm{t}, 1 \mathrm{H}, J=10.0), 6.78$ (brs, $1 \mathrm{H}), 7.08(\mathrm{t}, 1 \mathrm{H}, J=7.0), 7.28-7.40(\mathrm{c}, 4 \mathrm{H})$

${ }^{13}$ C NMR (101 MHz, $\left.\mathrm{CDCl}_{3}\right): \delta 14.7,16.3,36.2,37.1,40.5,40.6,55.7,61.1,68.9,69.0,69.8,71.6,97.7$, $118.8,124.1,126.7,129.4(2), 129.8$ (2), 137.5, 153.0, 174.4, 175.4 IR (NaCl): 3323(w), 2934(w), 1741(s), $1445(\mathrm{w}), 1212(\mathrm{~m}), 1087(\mathrm{~s}), 1042(\mathrm{~s}), 755(\mathrm{w}) \mathrm{cm}^{-1}$. HRMS-ESI $(\mathrm{m} / \mathrm{z}):[\mathrm{M}+\mathrm{H}]^{+}$calcd for $\mathrm{C}_{24} \mathrm{H}_{32} \mathrm{NO} 9,478.2077$; found, 478.2072.

Yields: 9.8 (20) mg of [IV_1_2_M]-linked beads provided 2.8 (5.5) mg of pure [IV_1_2_B]: $85 \%$ 

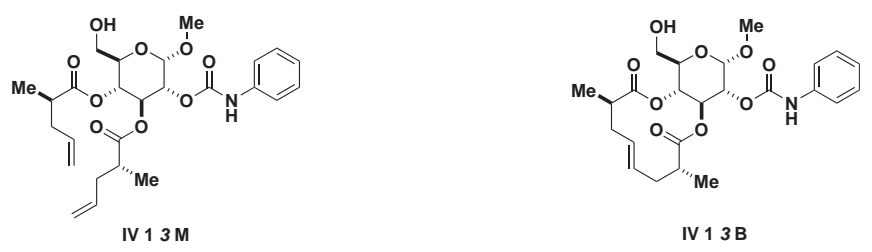

[IV_1_3_M]

$\mathbf{t}_{\mathbf{R}}$ (LC/MS): $8.2 \mathrm{~min}$

${ }^{1}$ H NMR $\left(500 \mathrm{MHz}, \mathrm{CDCl}_{3}\right): \delta 1.00$ (d, 3H, $\left.J=6.5\right), 1.05$ (d, 3H, $J=7.5$ ), 2.04-2.19 (c, 2H), 2.32-2.43 (c, $2 \mathrm{H}), 2.47-2.55$ (c, $2 \mathrm{H}), 3.44$ (s, $3 \mathrm{H}), 3.59$ (dd, $1 \mathrm{H}, J=4.5, J=12.5$ ), 3.71 (d, $1 \mathrm{H}, J=12.5$ ), 3.79 (ddd, $1 \mathrm{H}, J=2.5, J=4.5, J=10$ ), 4.86- 4.96 (c, 2H), 4.93 (dd, 1H, $J=3.5, J=10$ ), 5.02- 5.20 (c, $2 \mathrm{H}$ ), 5.04 (d, $1 \mathrm{H}, J=3.5$ ), 5.08 (app t, 1H, $J=10.0$ ), 5.62 (app t, 1H, $J=10.0$ ), 5,56- 5.76 (c, 2H), 6.82 (brs, 1H), 7.08 (t, $1 \mathrm{H}, J=7.0), 7.29-7.40(\mathrm{c}, 4 \mathrm{H})$

${ }^{13} \mathbf{C}$ NMR $\left(101 \mathrm{MHz}, \mathrm{CDCl}_{3}\right): \delta 16.7,16.8,37.5,37.7,39.2,39.3,55.7,61.3,68.9,69.7,69.7,71.8,97.5$, 117.2, 117.7, 118.8, 124.1, $129.4(3), 135.1,135.1,137.5,153.0,175.5,176.0$ IR ( $\mathrm{NaCl}): 3329$ (w), 2920 (w), 1744 (s), 1601 (w), 1540 (m), $1219(\mathrm{~m}), 1050(\mathrm{~m}), 755(\mathrm{w}) \mathrm{cm}^{-1}$. HRMS-ESI $(\mathrm{m} / \mathrm{z})$ : $[\mathrm{M}+\mathrm{H}]^{+}$calcd for $\mathrm{C}_{26} \mathrm{H}_{36} \mathrm{NO}$, 506.2390; found, 506.2386 .

\section{[IV_1_3_B]}

$\mathbf{t}_{\mathbf{R}}$ (LC/MS): $7.9 \mathrm{~min}$

H NMR (500 MHz, $\left.\mathrm{CDCl}_{3}\right): \delta 1.02(\mathrm{~d}, 3 \mathrm{H}, J=6.0), 1.16(\mathrm{~d}, 3 \mathrm{H}, J=6.5), 1.92(\mathrm{c}, 6 \mathrm{H}), 3.44(\mathrm{~s}, 3 \mathrm{H})$, 3.57 (dd, $1 \mathrm{H}, J=3.5, J=13), 3.69$ (d, $1 \mathrm{H}, J=13), 3.78$ (ddd, $1 \mathrm{H}, J=2, J=3.5, J=10.5), 4.92(\mathrm{dd}, 1 \mathrm{H}, J=$ 3.5, $J=10.5$ ), 5.01 (d, $J=3.0$ ), 5.09 (app t, 1H, J = 10.0), 5.37 (c, 2H), 5.63 (app t, 1H, $J=10.0$ ), 6.40 (brs, 1H), 6.79 (brs, 1H), 7.08 (t, 1H, $J=7.0), 7.28-7.40$ (c, 4H)

${ }^{13}$ C NMR $\left(101 \mathrm{MHz}, \mathrm{CDCl}_{3}\right): \delta 18.1,18.3,38.4,38.9,40.8,40.8,55.7,61.3,62.5,68.0,68.7,69.7,71.8$, 97.6, 118.89, 124.1, $129.4(2), 129.8$ (2), 137.5, 152.2, 176.1, 176.9

IR (NaCl): 3336 (w), 2926 (w), $1745(\mathrm{~s}), 1455(\mathrm{w}), 1222(\mathrm{~m}), 1170(\mathrm{~m}), 1088(\mathrm{w}), 754(\mathrm{w}) \mathrm{cm}^{-1}$.

HRMS-ESI (m/z): $[\mathrm{M}+\mathrm{H}]^{+}$calcd for $\mathrm{C}_{24} \mathrm{H}_{32} \mathrm{NO}$, 478.2077; found, 478.2072.

Yields: $36 \mathrm{mg}$ of [IV_1_3_M]-linked beads provided $10.7 \mathrm{mg}$ of pure [IV_1_3_B]: $90 \%$ 

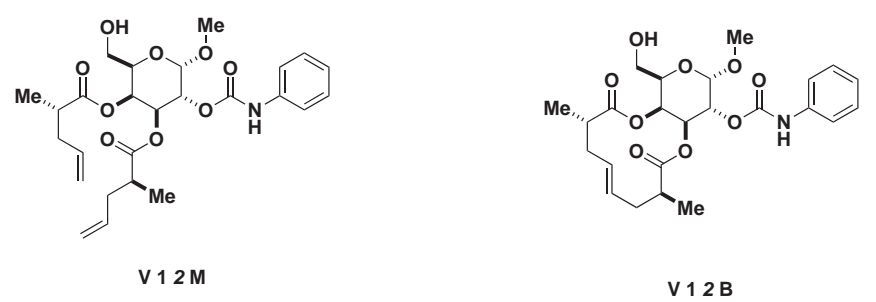

[V_1_2_M]

$\mathbf{t}_{\mathbf{R}}$ (LC/MS): $11.8 \mathrm{~min}$

${ }^{1}$ H NMR (500 MHz, $\left.\mathrm{CDCl}_{3}\right): \delta 1.06(\mathrm{~d}, 3 \mathrm{H}, J=7.5), 1.24(\mathrm{~d}, 3 \mathrm{H}, J=7.5), 2.08$ (app quint, $\left.1 \mathrm{H}, J=7.0\right)$, $2.20-2.51(\mathrm{c}, 4 \mathrm{H}), 2.70(\mathrm{c}, 1 \mathrm{H}), 3.45(\mathrm{~s}, 3 \mathrm{H}), 3.48(\mathrm{dd}, 1 \mathrm{H}, J=7.5, J=11.5), 3.66(\mathrm{dd}, 1 \mathrm{H}, J=6.5, J=$ 11.5 ), 4.09 (app t, $1 \mathrm{H}, J=7$ ), 4.95- 5.14 (c, 4H), 5.05 (d, 1H, $J=3.5), 5.28$ (dd, $1 \mathrm{H}, J=3.5, J=10.5$ ), $5.42(\mathrm{~d}, 1 \mathrm{H}, J=3.5), 5.48(\mathrm{dd}, 1 \mathrm{H}, J=3.5, J=10.5), 5.61-5.82$ (c, $2 \mathrm{H}), 6.78$ (brs, $1 \mathrm{H}), 7.09$ (app t, $1 \mathrm{H}, J$ $=7), 7.30-7.38(\mathrm{c}, 4 \mathrm{H})$

${ }^{13}$ C NMR (101 MHz, $\left.\mathrm{CDCl}_{3}\right): \delta 16.7,17.3,37.4,37.8,39.4,39.6,55.7,60.9,67.8,69.2,69.3$ (2), 97.9, 117.3, 117.7, 118.8, 124.1, 129.4 (3), 135.2, 135.3, 137.5, 152.5, 175.1, 176.6

IR (NaCl): 3330 (w), 2974 (w), 2934 (w), 1743 (s), 1600 (w), 1445 (w), 1221 (m), 920 (w), 757 (w) cm . HRMS-ESI $(\mathrm{m} / \mathrm{z}):[\mathrm{M}+\mathrm{H}]^{+}$calcd for $\mathrm{C}_{26} \mathrm{H}_{36} \mathrm{NO}$, 506.2390; found, 506.2386.

\section{[V_1_2_B]}

$\mathbf{t}_{\mathbf{R}}(\mathrm{LC} / \mathrm{MS}): 8.1 \mathrm{~min}$

${ }^{1} \mathbf{H} \mathbf{N M R}\left(500 \mathrm{MHz}, \mathrm{CDCl}_{3}\right): \delta 1.08(\mathrm{~d}, 3 \mathrm{H}, J=7.0), 1.19$ (d, 3H, $\left.J=6.5\right), 1.98-2.22(\mathrm{c}, 3 \mathrm{H}), 2.31$ (br d, $1 \mathrm{H}, J=12), 2.50-2.60(\mathrm{c}, 2 \mathrm{H}), 2.75(\mathrm{c}, 1 \mathrm{H}), 3.40-3.45(\mathrm{c}, 1 \mathrm{H}), 3.46(\mathrm{~s}, 3 \mathrm{H}), 3.57-3.63$ (c, 1H), 4.08 (appt $, 1 \mathrm{H}, J=7), 5.02(\mathrm{~d}, 1 \mathrm{H}, J=4.0), 5.13(\mathrm{dd}, J=3.5, J=11.0), 5.24(\mathrm{ddd}, 1 \mathrm{H}, J=3, J=12, J=13), 5.44$ (ddd, $1 \mathrm{H}, J=4.0, J=11.0), 5.59$ (ddd, $1 \mathrm{H}, J=3, J=12, J=13$ ), 5.62 (d, $1 \mathrm{H}, J=3.5$ ), 6.79 (brs, $1 \mathrm{H}$ ), $7.10(\mathrm{t}, 1 \mathrm{H}, J=7.0), 7.29-7.40(\mathrm{c}, 4 \mathrm{H})$

${ }^{13}$ C NMR $\left(101 \mathrm{MHz}, \mathrm{CDCl}_{3}\right): \delta 17.5,19.1,37.1,39.1,40.9,41.3,55.8,60.5,68.6,68.7,68.9,69.0,98.1$, $118.9,124.1,128.1,128.8,129.4(2), 131.3,137.6,152.4,174.7,177.7$

IR (NaCl): 3330 (w), 2935 (w), 2845 (w), 1741 (s), 1600 (w), 1445 (w), $1213(\mathrm{~m}), 756(\mathrm{w}) \mathrm{cm}^{-1}$.

HRMS-ESI $(\mathrm{m} / \mathrm{z}):[\mathrm{M}+\mathrm{H}]^{+}$calcd for $\mathrm{C}_{2} 4 \mathrm{H}_{32} \mathrm{NO} 9,478.2077$; found, 478.2072.

Yields: $40 \mathrm{mg}$ of [V_1_2_M]-linked beads provided $11.2 \mathrm{mg}$ of pure [V_1_2_B]: $85 \%$ 

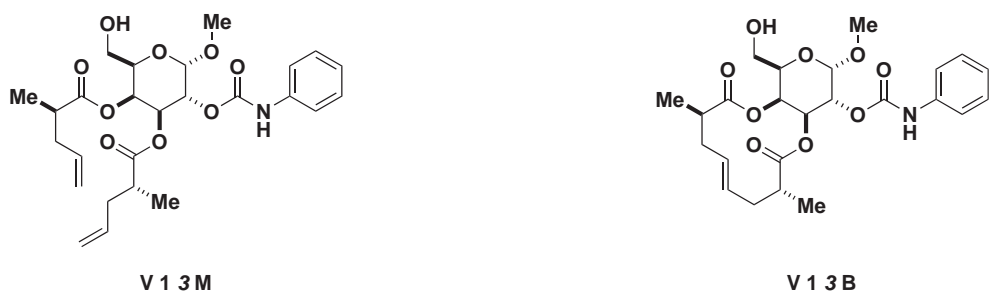

[V_1_3_M]

$\mathbf{t}_{\mathbf{R}}(\mathrm{LC} / \mathrm{MS}): 11.4 \mathrm{~min}$

${ }^{1}$ H NMR $\left(500 \mathrm{MHz}, \mathrm{CDCl}_{3}\right): \delta 1.08(\mathrm{~d}, 3 \mathrm{H}, J=6.5), 1.24(\mathrm{~d}, 3 \mathrm{H}, J=6.5), 2.10$ (app quint, $\left.1 \mathrm{H}, J=7.0\right)$, $2.20-2.51(\mathrm{c}, 4 \mathrm{H}), 2.69(\mathrm{c}, 1 \mathrm{H}), 3.45(\mathrm{~s}, 3 \mathrm{H}), 3.49(\mathrm{dd}, 1 \mathrm{H}, J=7.0, J=11.5), 3.66(\mathrm{dd}, 1 \mathrm{H}, J=7.0, J=$ 11.5 ), 4.10 (app t, $1 \mathrm{H}, J=7.0), 4.89-5.14$ (c, $4 \mathrm{H}), 5.07$ (d, $1 \mathrm{H}, J=3.5), 5.27$ (dd, $1 \mathrm{H}, J=3.5, J=11$ ), $5.43(\mathrm{~d}, 1 \mathrm{H}, J=3.5), 5.48(\mathrm{dd}, 1 \mathrm{H}, J=3.5, J=11), 5.58-5.82(\mathrm{c}, 2 \mathrm{H}), 6.78$ (brs, $1 \mathrm{H}), 7.09$ (app t, $1 \mathrm{H}, J$ $=7), 7.30-7.38(\mathrm{c}, 4 \mathrm{H})$

${ }^{13}$ C NMR (101 MHz, $\left.\mathrm{CDCl}_{3}\right): \delta 16.2,16.9,37.4,37.5,39.0,39.4,55.5,60.7,67.5,67.8,69.0,69.0,97.7$, $116.9,117.3,118.5,123.8,129.4(2), 132.1,134.9,135.0,137.3,152.2,174.9,176.2$

IR (NaCl): 3334 (w), 2976 (w), 2937 (w), 1743 (s), 1540 (w), 1445 (w), 1221 (m), 756 (w) cm .

HRMS-ESI $(\mathrm{m} / \mathrm{z}):[\mathrm{M}+\mathrm{H}]^{+}$calcd for $\mathrm{C}_{26} 6 \mathrm{H}_{36} \mathrm{NO9}, 506.2390$; found, 506.2386.

\section{[V_1_3_B]}

$\mathbf{t}_{\mathbf{R}}(\mathrm{LC} / \mathrm{MS}): 7.9 \mathrm{~min}$

${ }^{1} \mathbf{H} \mathbf{N M R}\left(500 \mathrm{MHz}, \mathrm{CDCl}_{3}\right): \delta 1.14\left(\mathrm{~d}, \mathrm{~A} \_3 \mathrm{H}, J=7.0\right), 1.18\left(\mathrm{~d}, \mathrm{~B} \_3 \mathrm{H}, J=7.0\right), 1.32\left(\mathrm{~d}, \mathrm{~A} \_3 \mathrm{H}, J=7.0\right)$, 1.34 (d, B_3H, $J=7.0), 1.92-3.05(\mathrm{c}, 6 \mathrm{H}), 3.36-3.46$ (c, 1H), $3.43(\mathrm{~s}, 3 \mathrm{H}), 3.55-3.62(\mathrm{c}, 1 \mathrm{H}), 4.05-4.08$ (c, $1 \mathrm{H}), 5.03\left(\mathrm{~d}, \mathrm{~A} \_1 \mathrm{H}, J=3.5\right), 5.04\left(\mathrm{~d}, \mathrm{~B} \_1 \mathrm{H}, J=3.5\right), 5.12\left(\mathrm{dd}, \mathrm{A} \_1 \mathrm{H}, J=3.5, J=10.5\right), 5.16\left(\mathrm{dd}, \mathrm{B} \_1 \mathrm{H}\right.$, $J=3.5, J=10.5), 5.30\left(\mathrm{dd}, \mathrm{A} \_1 \mathrm{H}, J=3.5, J=10.5\right), 5.31\left(\mathrm{dd}, \mathrm{B} \_1 \mathrm{H}, J=3.5, J=10.5\right), 5.40$ (br d, A $1 \mathrm{H}, J=3.5), 5.36-5.59$ (c, 2H and B_1H), 6.80 (brs, $1 \mathrm{H}), 7.10$ (t, $1 \mathrm{H}, J=7.0), 7.32-7.42$ (c, 4H)

${ }^{13}$ C NMR $\left(101 \mathrm{MHz}, \mathrm{CDCl}_{3}\right): \delta 15.8,16.7,36.4,37.2,38.8,41.3,55.7,60.4,68.7,68.9,69.1$ (2), 97.8, $118.8,124.1,127.4,128.9,129.4(2), 130.2,137.6,152.5,175.3,176.4$ IR (NaCl): 3330 (w), 2935 (w), 2845 (w), 1741 (s), 1600 (w), 1445 (w), 1077 (m), 756 (w) cm . HRMS-ESI $(\mathrm{m} / \mathrm{z}):[\mathrm{M}+\mathrm{H}]^{+}$calcd for $\mathrm{C}_{24} \mathrm{H}_{32} \mathrm{NO}$, 478.2077; found, 478.2072.

* conformational mixture of $\mathrm{A}$ and $\mathrm{B}(\mathrm{A} / \mathrm{B}=2 / 1)$

Yields: 25 (8.0) mg of [V_1_3_M]-linked beads provided 7.8 (2.5) mg of pure [V_1_3_B]: $95 \%$ 

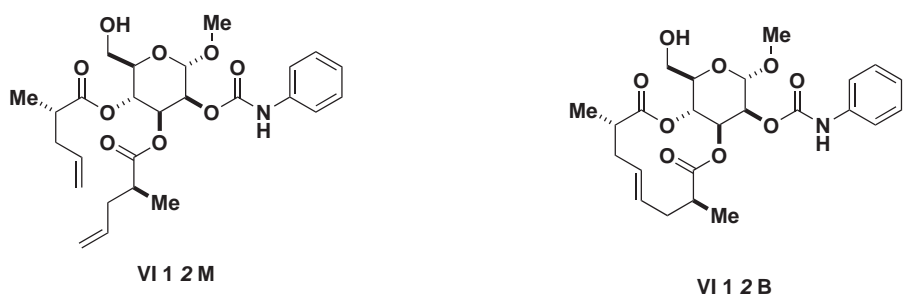

\section{[VI_1_2 M]}

$\mathbf{t}_{\mathbf{R}}(\mathrm{LC} / \mathrm{MS}): 8.1 \mathrm{~min}$

${ }^{1} \mathbf{H} \operatorname{NMR}\left(500 \mathrm{MHz}, \mathrm{CDCl}_{3}\right): \delta 1.09(\mathrm{~d}, 3 \mathrm{H}, J=6.5), 1.15(\mathrm{~d}, 3 \mathrm{H}, J=6.5), 2.06$ (app quint, $J=7, J=7, J$ =14), 2.12 (app quint, $J=7, J=7, J=14$ ), 2.30 (c, 3H), 2.48 (app sext, $J=7, J=7, J=7$ ), 2.66 (br s, 1), $3.36(\mathrm{~s}, 3 \mathrm{H}), 3.58(\mathrm{dd}, 1 \mathrm{H}, J=2.5, J=12.5), 3.67-3.73(\mathrm{c}, 2 \mathrm{H}), 4.77(\mathrm{~d}, 1 \mathrm{H}, J=1.5), 4.89-5.05(\mathrm{c}, 4 \mathrm{H})$, $5.20(\mathrm{dd}, 1 \mathrm{H}, J=1.5, J=3.5), 5.28($ app t, $1 \mathrm{H}, J=10.0), 5.41$ (dd, $1 \mathrm{H}, J=3.5, J=10.0), 5.56-5.72$ (c, $2 \mathrm{H}), 7.03(\mathrm{t}, 1 \mathrm{H}, J=7.5), 7.32(\mathrm{t}, 1 \mathrm{H}, J=7.5), 7.39(\mathrm{~d}, 2 \mathrm{H}, J=7.5)$

${ }^{13}$ C NMR (101 MHz, $\left.\mathrm{CDCl}_{3}\right): \delta 16.4,16.9,37.6,37.8,39.1,39.4,55.6,66.5,69.1,70.4,70.7,99.1$, 117.3, 117.6, 117.7, 118.8, 123.9 (2), 129.3, 135.1, 135.2, 135.4, 137.7, 152.3, 175.0, 176.5

IR (NaCl): 3516(w), $3325(\mathrm{w}), 2977(\mathrm{w}), 2937(\mathrm{w}), 1744(\mathrm{~s}), 1601(\mathrm{w}), 1542(\mathrm{w}), 1221(\mathrm{~m}), 1137$ (m), $1096(\mathrm{~m}), 758(\mathrm{w}) \mathrm{cm}^{-1}$.

HRMS-ESI $(\mathrm{m} / \mathrm{z}):[\mathrm{M}+\mathrm{H}]^{+}$calcd for $\mathrm{C}_{26} \mathrm{H}_{36} \mathrm{NO9}$, 506.2390; found, 506.2394.

\section{[VI_1_2_B]}

$\mathbf{t}_{\mathbf{R}}(\mathrm{LC} / \mathrm{MS}): 7.9 \mathrm{~min}$

H NMR(500 MHz, $\left.\mathrm{CDCl}_{3}\right): \delta 1.05(\mathrm{~d}, 3 \mathrm{H}, J=7.5), 1.17(\mathrm{~d}, 3 \mathrm{H}, J=7.0), 2.06-2.11(\mathrm{~m}, 1 \mathrm{H}), 2.14-2.21$ (m, 1H), 2.33-2.42 (c, 2H), 2.71 (br s, 1H), 2.74-2.86 (c, 2H), $3.42(\mathrm{~s}, 3 \mathrm{H}), 3.62(\mathrm{br} \mathrm{d}, 1 \mathrm{H}, J=11), 3.73$ (br d, 1H, $J=11$ ), 3.78 (br d, $1 \mathrm{H}, J=10.5), 4.81$ (d, 1H, $J=2.0), 5.25$ (dd, $J=2.0, J=3.5$ ), 5.31 (app t, $1 \mathrm{H}, J=10.5), 5.40-5.49$ (c, 2H), 5.45 (dd, 1H, $J=3.5, J=10.5), 7.07$ (t, 1H, $J=7), 7.14$ (brs, $1 \mathrm{H}), 7.31$ $(\mathrm{t}, 2 \mathrm{H}, J=7), 7.41(\mathrm{~d}, 2 \mathrm{H}, J=7)$

${ }^{13} \mathbf{C}$ NMR $\left(101 \mathrm{MHz}, \mathrm{CDCl}_{3}\right): \delta 15.1\left(\mathrm{CH}_{3}\right), 15.6\left(\mathrm{CH}_{3}\right), 36.8\left(\mathbf{C H}_{2}\right), 36.8\left(\mathbf{C H}_{2}\right), 40.5(\mathbf{C H}), 40.6(\mathbf{C H})$, 55.6(CH), 61.3( $\left(\mathrm{CH}_{2}\right)$, 66.1 $(\mathbf{C H}), 68.6(\mathbf{C H}), 70.5(\mathbf{C H}), 70.7(\mathbf{C H}), 99.3(\mathbf{C H}), 118.8(\mathbf{C H}), 123.9(\mathbf{C H})$, 127.8(CH), $128.2(\mathbf{C H}), 128.5(\mathbf{C H}), 129.3\left(2 \_\mathbf{C H}\right), 137.7(\mathbf{C}), 152.4(\mathbf{C O}), 173.7(\mathbf{C O}), 175.3(\mathbf{C O})$ IR (NaCl): 3333 (w), 2935 (w), 2845 (w), 1743 (s), 1600 (w), 1445 (w), 1213 (m), 758 (w) $\mathrm{cm}^{-1}$.

HRMS-ESI $(\mathrm{m} / \mathrm{z}):[\mathrm{M}+\mathrm{H}]^{+}$calcd for $\mathrm{C}_{2} 4 \mathrm{H} 32 \mathrm{NO}$, 478.2077; found, 478.2071.

Yields: 24 mg of [VI_1_2_M]-linked beads provided 6.7 mg of pure [VI_1_2_B]: $85 \%$ 

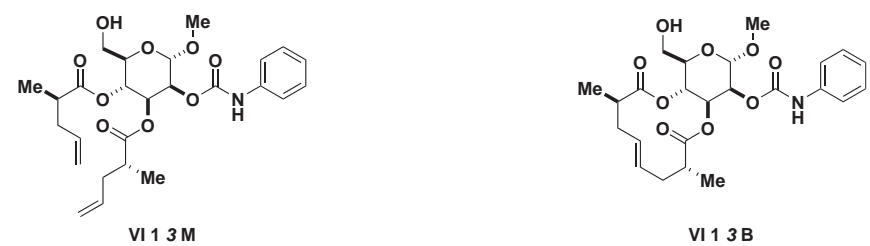

$\mathbf{t}_{\mathbf{R}}(\mathrm{LC} / \mathrm{MS}): 9.0 \mathrm{~min}$

${ }^{1}$ H NMR(500 MHz, $\left.\mathrm{CDCl}_{3}\right): \delta 1.09(\mathrm{~d}, 3 \mathrm{H}, J=6.5), 1.16(\mathrm{~d}, 3 \mathrm{H}, J=7.0), 2.09(\mathrm{ddd}, J=5, J=7, J=14)$, 2.16 (app quint, $J=7, J=7, J=14$ ), 2.34-2.58 (c, 5H), 3.43 (s, 3H), 3.61 (br d, 1H, $J=13$ ), 3.71-3.78 (c, $2 \mathrm{H}), 4.84(\mathrm{~d}, 1 \mathrm{H}, J=1.5), 4.96-5.02(\mathrm{c}, 4 \mathrm{H}), 5.29(\mathrm{dd}, 1 \mathrm{H}, J=1.5, J=3.5), 5.33$ (app t, $1 \mathrm{H}, J=10.0)$, 5.47 (dd, 1H, $J=3.5, J=10$ ), 5.59-5.74 (c, 2H), 6.90 (br s, 1H), 7.09 (t, 1H, $J=7.5), 7.32$ (t, 1H, $J=$ 7.5), $7.40(\mathrm{~d}, 2 \mathrm{H}, J=7.5)$

${ }^{13}$ C NMR (101 MHz, $\left.\mathrm{CDCl}_{3}\right): \delta 15.9,16.3,36.8,36.9,38.7,38.9,55.0,60.7,65.7,68.4,69.7,70.1,98.5$, $116.6,117.3,118.5,123.8,129.1(3), 134.96,135.0,137.2,152.2,174.9,176.2$

IR (NaCl): 3315 (w), 2937 (w), 1739 (s), 1601 (w), 1542(w), 1218 (m), 1096 (m), 974 (w), 757 (w) cm . HRMS-ESI $(\mathrm{m} / \mathrm{z}):[\mathrm{M}+\mathrm{H}]^{+}$calcd for $\mathrm{C}_{26} \mathrm{H}_{36} \mathrm{NO}$, 506.2390; found, 506.2394.

\section{[VI_1_3_B]}

$\mathbf{t}_{\mathbf{R}}(\mathrm{LC} / \mathrm{MS}): 8.3 \mathrm{~min}$

${ }^{1} \mathbf{H} \operatorname{NMR}\left(500 \mathrm{MHz}, \mathrm{CDCl}_{3}\right): \delta 1.155(\mathrm{~d}, 3 \mathrm{H}, J=6.5), 1.16(\mathrm{~d}, 3 \mathrm{H}, J=6.5), 2.09-2.16(\mathrm{c}, 3 \mathrm{H}), 2.35-2.52$ (c, 4H), 3.43 (s, 3H), 3.60 (ddd, $1 \mathrm{H}, J=4, J=5, J=13), 3.71$ (ddd, $1 \mathrm{H}, J=2.5, J=9.5, J=13$ ), 3.78 (ddd, $1 \mathrm{H}, J=2.5, J=4.5, J=10.5), 4.85$ (d, 1H, $J=1.5), 5.19$ (dd, $J=1.5, J=3.5), 5.30$ (app t, $1 \mathrm{H}, J=$ 10.5), 5.30-5.40 (c, 2H), 5.52 (dd, $1 \mathrm{H}, J=3.5, J=10.5$ ), 6.85 (brs, $1 \mathrm{H}), 7.09$ (t, $1 \mathrm{H}, J=7), 7.33$ (t, $2 \mathrm{H}, J$ $=7), 7.41(\mathrm{~d}, 2 \mathrm{H}, J=7)$

${ }^{13}$ C NMR $\left(101 \mathrm{MHz}, \mathrm{CDCl}_{3}\right): \delta 17.519 .1,37,1,39.1,40.9,41.3,55.8,60.5,68.6,68.7,68.9,69.0,98.1$, $118.9,124.1,128.8,129.4(3), 131.3,137.6,152.4,174.7,177.7$

IR (NaCl): 2931(w), 1743 (s), 1545 (w), 1444(w), 1219 (m), $1176(\mathrm{~m}), 1094(\mathrm{~m}), 967$ (w), 754(w) $\mathrm{cm}^{-1}$. HRMS-ESI $(\mathrm{m} / \mathrm{z}):[\mathrm{M}+\mathrm{H}]^{+}$calcd for $\mathrm{C}_{24} \mathrm{H}_{32} \mathrm{NO} 9,478.2077$; found, 478.2067

Yields: 40 mg of [VI_1_3_M]-linked beads provided $11.0 \mathrm{mg}$ of pure [VI_1_3_B]: 84 \% 

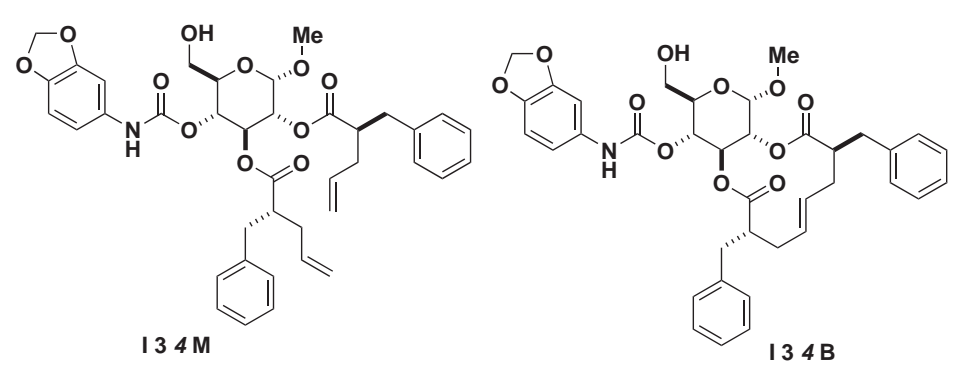

\section{[I_3_4_M]}

$\mathbf{t}_{\mathbf{R}}(\mathrm{LC} / \mathrm{MS}): 10.6 \mathrm{~min}$.

${ }^{1}$ H NMR(500 MHz, $\left.\mathrm{CDCl}_{3}\right): \delta$ 2.08-2.28 (c, 3H), 2.58-2.71 (c, 3H), 2.86-3.00 (c, 3H), 3.34 (s, 3H), 3.69$3.80(\mathrm{c}, 3 \mathrm{H}), 4.82(\mathrm{dd}, 1 \mathrm{H}, J=3.5, J=10), 4.93(\operatorname{app~t}, J=10), 5.01(\mathrm{~d}, 1 \mathrm{H}, J=3.5), 4.80-5.06(\mathrm{c}, 4 \mathrm{H})$, 5.60-5.73 (c, 2H), 5.93 (s, 2H), 6.87 (br s, 1H), 6.65-7.29 (c, $13 \mathrm{H})$

${ }^{13} \mathbf{C}$ NMR $\left(101 \mathrm{MHz}, \mathrm{CDCl}_{3}\right): \delta 34.2\left(\mathbf{C H}_{2}\right), 34.8\left(\mathbf{C H}_{2}\right), 35.9\left(\mathbf{C H}_{2}\right), 36.1\left(\mathbf{C H}_{2}\right), 45.7(\mathbf{C H}), 54.8(\mathbf{C H})$, $60.3\left(\mathrm{CH}_{2}\right), 68.9(\mathbf{C H}), 69.1(\mathbf{C H}), 69.4(\mathbf{C H}), 70.9(\mathbf{C H}), 96.1(\mathbf{C H}), 100.8(\mathbf{C}), 101.2(\mathbf{C}), 107.6(\mathbf{C H})$, $111.5(\mathbf{C H}), 116.9\left(\mathbf{C H}_{2}\right), 116.9\left(\mathbf{C H}_{2}\right), 125.9\left(3 \_\mathbf{C H}\right), 127.5(\mathbf{C H}), 127.7(\mathbf{C H}), 127.9\left(3 \_\mathbf{C H}\right), 128.4$ $(\mathbf{C H}), 128.5$ (3_CH), 130.7 (C), $134.0(\mathbf{C}), 134.0(\mathbf{C}), 138.2(\mathbf{C H}), 143.7(\mathbf{C}), 147.5(\mathbf{C}), 152.5(\mathbf{C O})$, $173.4(\mathbf{C O}), 173.7(\mathbf{C O})$

IR (NaCl): 3344 (w), 2924 (w), 1741 (s), 1549 (w), 1213 (m), 1162 (m), 1039 (m), 920 (w), $701(w) \mathrm{cm}^{-1}$. HRMS-ESI $(\mathrm{m} / \mathrm{z}):[\mathrm{M}+\mathrm{H}]^{+}$calcd for $\mathrm{C}_{39} \mathrm{H}_{44} \mathrm{NO}_{11}, 702.2914$; found, 702.2920 .

\section{[I_3_4_B]}

$\mathbf{t}_{\mathbf{R}}$ (LC/MS): $9.8 \mathrm{~min}$.

${ }^{1}$ H NMR(500 MHz, $\left.\mathrm{CDCl}_{3}\right): \delta$ 2.20-2.28 (c, 2H), $2.43(\mathrm{~m}, 1 \mathrm{H}), 2.69(\mathrm{dd}, 1 \mathrm{H}, J=3.5, J=14.0), 2.95-$ $3.06(\mathrm{c}, 3 \mathrm{H}), 3.11(\mathrm{dd}, 1 \mathrm{H}, J=4.5, J=14.0), 3.25$ (s, 3H), 3.69-3.80 (c, 3H), 4.84 (d, 1H, $J=3.5), 4.86$ (dd, 1H, $J=3.5, J=10), 4.95$ (app t, $J=10), 5.54$ (c, 2H), 5.71 (app t, 1H, $J=10), 5.92$ (s, 2H), 6.79 (br s, 1H), 7.00-7.29 (c, $13 \mathrm{H})$

${ }^{13} \mathbf{C}$ NMR $\left(101 \mathrm{MHz}, \mathrm{CDCl}_{3}\right): \delta 34.1\left(\mathbf{C H}_{2}\right) 35.3\left(\mathbf{C H}_{2}\right), 35.4\left(\mathbf{C H}_{2}\right), 36.9\left(\mathbf{C H}_{2}\right), 47.8(\mathbf{C H}), 48.1(\mathbf{C H})$, 55.6 $(\mathbf{C H}), 61.2\left(\mathrm{CH}_{2}\right), 69.4(\mathbf{C H}), 69.9(\mathbf{C H}), 70.1(\mathbf{C H}), 71.4(\mathbf{C H}), 97.1(\mathbf{C H}), 101.6\left(\mathrm{CH}_{2}\right), 101.9$ $(\mathbf{C H}), 108.4(\mathbf{C H}), 112.2(\mathbf{C H}), 126.5(\mathbf{C H}), 126.5(\mathbf{C H}), 126.7(\mathbf{C H}), 126.8(\mathbf{C H}), 127.4(\mathbf{C H}), 128.6$ $(\mathbf{C H}), 128.7(\mathbf{C H}), 128.7(\mathbf{C H}), 128.8(\mathbf{C H}), 128.9(\mathbf{C H}), 129.1(\mathbf{C H}), 129.2(\mathbf{C H}), 131.5(\mathbf{C}), 139.0(\mathbf{C})$, $139.5(\mathbf{C}), 144.4(\mathbf{C}), 148.3(\mathbf{C}), 153.1(\mathbf{C O}), 173.4(\mathbf{C O}), 173.8(\mathbf{C O})$

IR $(\mathrm{NaCl}): 3341(\mathrm{w}), 2924(\mathrm{w}), 1736(\mathrm{~s}), 1503(\mathrm{w}), 1243(\mathrm{~m}), 1038(\mathrm{~s}), 700(\mathrm{w}) \mathrm{cm}^{-1}$.

HRMS-ESI $(\mathrm{m} / \mathrm{z})$ : $[\mathrm{M}+\mathrm{H}]^{+}$calcd for $\mathrm{C}_{37} \mathrm{H}_{40} \mathrm{NO}_{11}, 674.2601$; found, 674.2601 .

Yields: $52 \mathrm{mg}$ of [I_3_4_M]-linked beads provided $20.3 \mathrm{mg}$ of pure [I_3_4_B]: $90 \%$ 

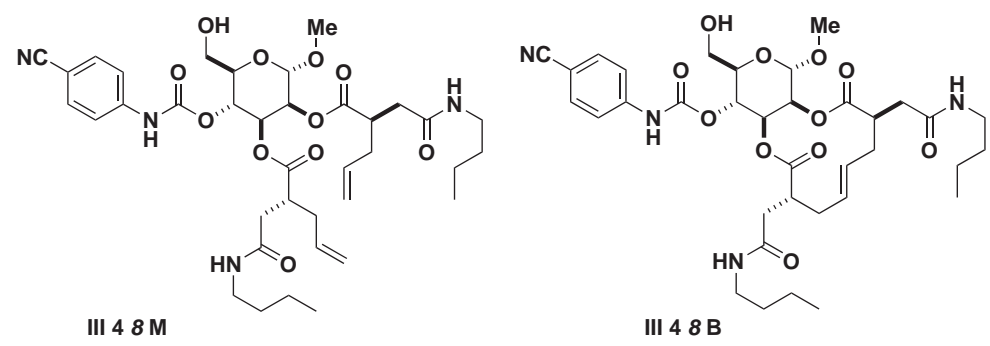

\section{[III_4_8_M]}

$\mathbf{t}_{\mathbf{R}}(\mathrm{LC} / \mathrm{MS}): 8.9 \mathrm{~min}$.

${ }^{1} \mathbf{H}$ NMR(500 MHz, $\left.\mathrm{CDCl}_{3}\right): \delta$ 0.82-0.93 (c, 6H), 1.18-1.31 (c, 4H), 1.37-1,49 (c, 4H), 2.14-2.82 (c, 6H), 3.00-3.25 (c, 4H), $3.78(\mathrm{~s}, 3 \mathrm{H}), 3.76-3.89(\mathrm{c}, 3 \mathrm{H}), 4.73$ (d, A_1H, $J=1.5), 4.71$ (d, B_1H, $J=1.5), 5.00-$ $5.22(\mathrm{c}, 4 \mathrm{H}), 5.25\left(\mathrm{dd}, \mathrm{A} \_\mathrm{B} \_1 \mathrm{H}, J=1,5, J=4.0\right), 5.42\left(\mathrm{dd}, \mathrm{B} \_1 \mathrm{H}, J=4.0 J=10\right), 5.47$ (dd, A_1H, $J=$ $4.0 J=10), 5.58$ (app t, $1 \mathrm{H}, J=10), 5.54-5.83$ (c, 2H), 7.45-7.59 (c, $4 \mathrm{H}$ )

${ }^{13}$ C NMR (101 MHz, $\left.\mathrm{CDCl}_{3}\right): \delta 13.9,14.0,20.3,20.3,31.7,31.8,35.4,36.1,37.1,37.1,39.6,39.7,41.3$, 42.0, 55.6, 61.4, 68.2, 69.4, 70.2, 71.1, 98.8, 106.8, 117.8, 118.3, 118.8, 119.0, 133.5, 134.7, 134.8, $142.1,153.3,170.8,171.1,173.4,173.5$

IR (NaCl): 3308 (m), 2958 (m), 2225 (s), 1744 (s), 1649 (m), 1534 (m), 1219 (s), 1064 (m), 842 (w) cm . HRMS-ESI $(\mathrm{m} / \mathrm{z})$ : $[\mathrm{M}+\mathrm{H}]^{+}$calcd for $\mathrm{C}_{37} \mathrm{H}_{53} \mathrm{~N}_{4} \mathrm{O} 9,729.3711$; found, 729.3704 .

\section{[III_4_8_B]}

$\mathbf{t}_{\mathbf{R}}$ (LC/MS): $8.4 \mathrm{~min}$.

${ }^{1} \mathbf{H}$ NMR(500 MHz, $\left.\mathrm{CDCl}_{3}\right): \delta$ 0.84-0.99 (c, 6H), 1.21-1.55 (c, 8H), 1.92-2.82 (c, 6H), 3.00-3.25 (c, 4H), $3.41(\mathrm{~s}, 3 \mathrm{H}), 3.76-3.89(\mathrm{c}, 3 \mathrm{H}), 4.71\left(\mathrm{~d}, \mathrm{~A} \_1 \mathrm{H}, J=1.5\right), 5.14\left(\mathrm{app} \mathrm{t}, \mathrm{A} \_1 \mathrm{H}, J=10\right), 5.23\left(\mathrm{dd}, \mathrm{A} \_1 \mathrm{H}, J=\right.$ $4.0, J=10), 5.41$ (dd, A_1H, $J=1.5 J=4.0), 5.54$ (c, 2H), 6.91 (br s, 1H), 7.45-7.59 (c, $4 \mathrm{H})$

${ }^{13}$ C NMR (101 MHz, $\left.\mathrm{CDCl}_{3}\right): \delta 12.7$ (2), 19.0 (2), 30.4, 30.6, 33.2, 34.3, 35.4, 37.6, 38.3, 38.6, 41.5, $42.4,54.2,60.4,67.5,68.3,69.0,69.6,97.7,105.3,117.6,117.9,124.5,127.2,130.1,132.2$ (2), 132.5, $134.7,141.3,152.5,168.9,169.1,171.0,173.4$ IR (NaCl): 3315 (s), 2930 (m), 2225 (s), 1742 (s), 1647 (s), 1597 (s), 1215 (m), 1066 (m), 700 (w) cm . HRMS-ESI $(\mathrm{m} / \mathrm{z})$ : $[\mathrm{M}+\mathrm{H}]^{+}$calcd for $\mathrm{C}_{35} \mathrm{H}_{49} \mathrm{~N} 4 \mathrm{O} 9,701.3398$; found, 701.3391.

* A is major conformational isomer $(70 \%)$

Yields: 40 mg of [III_4_8_M]-linked beads provided $13.0 \mathrm{mg}$ of pure [III_4_8_B]: $81 \%$ 


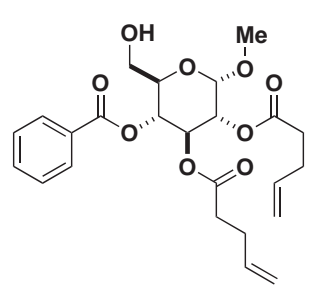

I $21 \mathrm{M}$

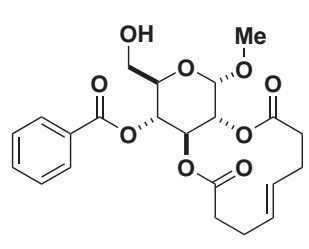

$121 \mathrm{~B}$

\section{[I_2_1_M]}

$\mathbf{t}_{\mathbf{R}}(\mathrm{LC} / \mathrm{MS}): 11 \mathrm{~min}$.

${ }^{1} \mathbf{H}$ NMR(500 MHz, $\left.\mathrm{CDCl}_{3}\right): \delta 2.15-2.30(\mathrm{c}, 4 \mathrm{H}), 2.32-2.50(\mathrm{c}, 4 \mathrm{H}), 3.43(\mathrm{~s}, 3 \mathrm{H}), 3.66(\mathrm{dd}, 1 \mathrm{H}, J=3, J=$ 13), $3.75(\mathrm{~d}, 1 \mathrm{H}, J=13), 3.89(\mathrm{dd}, 1 \mathrm{H}, J=3, J=10), 4.72-5.30(\mathrm{c}, 5 \mathrm{H}), 5.07$ (d, 1H, $J=4.0), 5.23$ (app t, $1 \mathrm{H}, J=10), 5.54-5.84$ (c, $2 \mathrm{H}, J=10), 5.80$ (app t, $1 \mathrm{H}, J=10), 7.45$ (app t, 2H, $J=7.5), 7.59$ (app t, $1 \mathrm{H}, J$ $=7), 7.99(\mathrm{~d}, 1 \mathrm{H}, J=7.5)$

${ }^{13}$ C NMR $\left(101 \mathrm{MHz}, \mathrm{CDCl}_{3}\right): \delta 28.7,28.8,33.4,33.5,55.7,61.2,69.5,69.8,69.8,71.2,97.1,115.7$, $115.9,128.7,128.8,128.8,130.3,130.4,134.1,136.2,136.4,166.6,172.2,172.4$

IR (NaCl): 2935 (w), 1744 (s), 1451 (w), 1269 (m), 1165 (w), 1055 (m), $712(\mathrm{w}) \mathrm{cm}^{-1}$.

RMS-ESI (m/z): [M+H]+ calcd for $\mathrm{C}_{24} 4 \mathrm{H}_{31} \mathrm{O} 9,463.1968$; found, 463.1959 .

\section{[I_2_1_B]}

$\mathbf{t}_{\mathbf{R}}$ (LC/MS): $10.2 \mathrm{~min}$.

${ }^{1}$ H NMR (500 MHz, $\mathrm{CDCl}_{3}$ ): $\delta$ 2.15-2.52 (c, 8H), 2.69 (br s, 1H), 3.44 (s, 3H), 3.66 (br d, 1H, $J=13$ ), $3.75(\mathrm{~d}, 1 \mathrm{H}, J=13), 3.89$ (br d, 1H, $J=10), 4.73$ (d, 1H, $J=4.0), 5.11$ (dd, $1 \mathrm{H}, J=4.0, J=10), 5.21$ (app t , 1H, $J=10), 5.32-5.43(\mathrm{c}, 2 \mathrm{H}), 5.80(\operatorname{app~t}, 1 \mathrm{H}, J=10), 7.45$ (app t, 2H, $J=7.5), 7.59$ (app t, 1H, $J=7), 7.99(\mathrm{~d}, 1 \mathrm{H}, J=7.5)$

${ }^{13}$ C NMR (101 MHz, $\left.\mathrm{CDCl}_{3}\right): \delta$ 29.3, 29.7, 34.6, 35.0, 55.7, 61.2, 69.1, 69.9, 69.9, 70.0, 97.5, 128.9, $128.9(2), 129.9,130.1,130.2(2), 134.1,166.8,172.6,172.7$

IR (NaCl): 2933 (w), 1742 (s), $1450(\mathrm{w}), 1268(\mathrm{~m}), 1166(\mathrm{~m}), 1054(\mathrm{~m}), 713(\mathrm{w}) \mathrm{cm}^{-1}$.

HRMS-ESI $(\mathrm{m} / \mathrm{z}):[\mathrm{M}+\mathrm{H}]^{+}$calcd for $\mathrm{C}_{22} \mathrm{H}_{27} \mathrm{O} 9,435.1655$; found, 435.1657 .

Yields: 40 mg of [I_2_1_M]-linked beads provided $9.8 \mathrm{mg}$ of pure [I_2_1_B]: $88 \%$ 


\section{Assay methods, error modeling and data analysis}

We performed benchmarking and calibration experiments for instruments that collectively provide pin-transfer, liquid-handling, and fluorescence/luminescence detection capabilities. Using a pilot library of 4000 small molecules, including the carbohydrate-derived heterocycles described in this study as well as small molecules with known mechanisms of action $(\sim 10 \%)$, we collected $\sim 200 \mathrm{~K}$ duplicate compound measurements comprising 94 cell-based assays using 4 different assay readouts. These data-collection efforts represent a prototypical dataset for multidimensional screening, ${ }^{15-17}$ and will soon be made available via ChemBank (http://chembank.med.harvard.edu).

\section{High-throughput cell-based assays}

Cell-based assays using either antibody-based cytoblot methods $18-20$ or cell-permeant fluorescent (or fluorogenic) dyes21-24 have been used extensively in HTS of small-molecule collections (see also http://www.probes.com/handbook/). Using such methods with commercially available reagents, we have validated for HTS four cell-based assays ${ }^{15}$ that report on several different cellular processes: 1) a cytoblot against incorporated 5-bromodeoxyuridine $(\mathrm{BrdU}),{ }^{18}$ which reports on DNA synthesis, 2) a cell-viability assay using calcein-(AM) fluorescent dye, ${ }^{21}$ which reports on intracellular esterase activity, 3) a metabolic assay using JC$1,22,25$ a ratiometric sensor of mitochondrial membrane potential, and 4) a redox assay using C12resazurin, 23,26 which senses intracellular reducing equivalents.

All HTS experiments involved cell culture in 384-well microtiter plates. A549 human small-cell lung carcinoma cells (2000/well) were cultured for $24 \mathrm{hr}$ following compound pintransfer in Dulbecco's modified Eagle medium (DMEM) containing 0.5\% fetal bovine serum (FBS), 100 units/mL penicillin, and $1 \mathrm{mg} / \mathrm{mL}$ streptomycin. 125-nL aliquots of compound were transferred from 6-mM arrayed stock solutions using a TekCel Automated TekBench (TekCel, Inc.; Hopkinton, MA) equipped with a 384-member non-slotted, floating pin array (V \& $\mathrm{P}$ Scientific; San Diego CA). Cell cultures were maintained in a humidified incubator at 37C and $5 \% \mathrm{CO}_{2}$. BrdU cytoblots were performed as described by Stockwell,18 modified by Wagner. ${ }^{27}$ For experiments with fluorogenic dyes, a dilute solution of dye in phosphate-buffered saline (PBS) was added to cultures and incubated for 30-120 min either at room temperature or 37C, after which this solution was removed and the plate washed twice with PBS. Luminescence or fluorescence intensity readings were made with an Analyst-HT (Molecular Devices; Sunnyvale, CA) plate reader with appropriate dichroic mirrors and excitation/emission filter combinations. 


\section{Specific information for calcein-(AM) assay}

Calcein-(AM) is a fluorogenic, membrane-permeant probe that is converted to an anionic fluorescent form upon cleavage by intracellular esterases, and is thus retained by live cells. Measured fluorescence signal (ex. 485/em. 530) is essentially proportional to the number of live cells (data not shown), with the caveat that low signal could also indicate a permeablized membrane, direct inhibition of esterases, or be confounded by autofluorescence of the sample. Following pin-transfer and incubation with compounds, a dilute solution of calcein-(AM) in PBS was added to cells at a final concentration of $1 \mu \mathrm{M}$. Plates were incubated at room temperature for $45 \mathrm{~min}$, and washed with PBS before fluorescence detection.

\section{Specific information for C12-resazurin assay}

C12-resazurin is a fluorogenic, membrane-permeant probe that is converted to a fluorescent form upon reduction by intracellular reducing agents. C12-resazurin is converted to the fluorescent dye resorufin upon reduction within the cell; the resultant fluorescence (ex. 530/em. 585) is proportional to the level of intracellular reducing agents (primarily NADH). Following pin-transfer and incubation with compounds, a dilute solution of C12-resazurin in PBS was then added to cells at a final concentration of $2 \mu \mathrm{M}$. Plates were incubated at room temperature for $30 \mathrm{~min}$, and washed with PBS before fluorescence detection.

\section{Specific information for $\mathrm{JC}-1$ assay}

JC-1 is a membrane-permeant probe that binds to mitochondrial membranes within cells and fluoresces green as an individual molecule (ex. 485/em. 530), but is converted to a red fluorescent form (ex. 530/em. 585) when it is internalized in a voltage-dependent manner across the mitochondrial inner membrane, forming so-called "J-aggregates".28 The ratio of red to green signal is thus an indicator of mitochondrial membrane potential, and it was these ratios that we used as raw data to initiate error-modeling (see below). Increases in this ratio indicate a loss of potential and poorly functioning mitochondria. ${ }^{29}$ Following pin-transfer and incubation with compounds, a dilute solution of JC-1 in PBS was then added to cells at a final concentration of $1 \mu \mathrm{M}$. Plates were incubated at 37C for $2 \mathrm{hr}$, and washed with PBS before fluorescence detection. 


\section{Chemical-genetic modifier screens}

For chemical-genetic modifier screens, ${ }^{16}$ biological modulators of known activity were added to cells at the time of seeding in microtiter plates, immediately prior to library compound addition by pin-transfer. Concentrations of known biological modulators used were:

\begin{tabular}{|c|c|c|}
\hline 1 & $1-\beta$-D-arabinofuransylcytosine & $2 \mu \mathrm{M}$ \\
\hline 2 & brefeldin A & $2 \mu \mathrm{M}$ \\
\hline 3 & butein & $10 \mu \mathrm{M}$ \\
\hline 4 & castanospermine & $2 \mu \mathrm{M}$ \\
\hline 5 & carbonyl cyanide $m$-chlorophenylhydrazone & $2 \mu \mathrm{M}$ \\
\hline 6 & chloroquin & $2 \mu \mathrm{M}$ \\
\hline 7 & colcemid & $2 \mu \mathrm{M}$ \\
\hline 8 & cycloheximide & $2 \mu \mathrm{M}$ \\
\hline 9 & cytochalasin & $1 \mu \mathrm{M}$ \\
\hline 10 & 4,5-dianilinophthalamide & $10 \mu \mathrm{M}$ \\
\hline 11 & emetine & $2 \mu \mathrm{M}$ \\
\hline 12 & forskolin & $2 \mu \mathrm{M}$ \\
\hline 13 & genistein & $56 \mu \mathrm{M}$ \\
\hline 14 & hydroxy-2-naphthalenylmethylphosphonic a & acetoxymethyl ester \\
\hline & & $5 \mu \mathrm{M}$ \\
\hline 15 & hydroxyurea & $2 \mu \mathrm{M}$ \\
\hline 16 & lysophosphatidic acid & $2 \mu \mathrm{M}$ \\
\hline 17 & valinomycin & $2 \mu \mathrm{M}$ \\
\hline
\end{tabular}




\section{Analysis of HTS data}

We have developed standard data analysis methods for multidimensional screening 15 based on HTS assays using photon-counting readouts (e.g., fluorescence, luminescence, and absorbance). We applied these standards to all experiments in this study. Briefly, a substantial number of baseline "mock-treatment" controls were performed with each assay, deriving from DMSO controls both co-arrayed on the same plates with library compounds and independently arrayed. Measurements are made within the linear range of instrument detection to promote the validity of an assumption of distribution symmetry. This is especially important for modifier screens, in which detection of both low- and high-signal outliers is desired. To account for plate-to-plate variation in signal, ${ }^{30}$ median raw values of control well signals are first subtracted from all raw values (i.e., control measurements and compound measurements) on the same plate, providing a common distribution centroid (approaching zero) for all plates in one experiment, which we define as a collection of distinct probe source plates (e.g., a multimicrotiter plate library ${ }^{31}$ ) exposed to the same assay conditions. Next, the (now-relative) values for all control measurements in the experiment are collected together, and values failing Chauvenet's criterion ${ }^{32}$ for this overall distribution are discarded to protect against the overcontribution of edge effects, known to present a technical problem for microtiter plate experiments. ${ }^{33,34}$ The standard deviation of the distribution derived from the remaining control measurements is used as a per-experiment normalization value $\left(\sigma_{\text {assay }}\right)$ to evaluate independently each background-subtracted value for a compound measurement in that experiment. By dividing background-subtracted compound measurements by $\sigma_{\text {assay, }}$ each compound is placed along a dimensionless probabilistic measurement axis that directly corresponds to the likelihood $\left(P_{\text {noise }}\right)$ that the measurement can be explained by assay noise. In this study, Z-scores along this axis, corresponding to the (signed) number of standard deviations each compound fell from the baseline distribution, were used as the continuousvalued $Z$-score. By establishing a threshold value (e.g., $\left.P_{\text {noise }}<0.01\right)$ for binning measurement outcomes, we generated outcome bins corresponding to "active" (i.e., low-signal or high-signal outliers) and "inactive" (i.e., indistinguishable from noise) compounds. By binning, we were able to assign the activities presented in Figure $5 a$. Using the continuous-valued Z-scores, we are able to construct the multidimensional chemical-genetic fingerprints presented in Figure $5 c$ e. 


\section{Statistical Analysis}

The compounds are grouped into pairs indexed by an integer $j$. Each pair consists of 2 compounds in classes $\{B, M\}$, corresponding to the bicyclic and monocyclic classes.

For each class $C \in\{B, M\}$ we obtained counts $n_{j}^{C}$ of the number of assays in which each compound scored as positive. Defining

$$
n_{j}^{T}=n_{j}^{B}+n_{j}^{M}
$$

as the total number of successful assays for the pair $j$, we fit a compound Gamma-Poisson model. $n_{j}^{C}$ is taken to be Poisson distributed with (hidden) mean $\mu_{j}^{C}$.

$\mu_{j}^{C}$ has a prior Gamma distribution with parameters $p_{C}, \lambda_{C}$ which are independent of $j$.

Thus

$$
P\left(\mu=\mu_{j}^{C}\right)=\frac{\mu^{p_{C}-1} e^{-\lambda_{C}}\left(\lambda_{C}\right)^{p_{C}}}{\Gamma\left(p_{C}\right)}
$$

and

$$
P\left(n=n_{j}^{C}\right)=\int_{0}^{\infty} \frac{\mu^{n+p_{C}-1} e^{-\left(\lambda_{C}+\mu\right)}\left(\lambda_{C}\right)^{p_{C}}}{\Gamma\left(p_{C}\right) n !} d \mu
$$

For each class $C \in\{T, B, M\}$ we can now estimate $p_{C}, \lambda_{C}$ by maximum likelihood (there is an EM iteration $^{35}$ whose details are omitted). Let $L(C)$ be the log-likelihood of the data with the maximum likelihood parameters. Then we can form:

$$
S=2(L(B)+L(M)-L(T))
$$

$S$ is a likelihood ratio statistic, and standard theory would suggest that under the null in which the classes $B$ and $M$ are indistinguishable in the assays, $S$ would be chi-square distributed with 2 degrees of freedom. There are however serious modeling issues, especially the likely non-independence of the assays, and we preferred to compute a $P$-value using permutation testing as described in the text and in the legend to Figure 5. 


\section{References}

1. Pangborn, A. B.; Giardello, M. A.; Grubbs, R.H.; Rosen, R. K.; Timmers, F. J. Organometallics, 1996, $15,1518-1520$.

2. Tallarico, J. A.; Depew, K. D.; Pelsih, H. E.; Westwood, N, J.; Lindsley, C. W.; Shair, M. D.; Schreiber, S. L.; Foley, M. A. J. Comb. Chem. 2001, 3, 312-318.

3. (a) Hoye, T. R.; Hanson, P. R.; Vyvyan, J. R. J. Org. Chem. 1994, 59, 4096-4103. (b) Gottlieb, H. E.; Kotlyar, V.; Nudelman, A. J. Org. Chem. 1997, 62, 7512-7515.

4. Ferro, V.; Mocerino, M.; Stick, R. V.; Tilbrook, D. M. G. Aust. J. Chem. 1988, 41, 813-815.

5. Ogawa, T.; Matsui, M. Tetrahedron. 1981, 37, 2363-2369.

6. Hense, A.; Ley, S. V.; Osborn, H. M. I.; Owen, D. R.; Poisson, J. R.; Warriner, S. L.; Wesson, D. E. J. Chem. Soc., Perkin Trans. 1. 1997, 2023-2030.

7. Rashid, A.; Mackie, W.; Colquhoun, I. J.; Lamba, D. Can. J. Chem. 1990, 68, 1122-1127.

8. (a) Oriyama, T.; Ohashi, K.; Oriyama, T. Synthesis. 1999, 7, 1141-1144. (b) Hu, G.; Vasella, A. Helv. Chim. Acta. 2003, 86, 4369-4391.

9. Boger, D. L.; Honda, T. J. Am. Chem. Soc. 1994, 116, 5647-5656.

10. Wong, C-H.; Ye, X-S.; Zhang, Z. J. Am. Chem. Soc. 1998, 120, 7137-7138.

11. Myers, A. G.; Yang, B. H.; Chen, H.; McKinstry, L.; Kopecky, D. J.; Gleason, J. L. J. Am. Chem. Soc. 1997, 119, 6496-6511.

12. Evans, D. A.; Bartroli, J. Tetrahedron. Lett. 1982, 23, 807-810.

13. Jiao, P.; Xu, J.; Zhang, Q., Choi, M. C. K.; Chan, A. S. C. Tetrahedron. Asymmetry. 2001, 12, 3081-3088.

14. Grubbs, RH et al, Tetrahedron. Lett. 1999, 40, 4137-4139

15. A detailed description of multidimensional screening (including its conceptual basis, detailed assay development and error-modeling methods, and its role in hypothesis-generation) will be 
presented elsewhere: Lamenzo, J. O.; Dean, E. F.; Gorenstein, J.; Kim, Y.-K.; Wagner, B. K.; Schreiber, S. L.; Clemons, P. A., manuscript in preparation.

16. Koeller, K. M. et al. Chemical genetic modifier screens: small molecule trichostatin suppressors as probes of intracellular histone and tubulin acetylation. Chem. Biol. 2003, 10, 397-410.

17. Haggarty, S. J., Clemons, P. A. and Schreiber, S. L. Chemical genomic profiling of biological networks using graph theory and combinations of small molecule perturbations. J. Am. Chem. Soc. 125, 10543-10545.

18. Stockwell, B. R., Haggarty, S. J. and Schreiber, S. L. High-throughput screening of small molecules in miniaturized mammalian cell-based assays involving post-translational modifications. Chem. Biol. 1999, 6, 71-83.

19. Mayer, T. U. et al. Small molecule inhibitor of mitotic spindle bipolarity identified in a phenotype-based screen. Science 1999, 286, 971-974.

20. Haggarty, S. J. et al. Dissecting cellular processes using small molecules: identification of colchicine-like, taxol-like and other small molecules that perturb mitosis. Chem. Biol. 2000, 7, 275-286.

21. Decherchi, P., Cochard, P. and Gauthier, P. Dual staining assessment of Schwann cell viability within whole peripheral nerves using calcein-AM and ethidium homodimer. J. Neurosci. Methods 1997, 71, 205-13.

22. Andrews, P. A. and Albright, K. D. Mitochondrial defects in cis-diamminedichloroplatinum(II)resistant human ovarian carcinoma cells. Cancer. Res. 1992, 52, 1895-1901.

23. Zalata, A., Hafez, T., Mahmoud, A. and Comhaire, F. Relationship between resazurin reduction test, reactive oxygen species generation, and gamma-glutamyltransferase. Hum. Reprod. 1995, $10,1136-40$.

24. Barnes, S., Spenney, J. G. Stoichiometry of the NADH-oxidoreductase reaction for dehydrogenase determinations. Clin. Chim. Acta. 1980, 107, 149-154.

25. Reers, M. et al. Mitochondrial membrane potential monitored by JC-1 dye. Methods. Enzymol. 1995, 260, 406-417. 
26. Gabrielson, J. et al. Evaluation of redox indicators and the use of digital scanners and spectrophotometer for quantification of microbial growth in microplates. J. Microbiol. Methods. , 2002, 50, 63-73.

27. Wagner, B. K. in Molecular \& Cellular Biology 167 (Harvard University, Cambridge, 2003).

28. Kulkarni, G. V., Lee, W., Seth, A. and McCulloch, C. A. Role of mitochondrial membrane potential in concanavalin A-induced apoptosis in human fibroblasts. Exp. Cell. Res. 1998, 245, 170-178.

29. Troyan, M. B., Gilman, V. R., Gay, C. V. Mitochondrial membrane potential changes in osteoblasts treated with parathyroid hormone and estradiol. Exp. Cell. Res. 1997, 233, 274-280.

30. Brideau, C., Gunter, B., Pikounis, B., Liaw, A. Improved statistical methods for hit selection in high-throughput screening. J. Biomol. Screen. 2003, 8, 634-647.

31. Clemons, P. A. et al. A one-bead, one-stock solution approach to chemical genetics, part two. Chem. Biol. 2001, 8, 1183-1195.

32. Bevington, P. R. and Robinson, D. K. Data Reduction and Error Analysis for the Physical Sciences (McGraw-Hill, Boston, MA, 1991).

33. Gunter, B., Brideau, C., Pikounis, B., Liaw, A. Statistical and graphical methods for quality control determination of high-throughput screening data. J. Biomol. Screen. 2003, 8, 624-633.

34. Lundholt, B. K., Scudder, K. M., Pagliaro, L. A simple technique for reducing edge effect in cellbased assays. J. Biomol. Screen. 2003, 8, 566-570.

35. Dempster, A.P., Laird N.M., Rubin, D.B. J. the Royal Statistical Society B, 1977, 39, 1-38 


\section{NMR spectra}

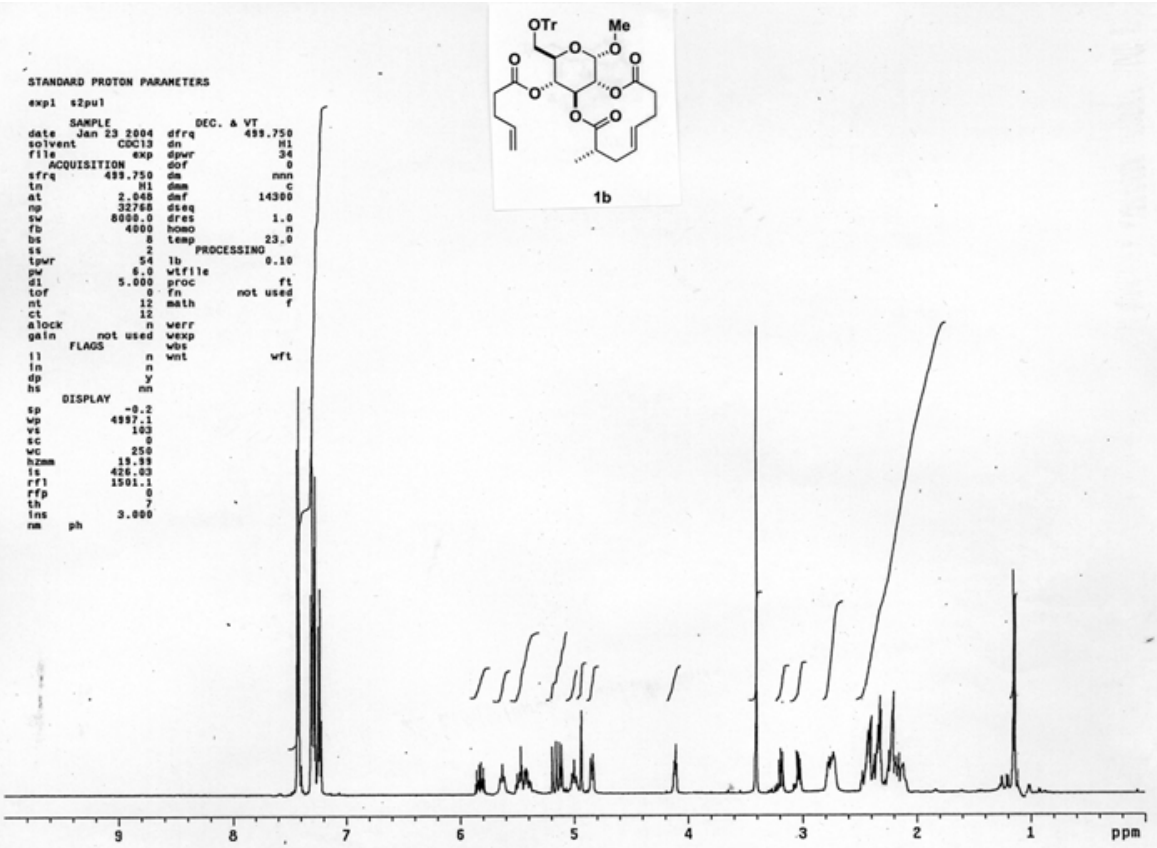

${ }^{1} \mathrm{H}$ NMR spectrum of $1 \mathrm{~b}(500 \mathrm{MHz})$

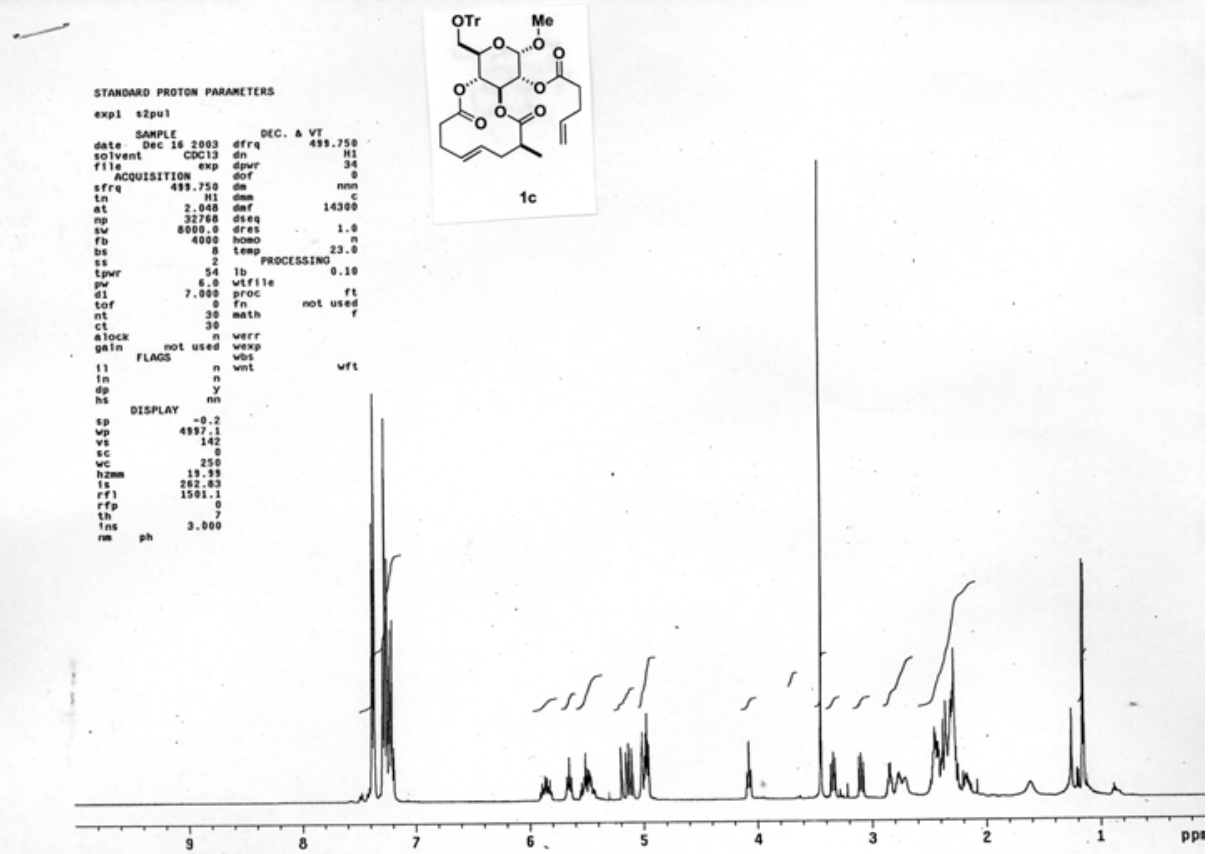

${ }^{1} \mathrm{H}$ NMR spectrum of $1 \mathrm{c}(500 \mathrm{MHz})$ 


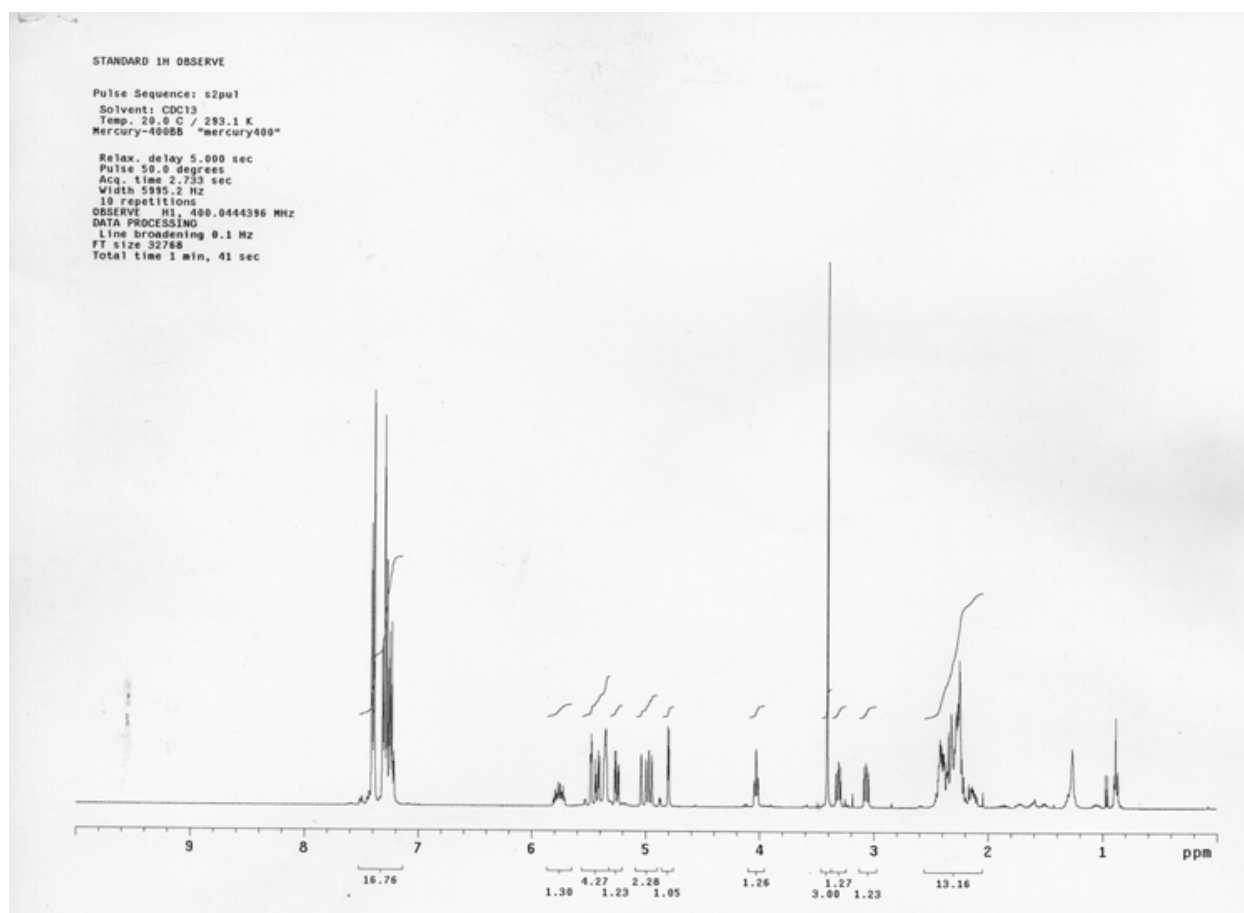

${ }^{1} \mathrm{H}$ NMR spectrum of $2 \mathrm{~b}(500 \mathrm{MHz})$

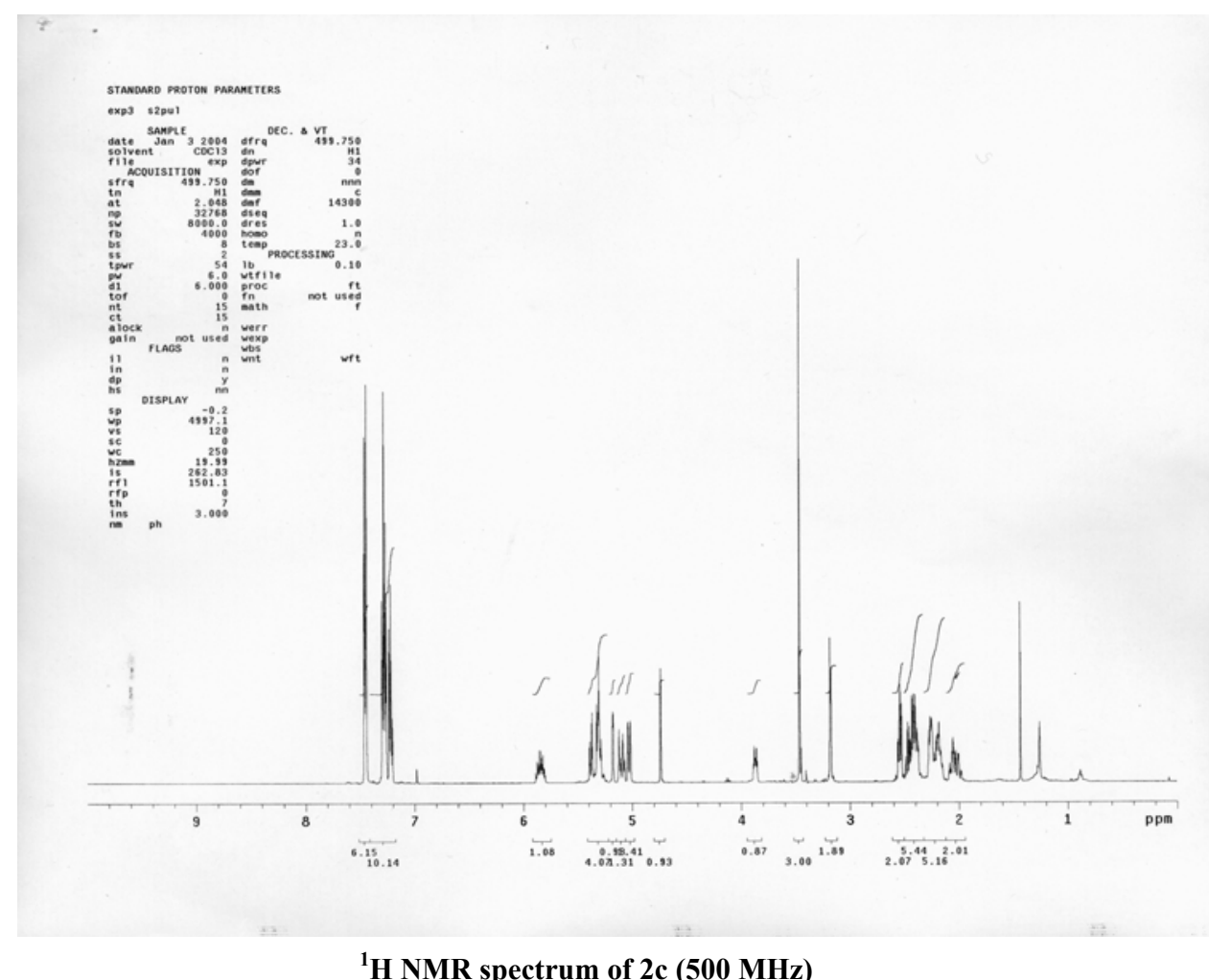

${ }^{1} \mathrm{H}$ NMR spectrum of $2 \mathrm{c}(500 \mathrm{MHz})$ 
$13 \mathrm{C}$ osserve

Pulse Sequencel s2por

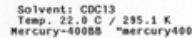

Pulse 25,0 atergets.

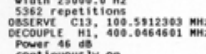

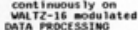

th'ne broagenting $1.0 \mathrm{kz}$

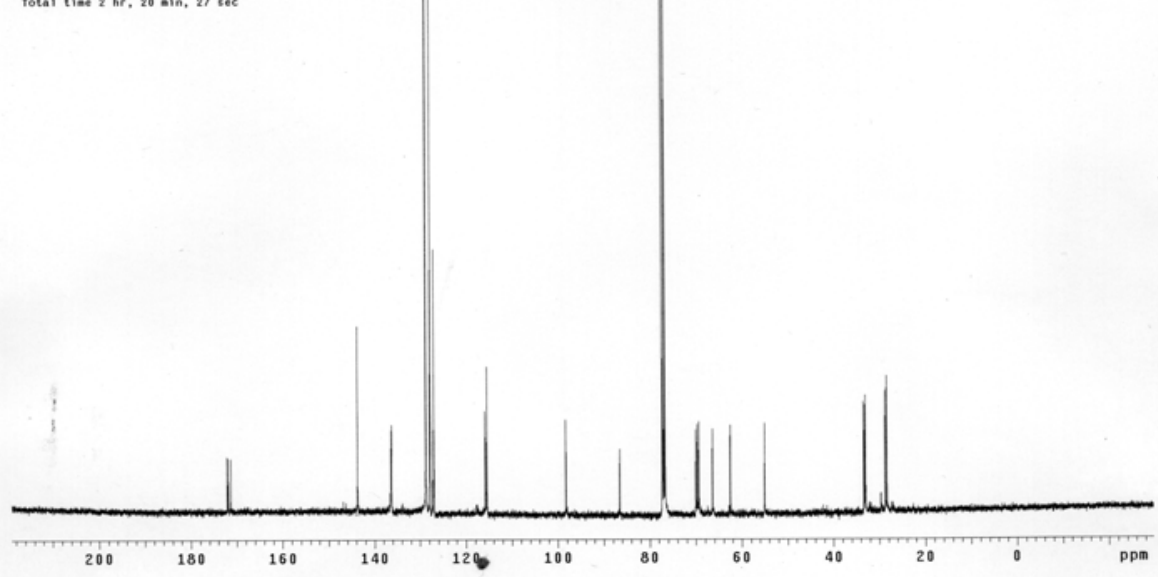

\section{${ }^{13} \mathrm{C}$ NMR spectrum of $2 \mathrm{~b}$}

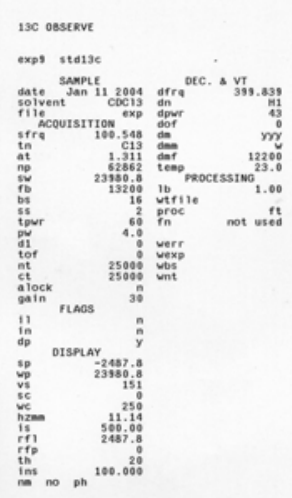

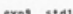
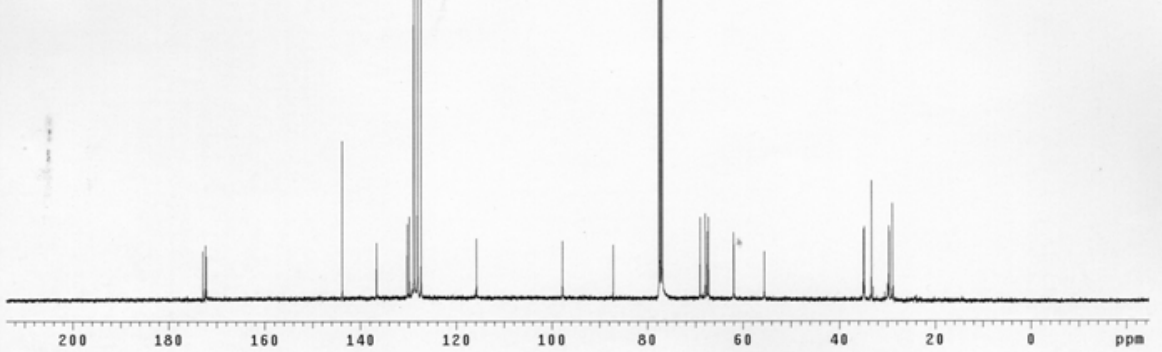

${ }^{13} \mathrm{C}$ NMR spectrum of $2 \mathrm{c}$ 


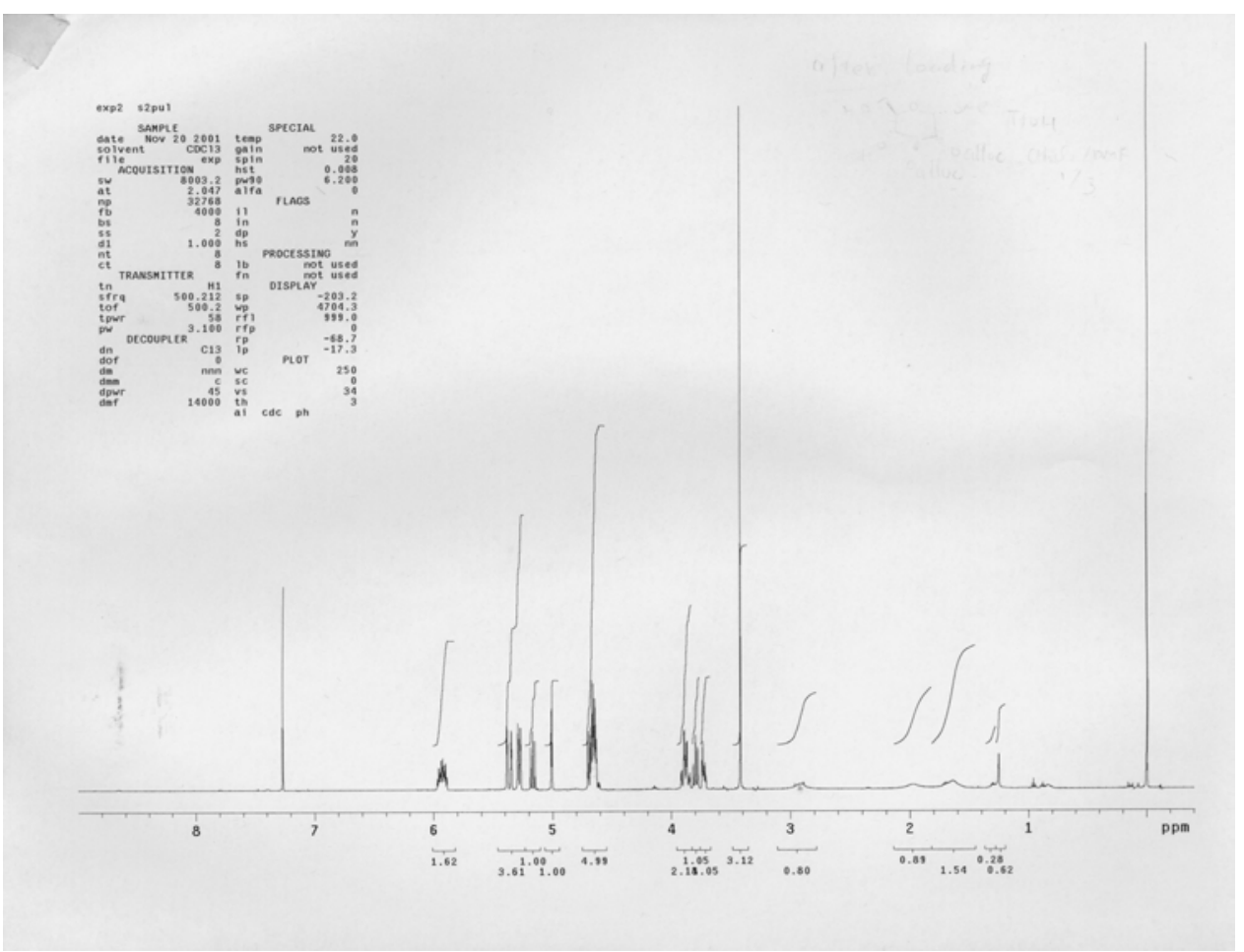

${ }^{1}$ H NMR spectrum of I

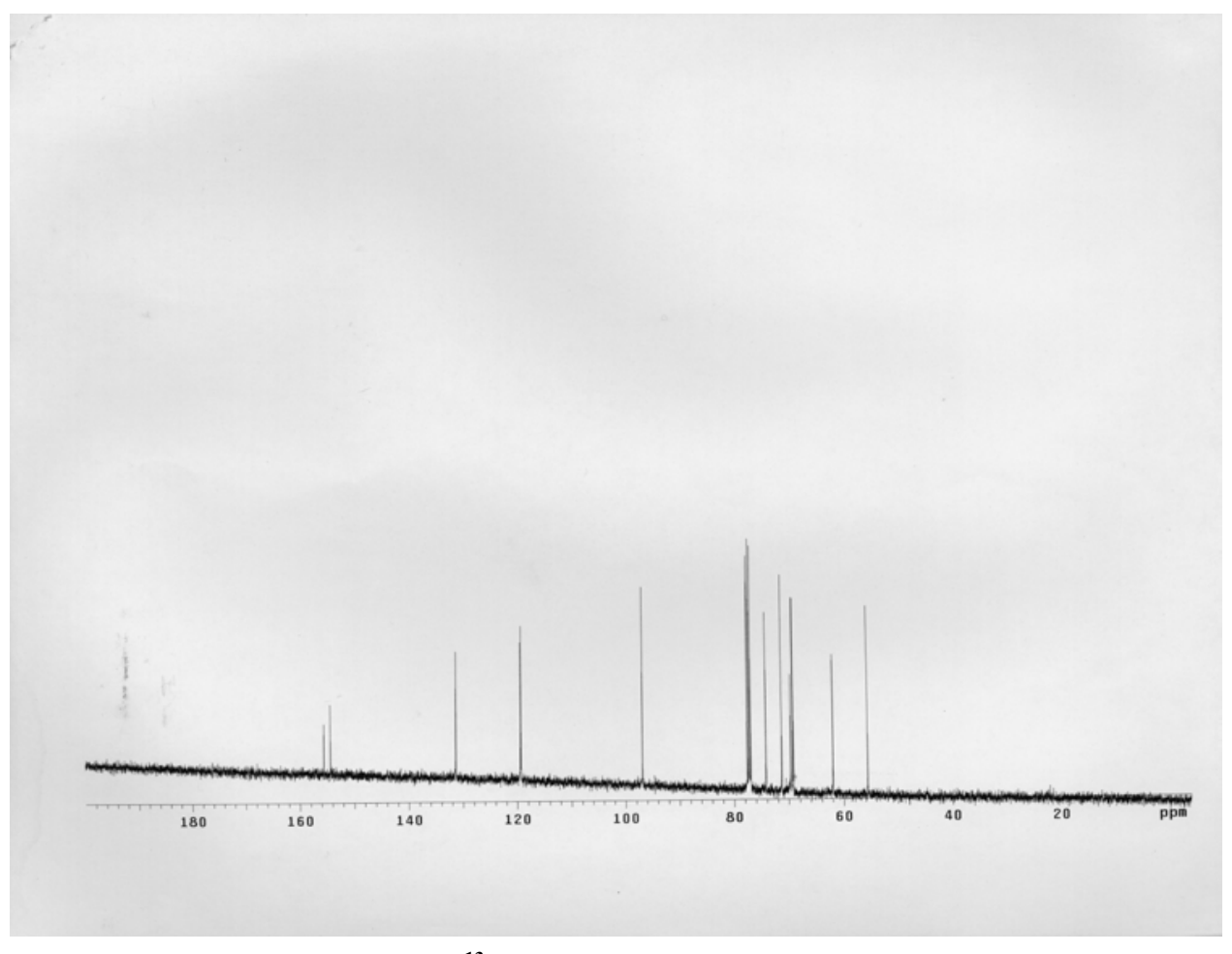

${ }^{13} \mathrm{C}$ NMR spectrum of I 


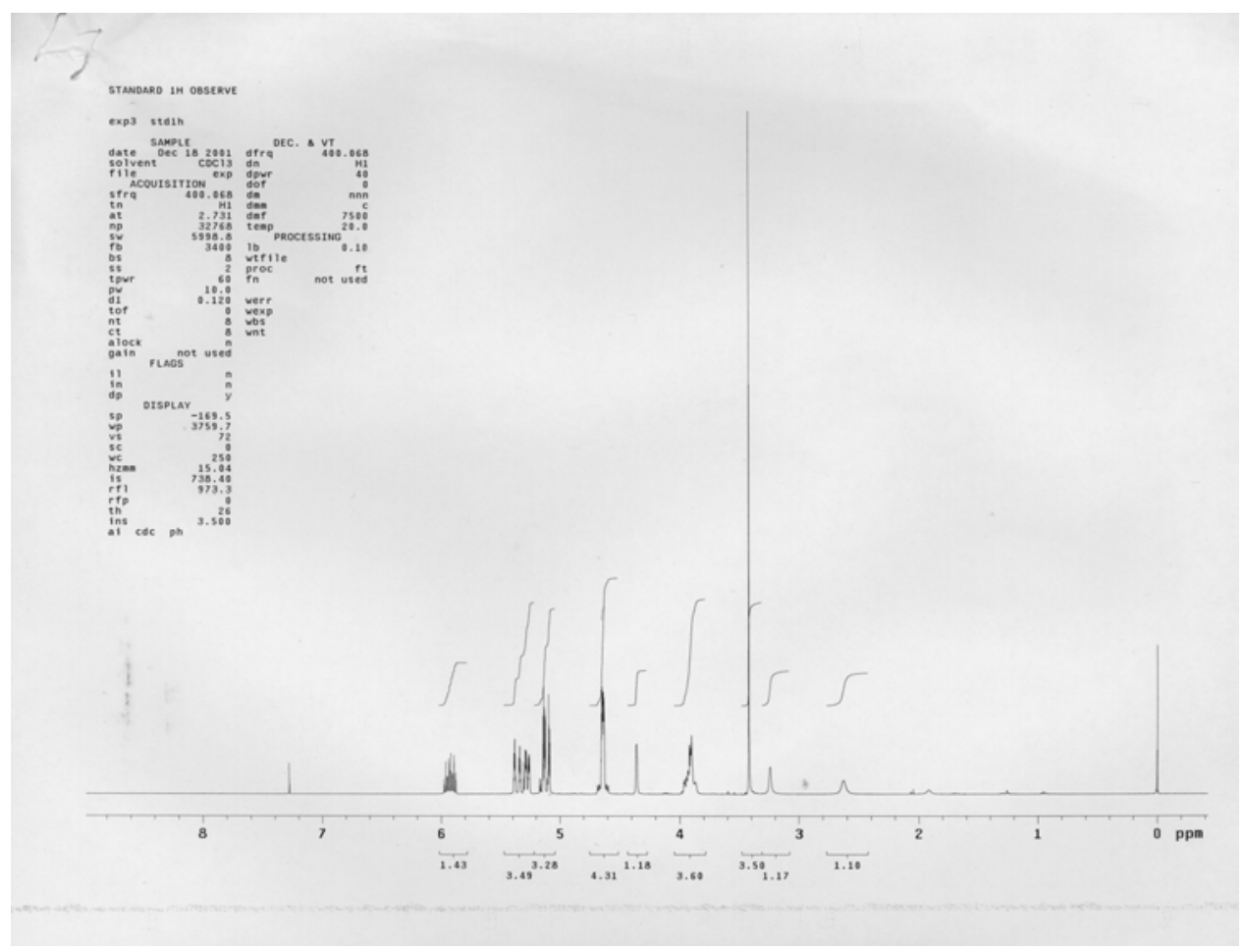

${ }^{1}$ H NMR spectrum of II

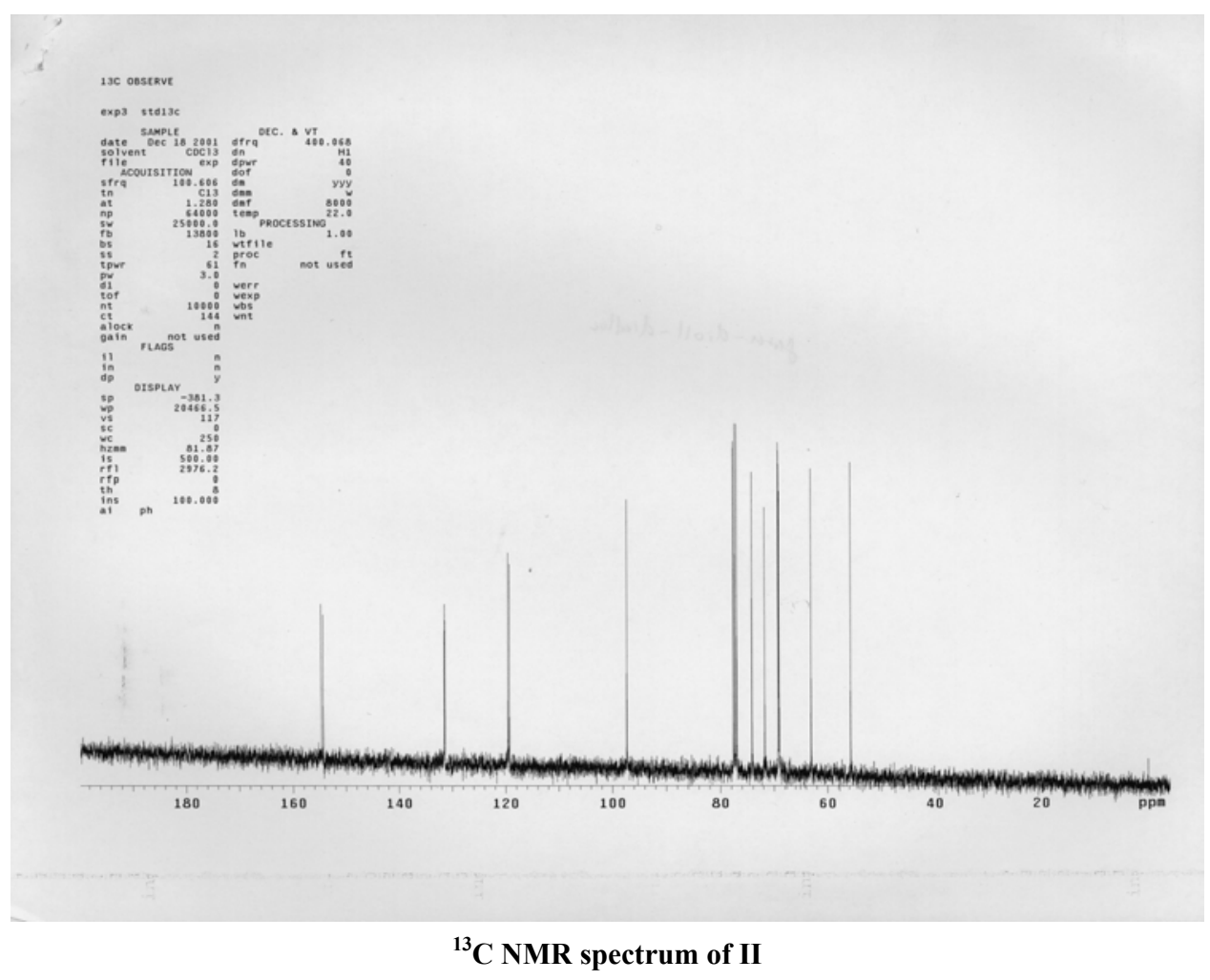




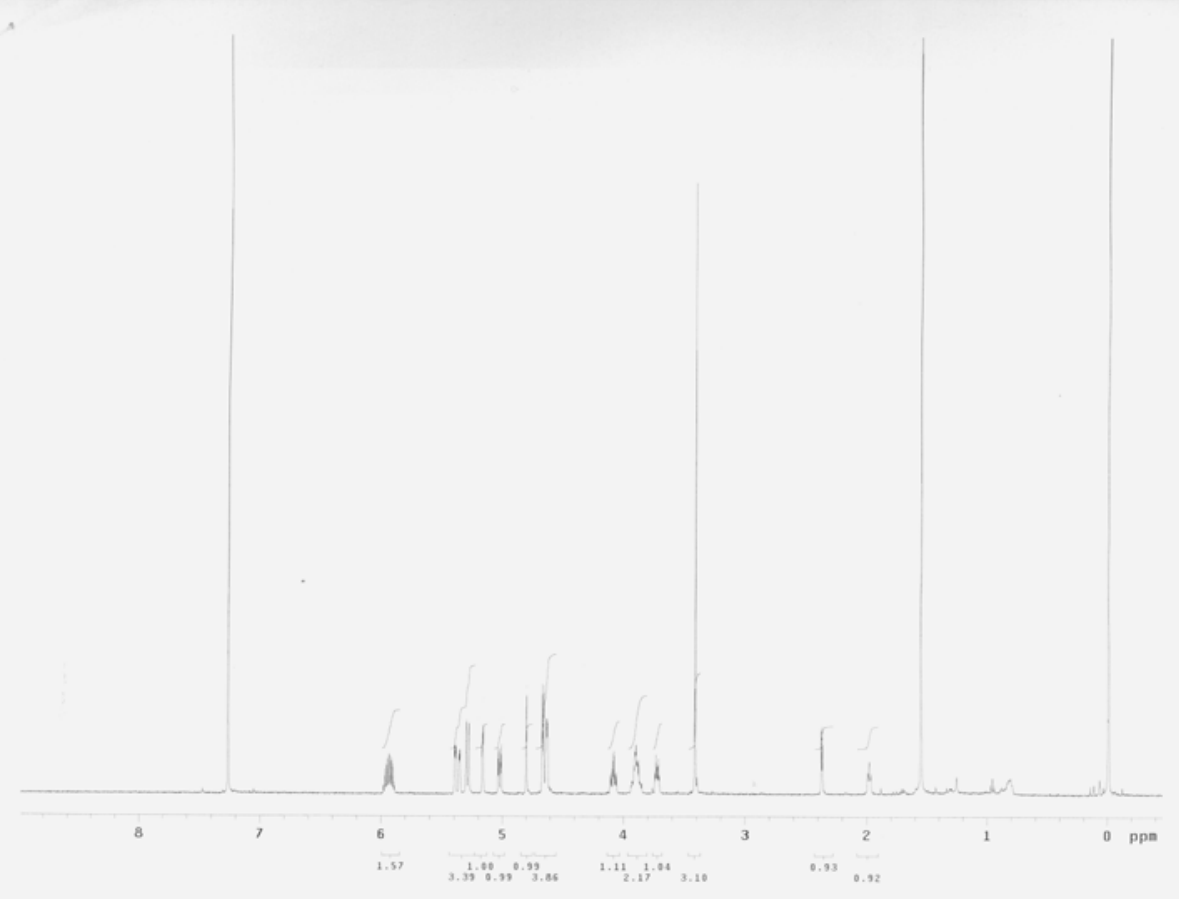

${ }^{1} \mathrm{H}$ NMR spectrum of III

Pulse sequence: s2001

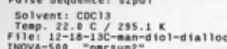

Pulse 36.0 degrest

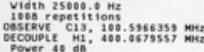

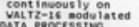

Thing

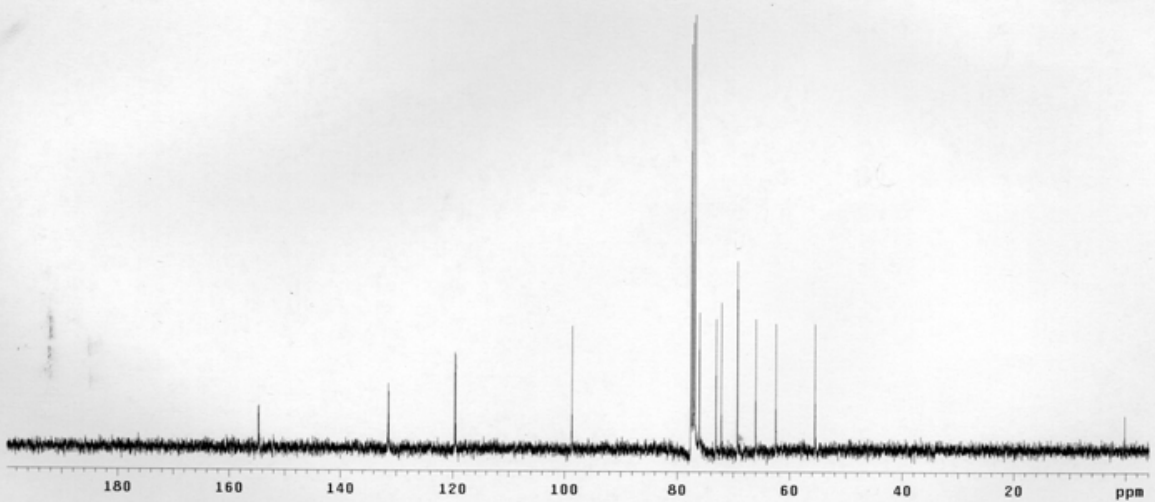

${ }^{13} \mathrm{C}$ NMR spectrum of III 


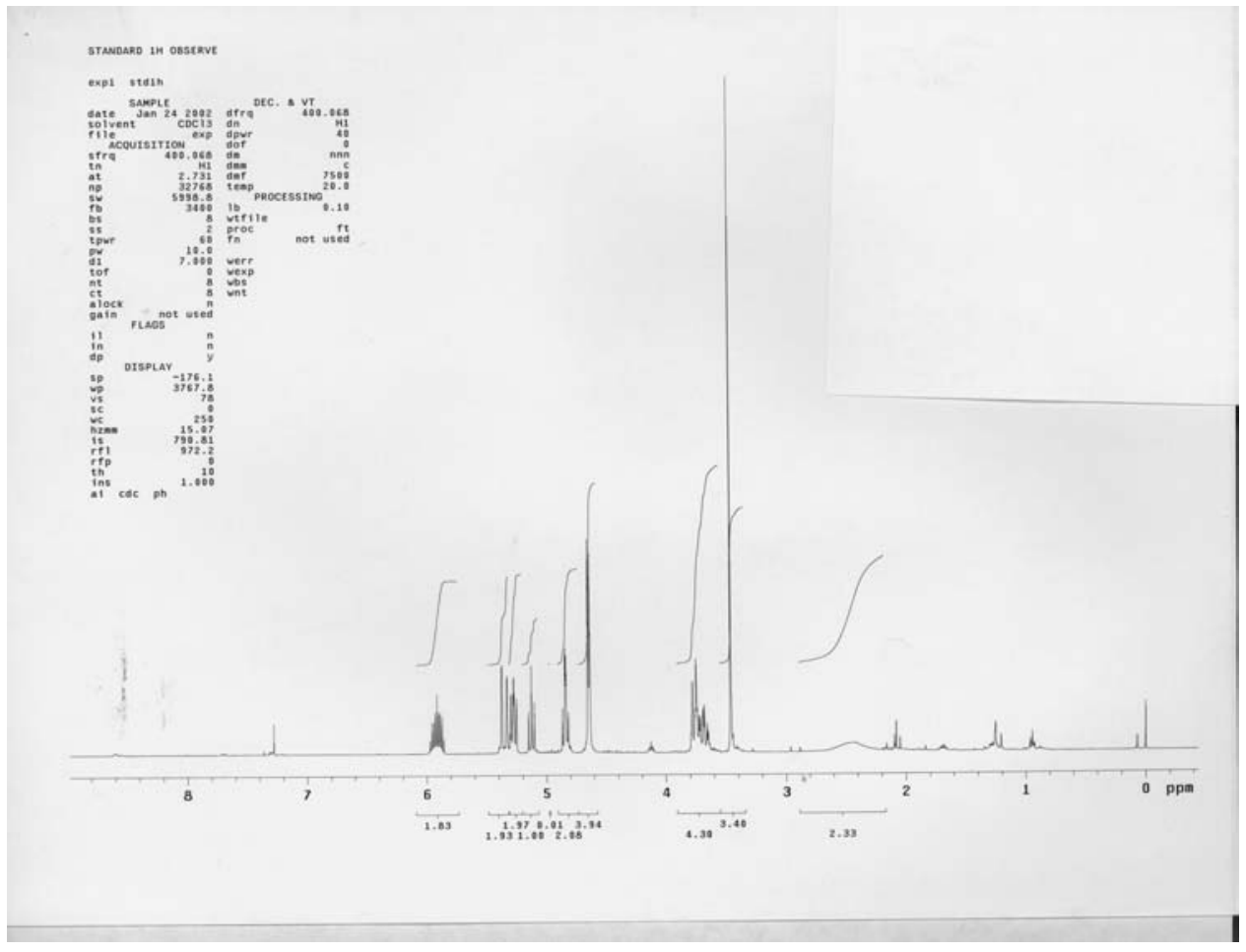

${ }^{1}$ H NMR spectrum of IV

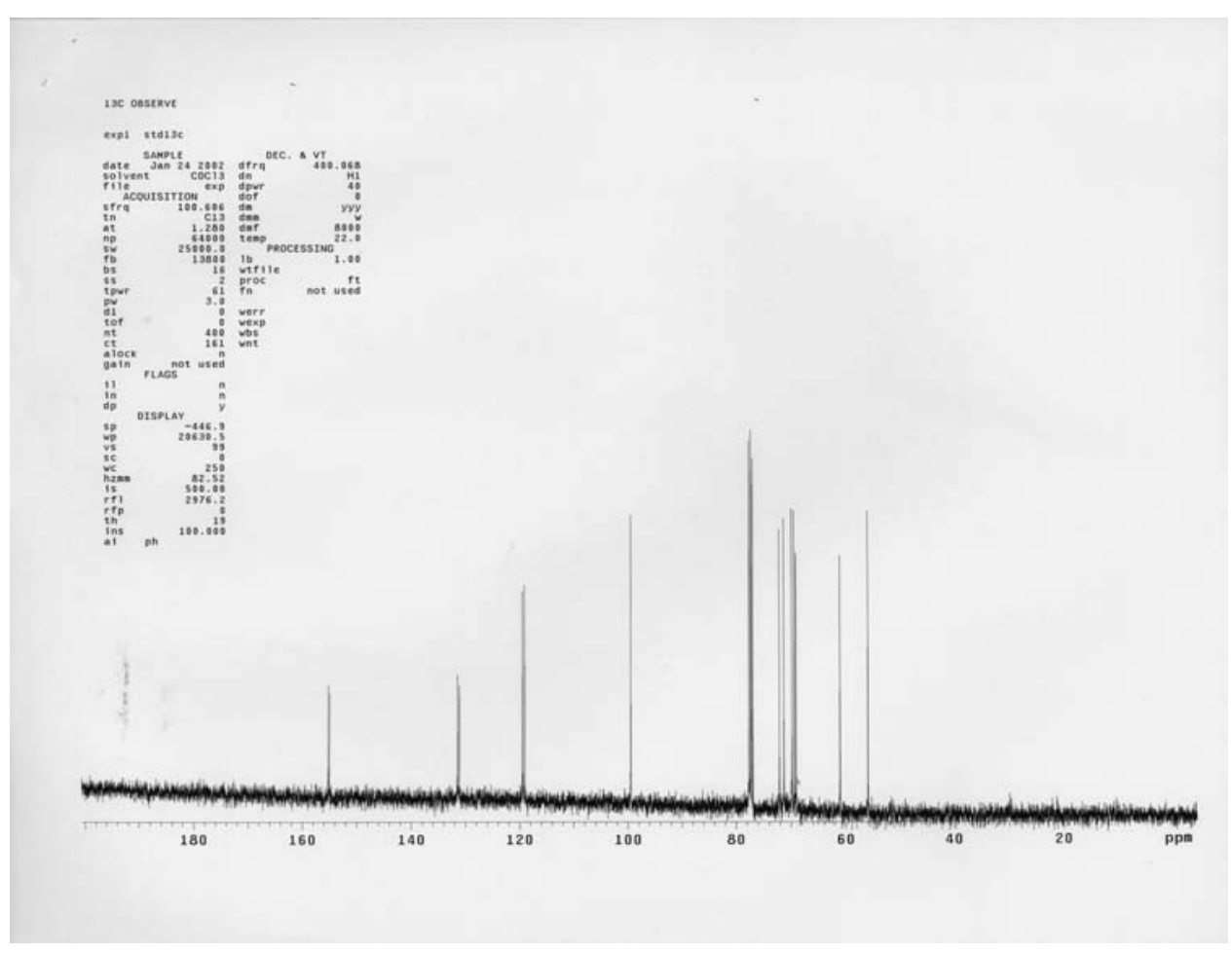

${ }^{13} \mathrm{C}$ NMR spectrum of IV 


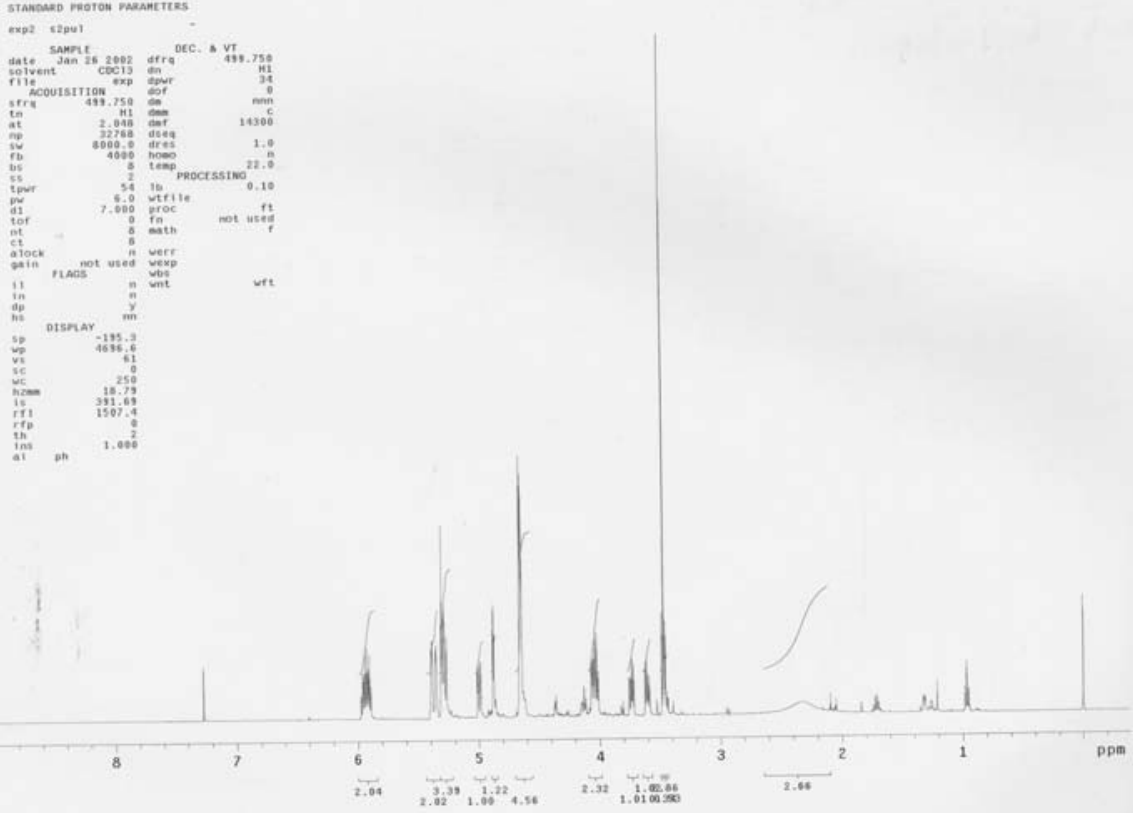

${ }^{1} \mathrm{H}$ NMR spectrum of $\mathrm{V}$

190 ossceve

$\operatorname{expl}$ staise

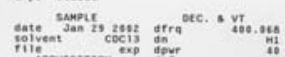

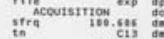

it

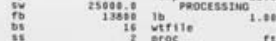

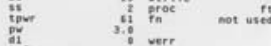

tor

atin naosot ured

î

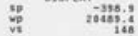

nem

it

ai 1000.000

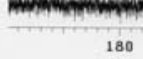

\section{${ }^{13} \mathrm{C}$ NMR spectrum of $\mathrm{V}$}




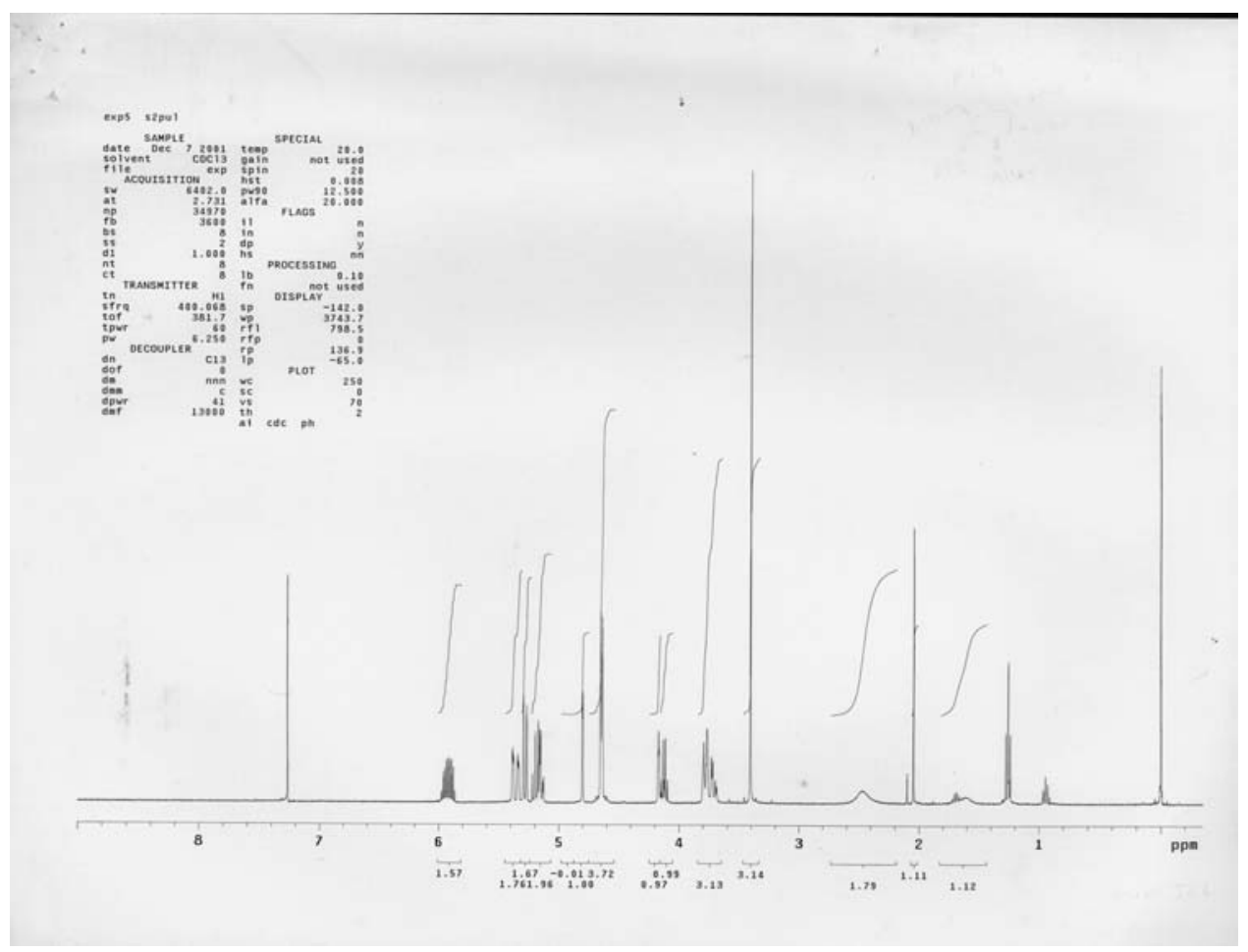

\section{${ }^{1}$ H NMR spectrum of VI}

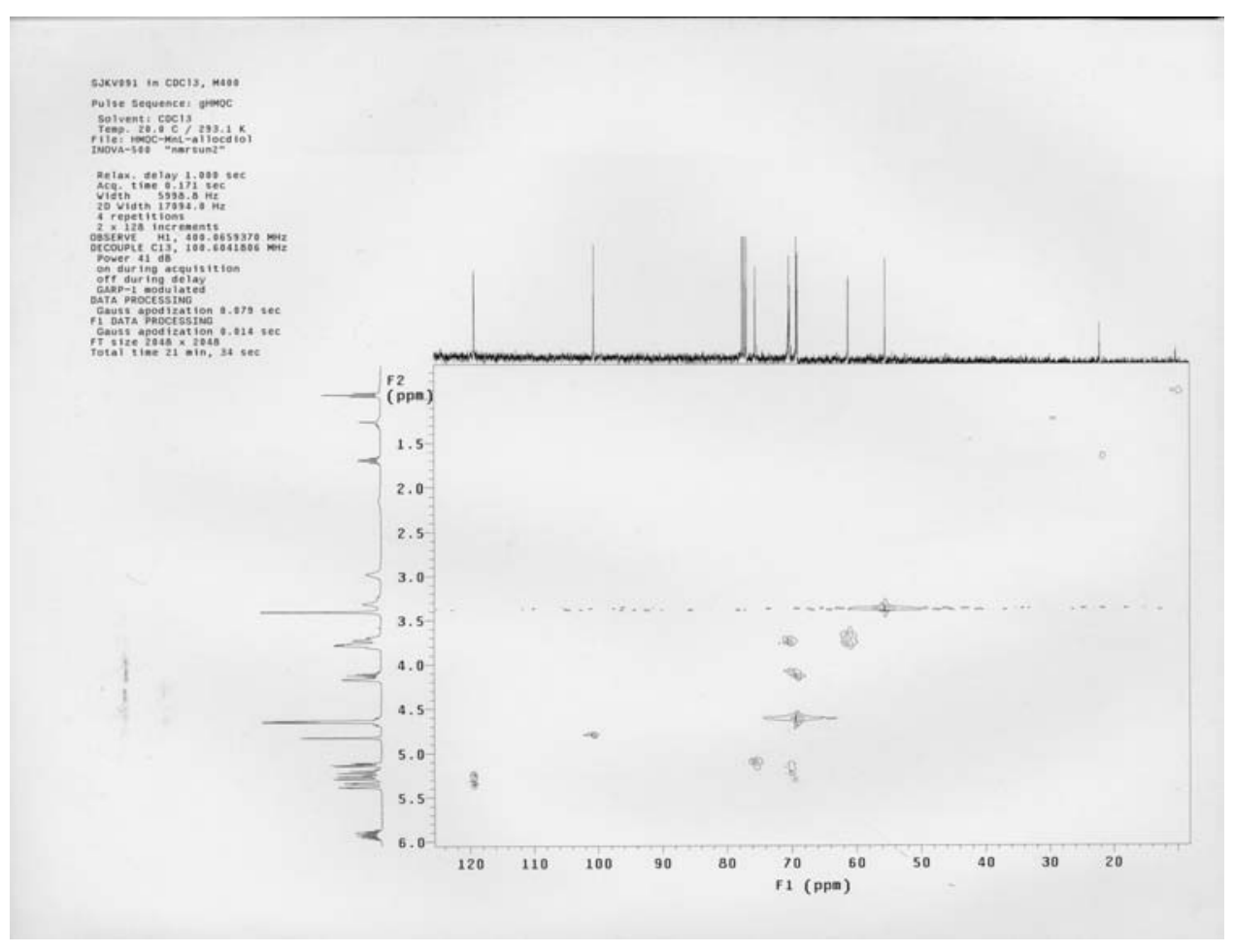

gHMQC spectrum of VI 


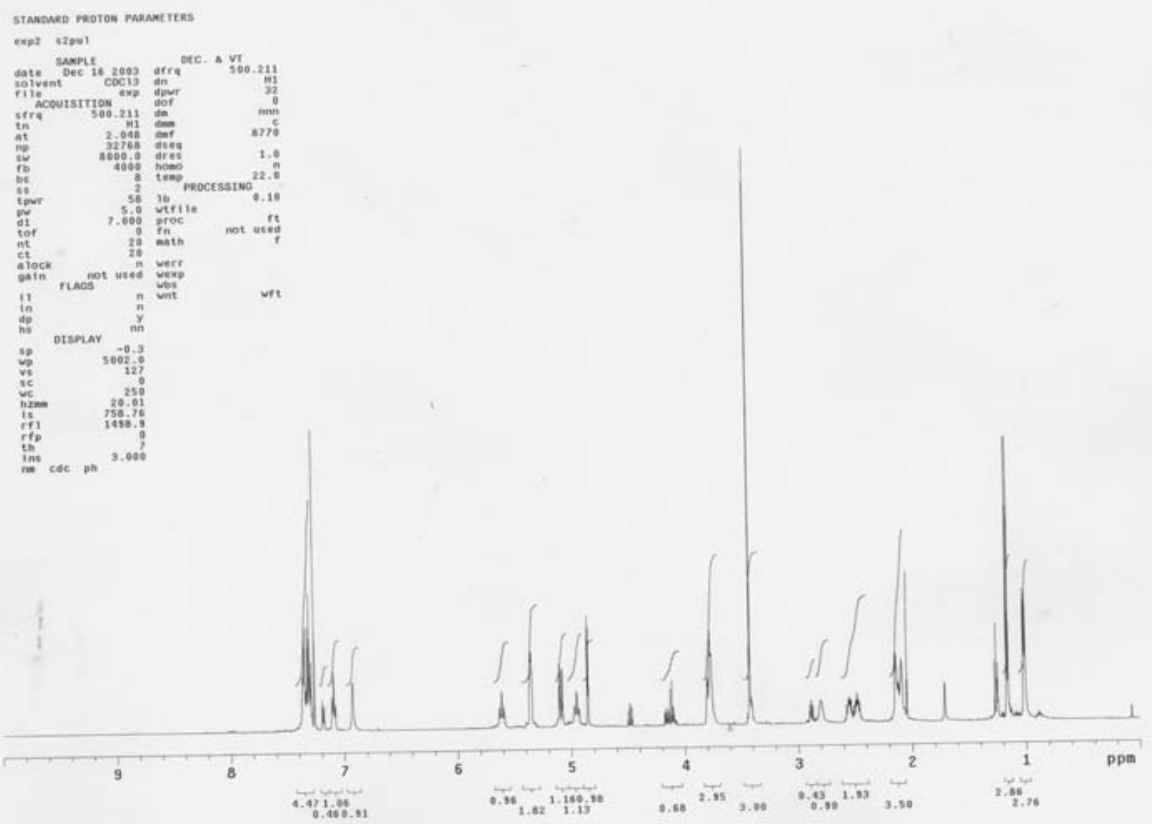

${ }^{1}$ H NMR spectrum of I 12 B

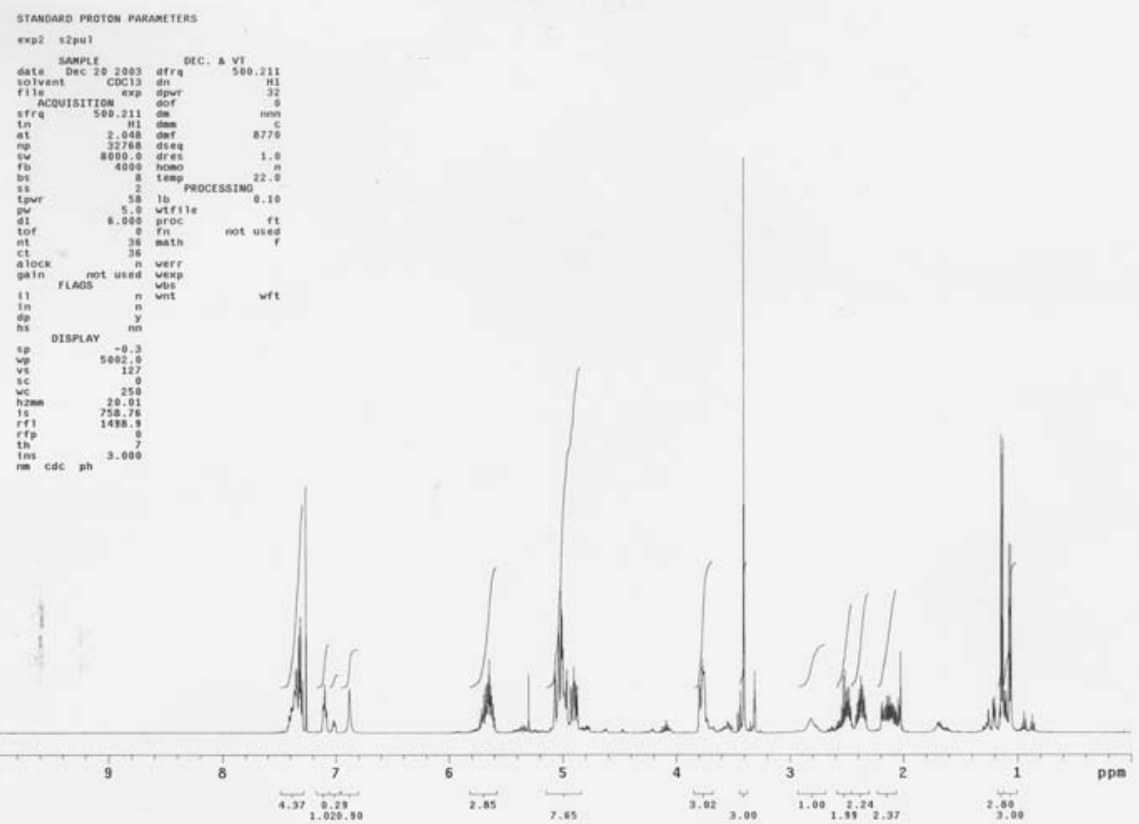

${ }^{1}$ H NMR spectrum of I 12 M 


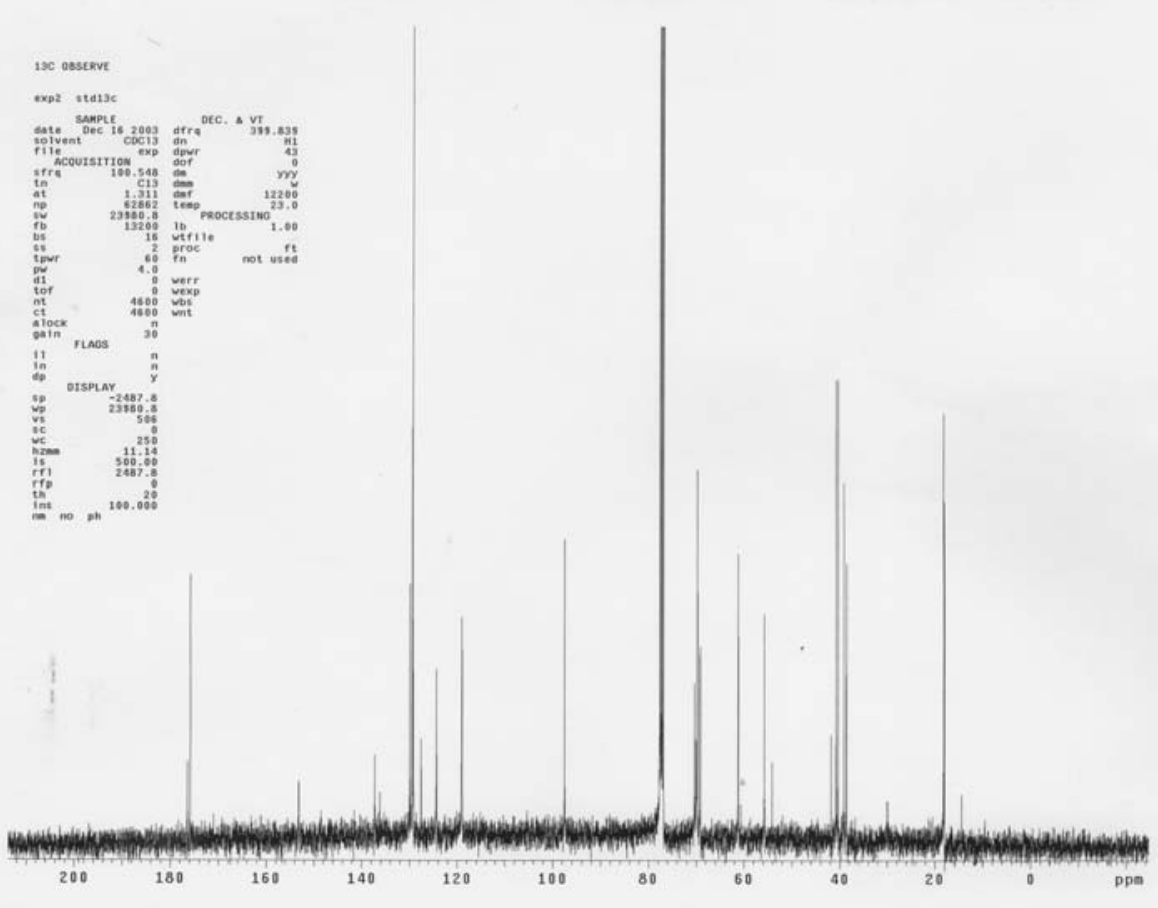

${ }^{13} \mathrm{C}$ NMR spectrum of I 12 B

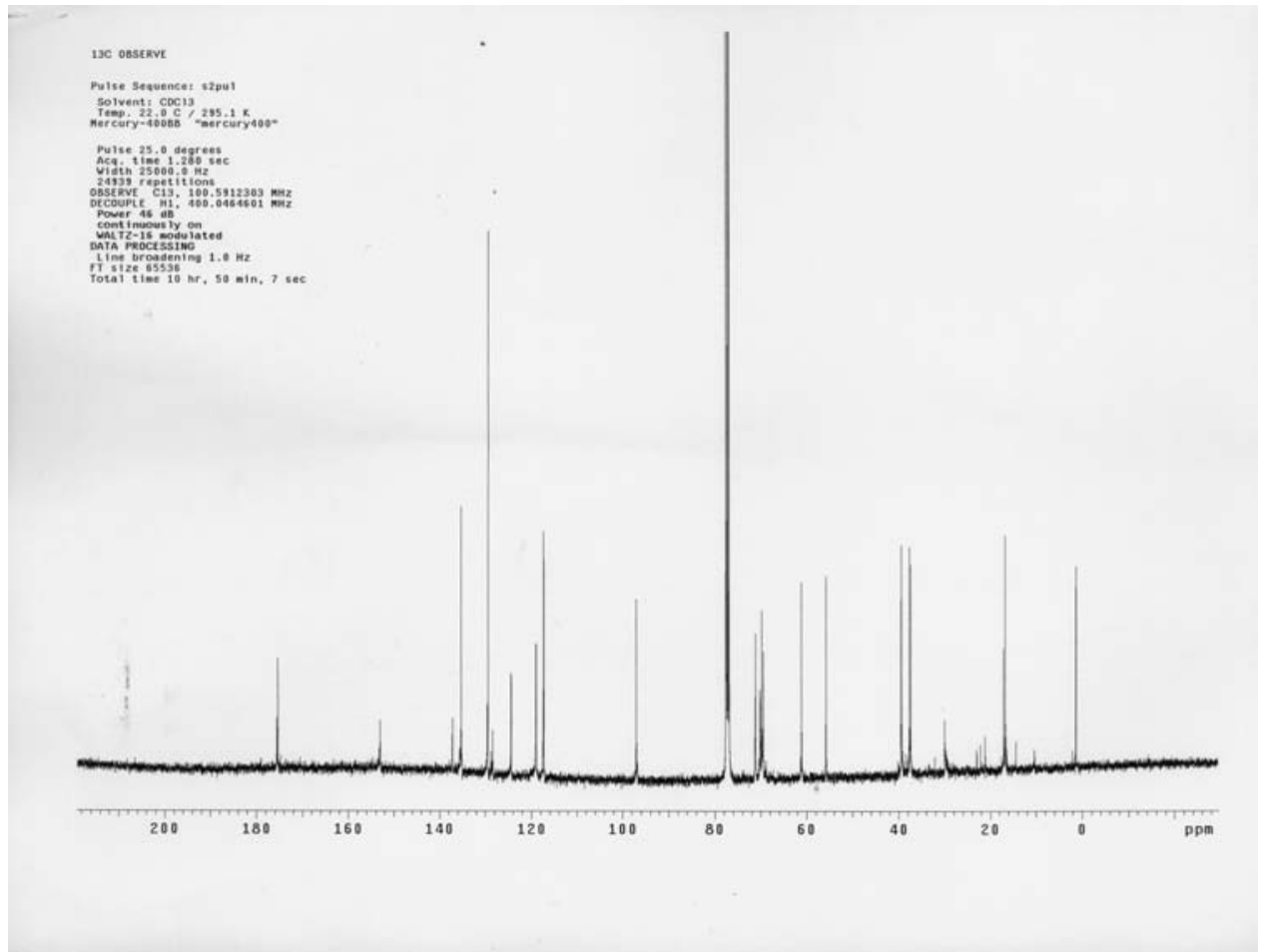

${ }^{13} \mathrm{C}$ NMR spectrum of I 12 B 


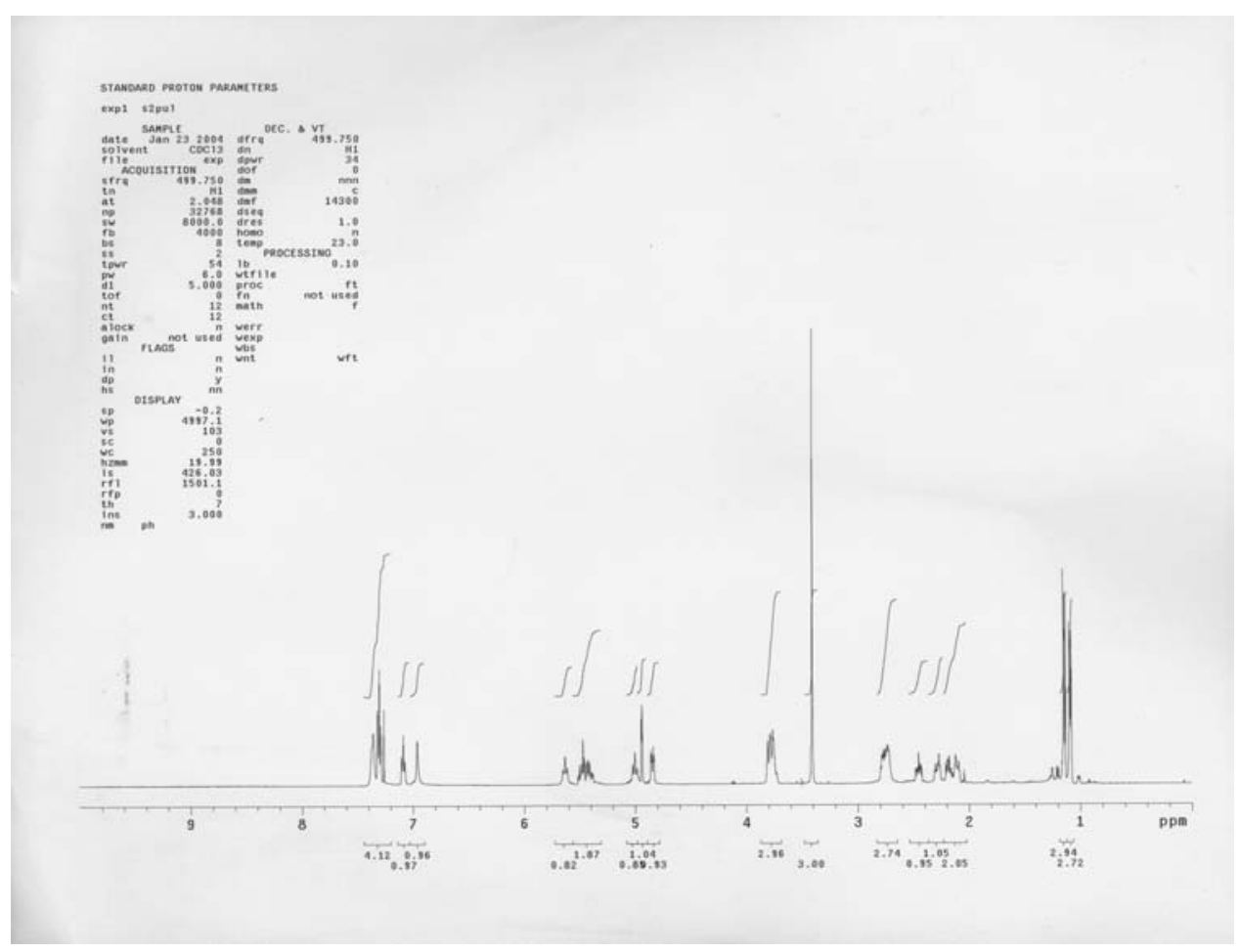

${ }^{1} \mathrm{H}$ NMR spectrum of $\left[\begin{array}{lll}1 & 1 & 3\end{array}\right]$

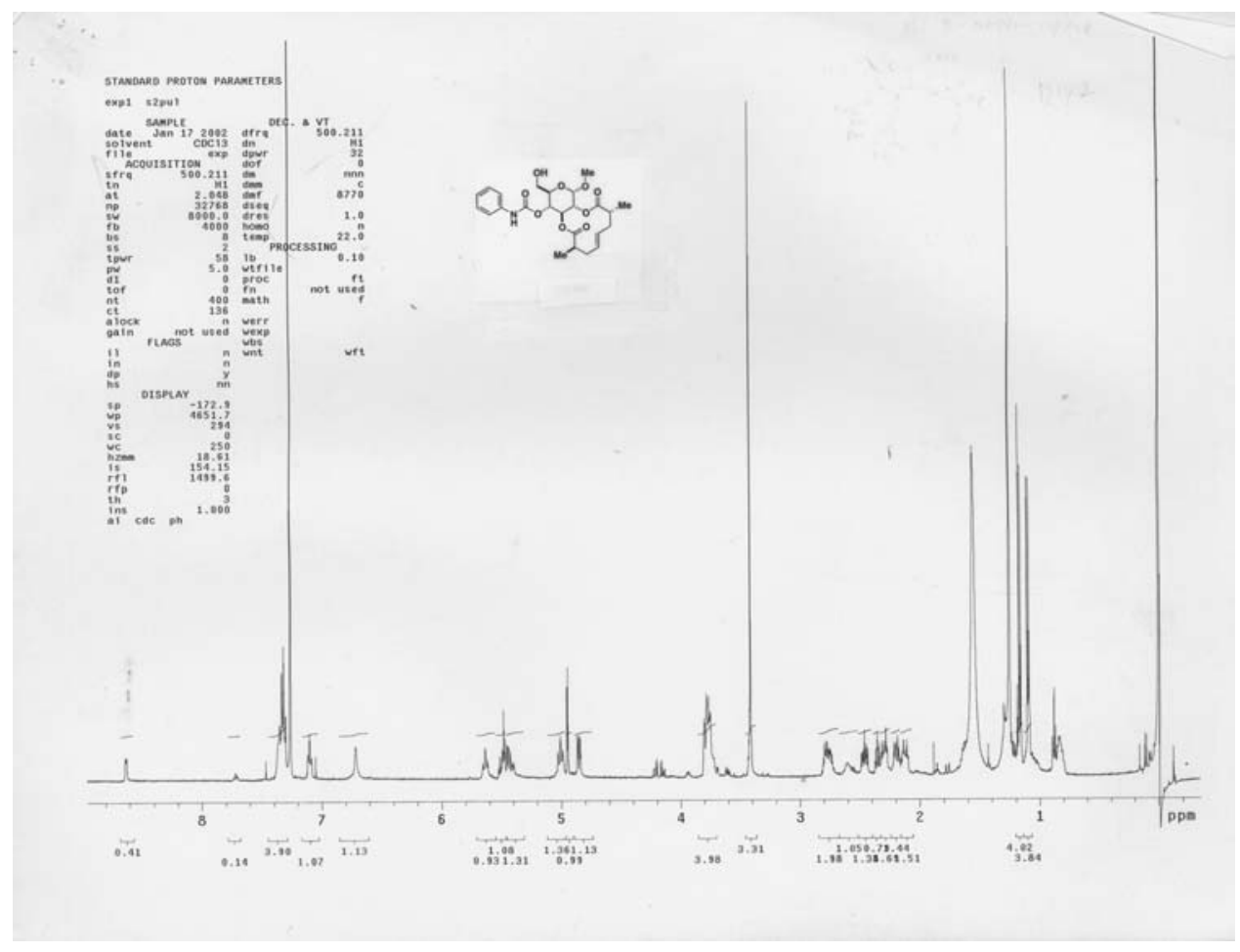

${ }^{1} \mathrm{H}$ NMR spectrum of [ [ $\left.\begin{array}{lll}1 & 3 & \text { B }\end{array}\right]$ without purification 
STANOMRo Proton PARAnet TeRs

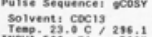

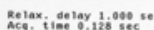

Vy

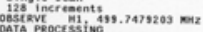

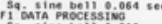

tois

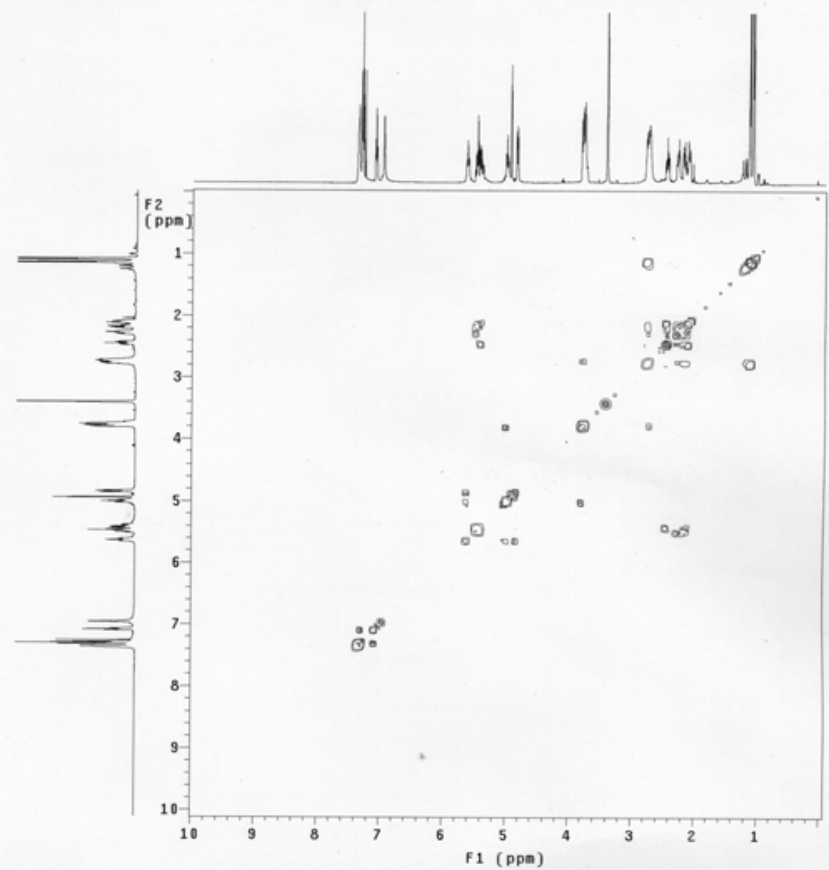

${ }^{1} \mathrm{H}$ COSY NMR spectrum of [ [ l 113 B]

$19 \mathrm{C}$ osserve

Pulse Sequencers s20ur

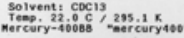

Pulse 25.0 oegrees

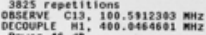

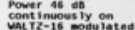

MirA reocessisito 1.0

Total tites $1 \mathrm{hr}, 57=2 \mathrm{nn}, 3 \mathrm{sec}$
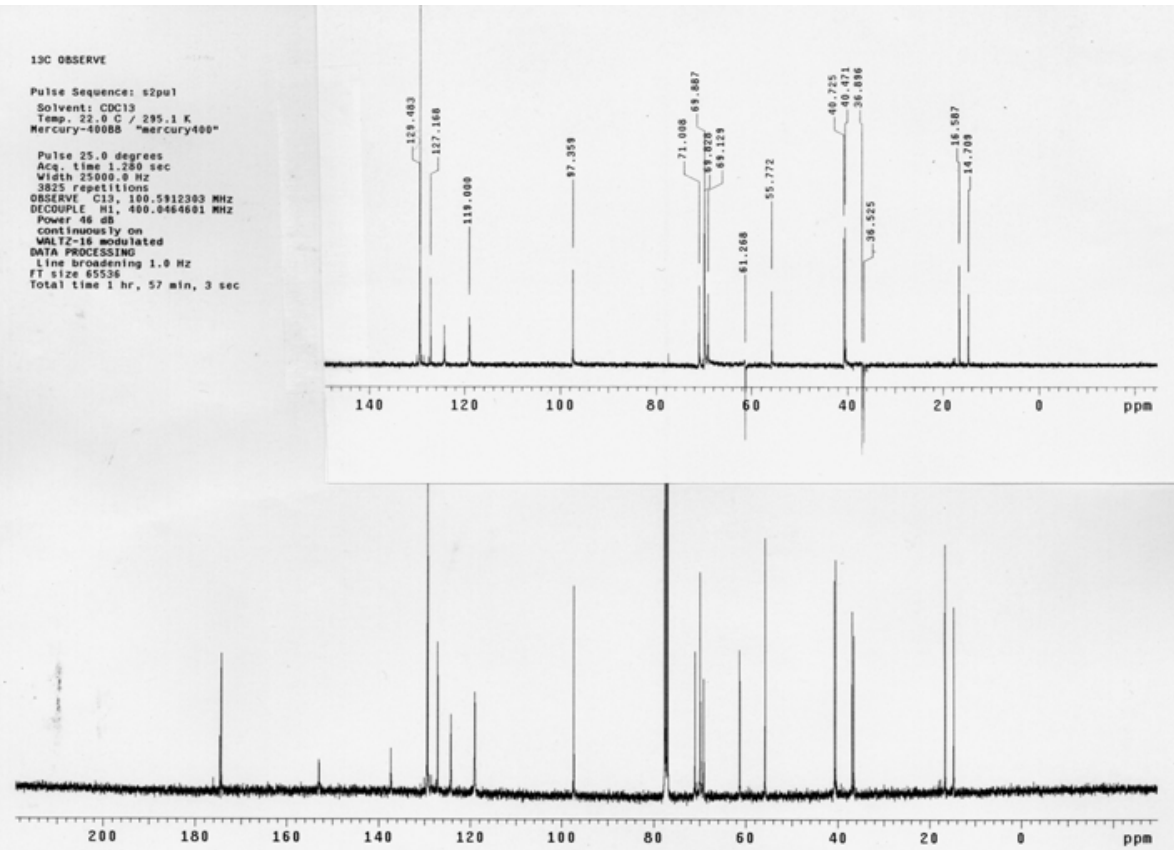

${ }^{13} \mathrm{C}$ NMR spectrum of [ [ l $113 \mathrm{~B}$ B] 


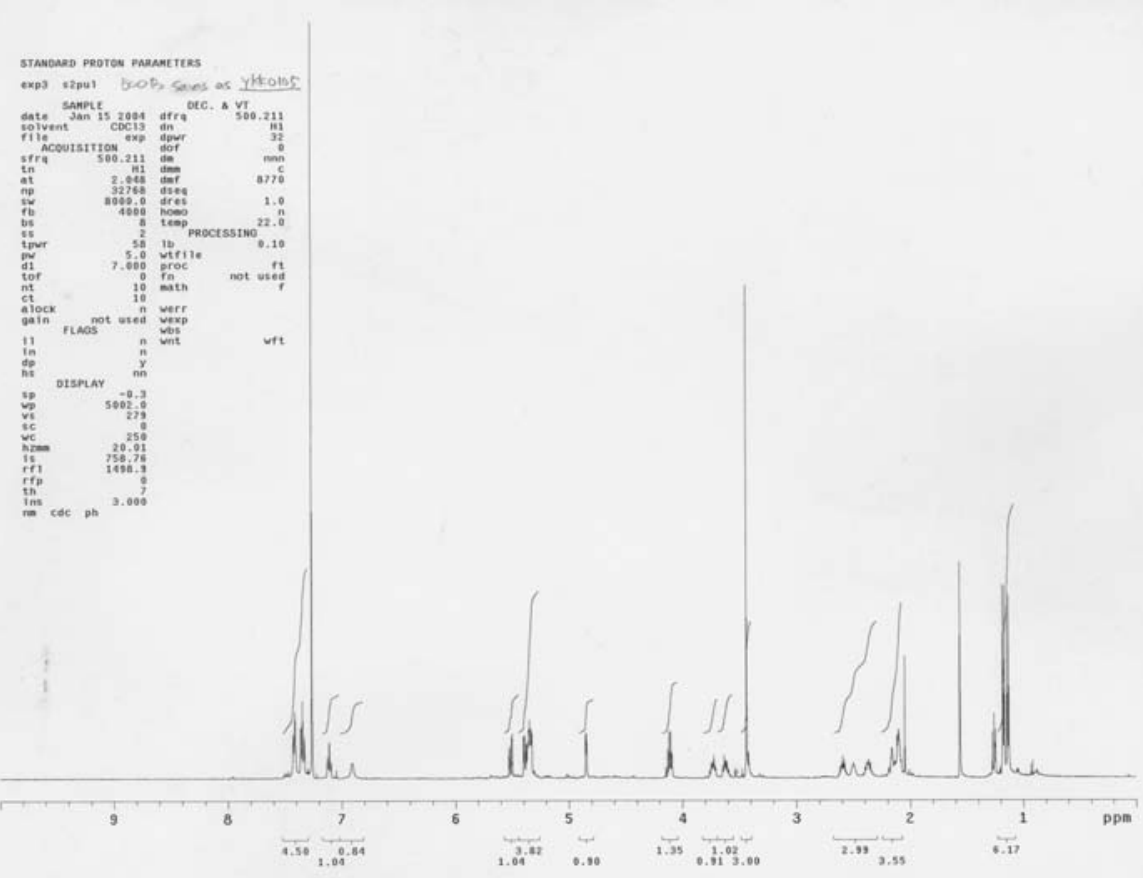

${ }^{1}$ H NMR spectrum of [II 12 B]

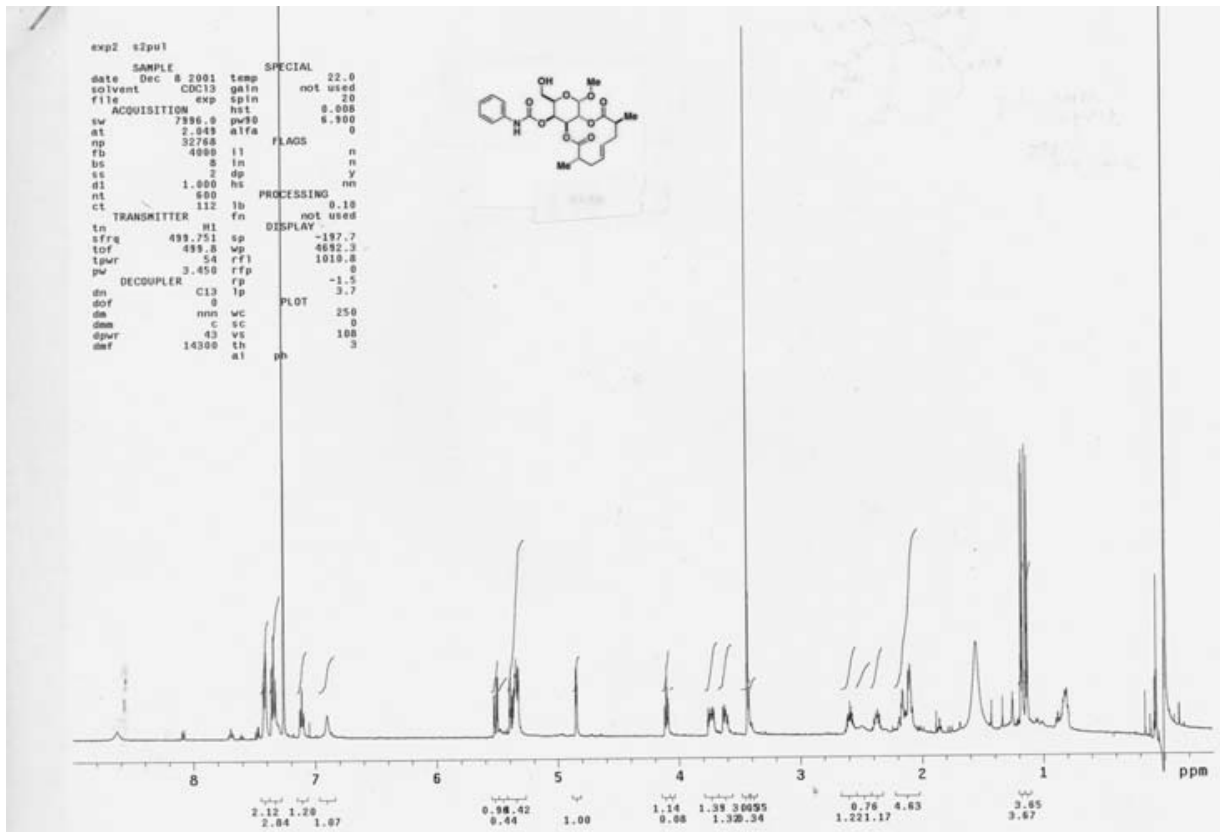

${ }^{1}$ H NMR spectrum of of [II 12 B] without purification 


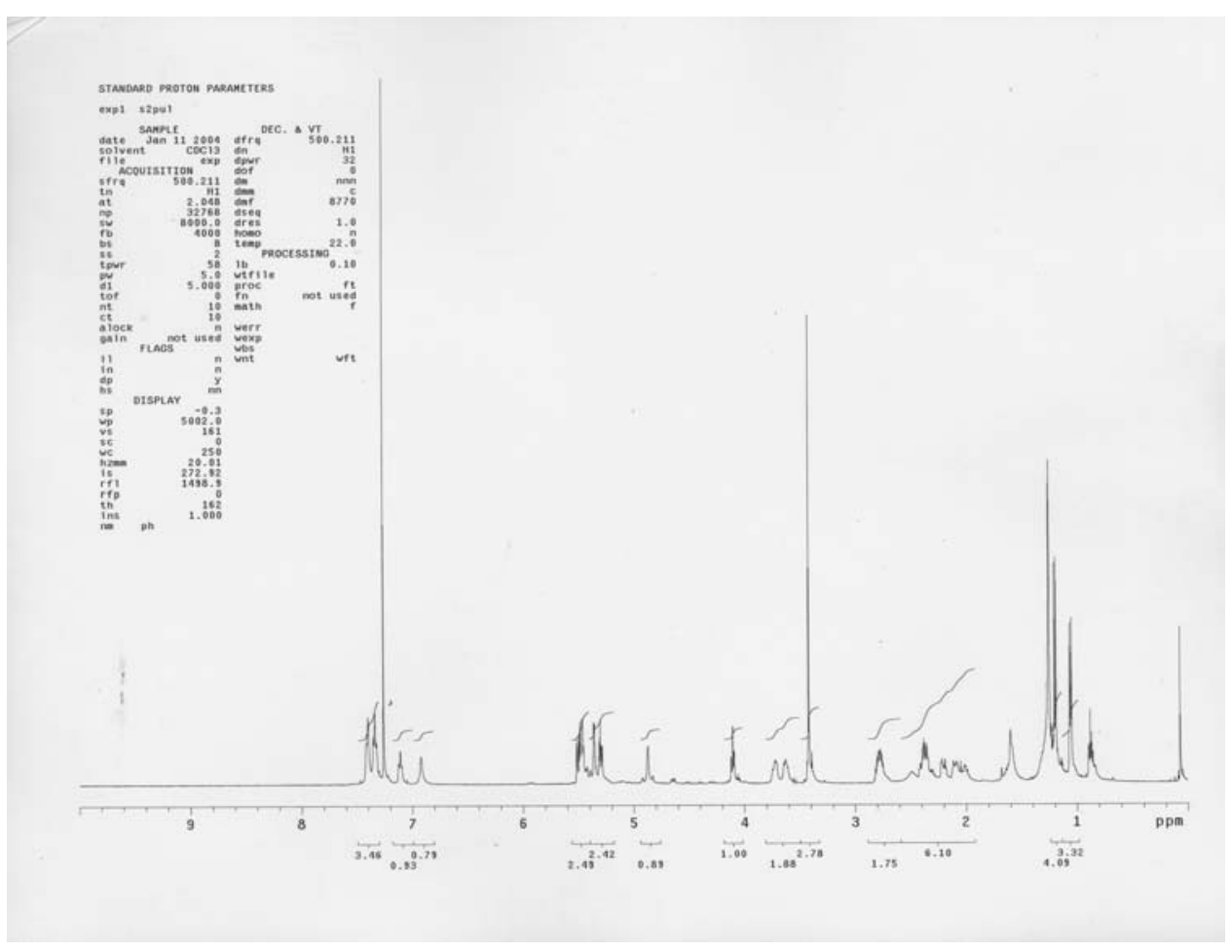

${ }^{1}$ H NMR spectrum of of [lI 13 B

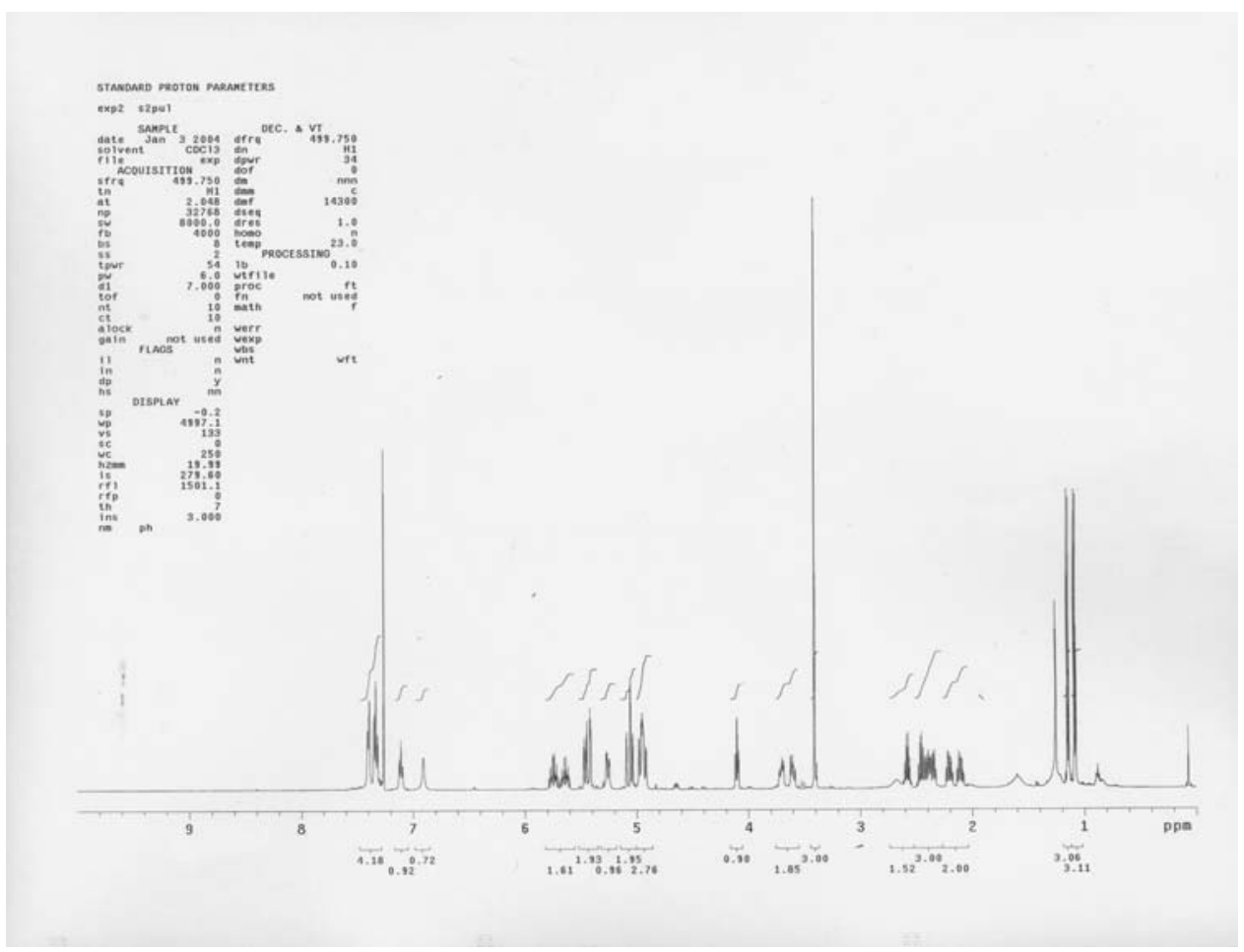

${ }^{1} \mathrm{H}$ NMR spectrum of of [II $13 \mathrm{M}$ ] 
19 osesteve

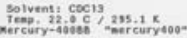

Pulas 25, aegrets

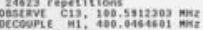

occopres mis: 460.

Sontin

tine the

sundes atos ar

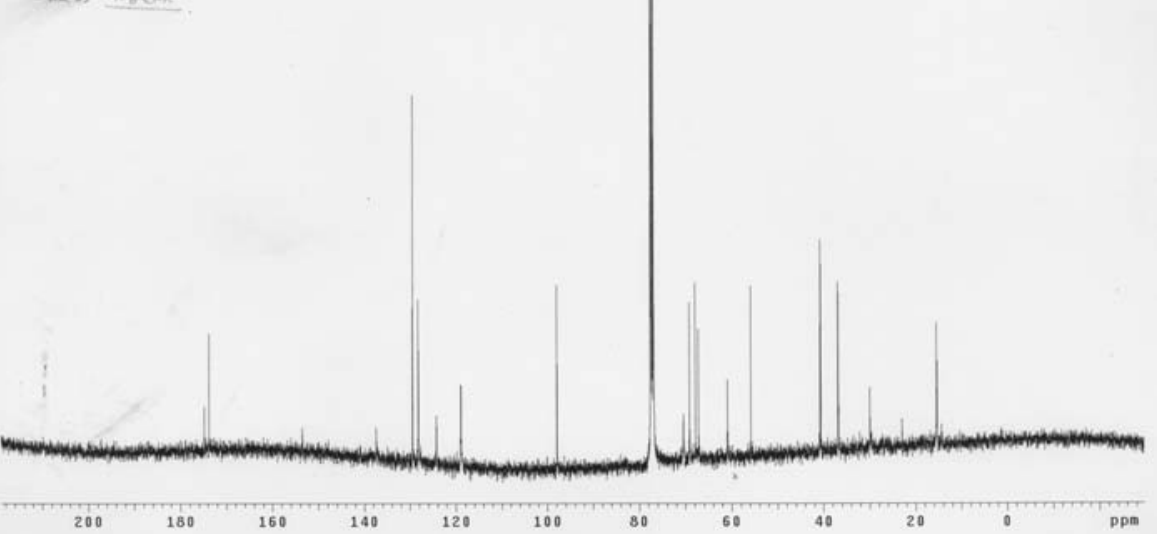

${ }^{13}$ C NMR spectrum of of [II 13 B]

136 osseave

pulse secuencen s2001

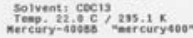

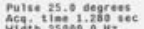

ofscove ,

cont is sin on

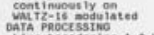

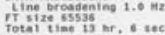

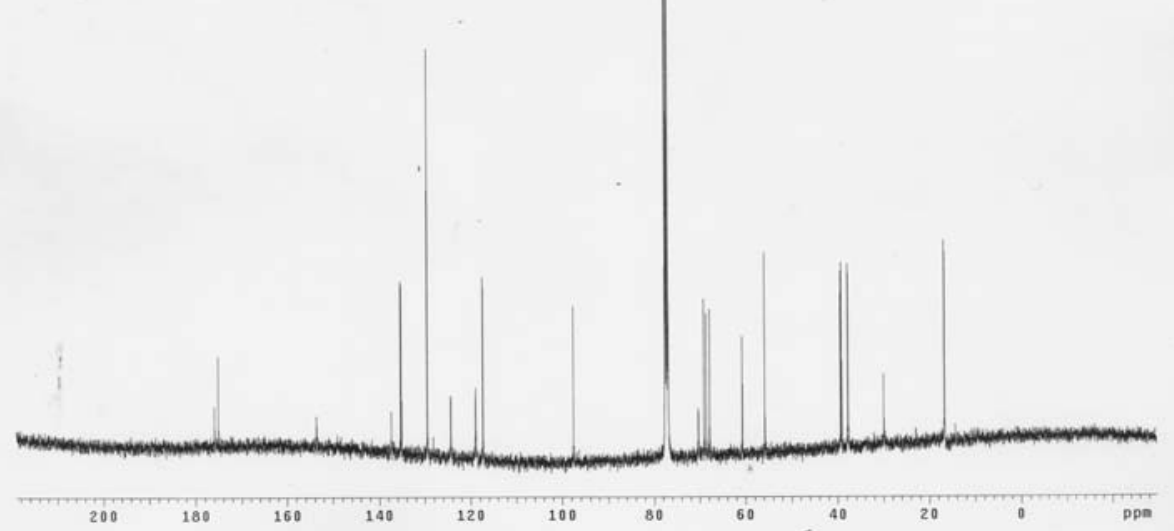

${ }^{13}$ C NMR spectrum of of [ [I 13 M] 


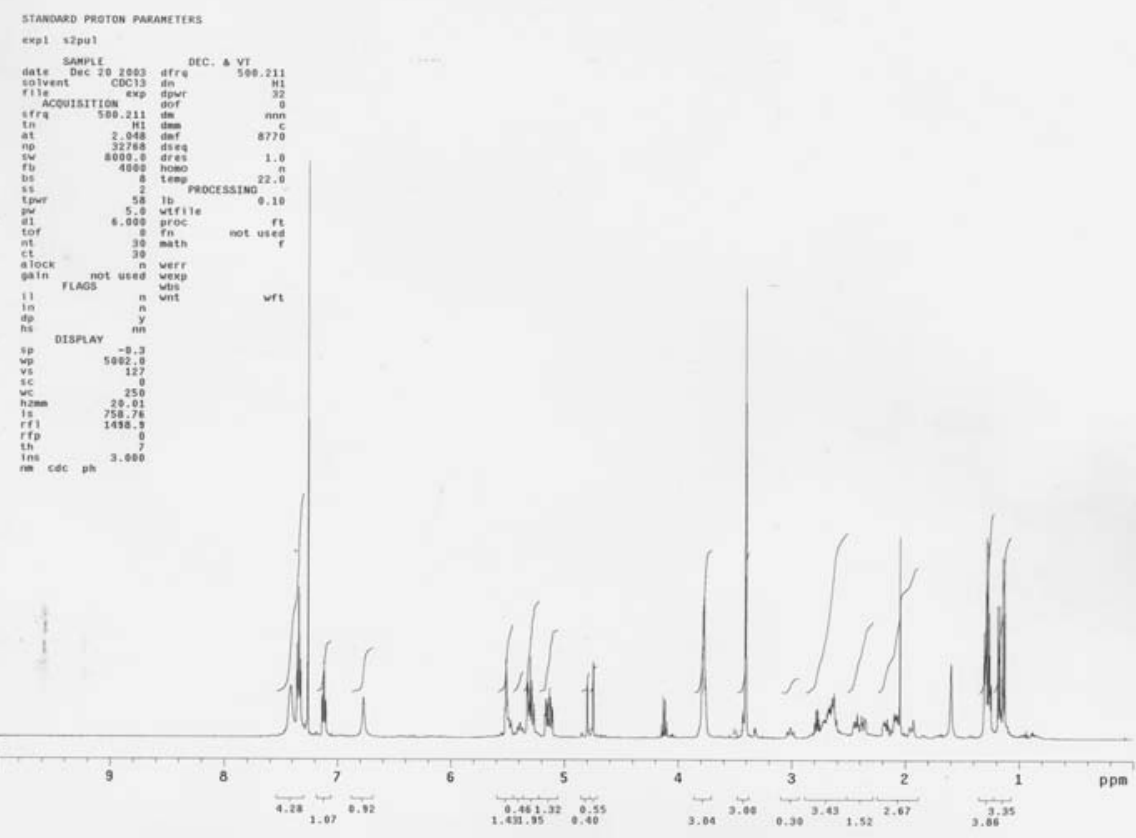

${ }^{1} \mathrm{H}$ NMR spectrum of of [III 12 B]

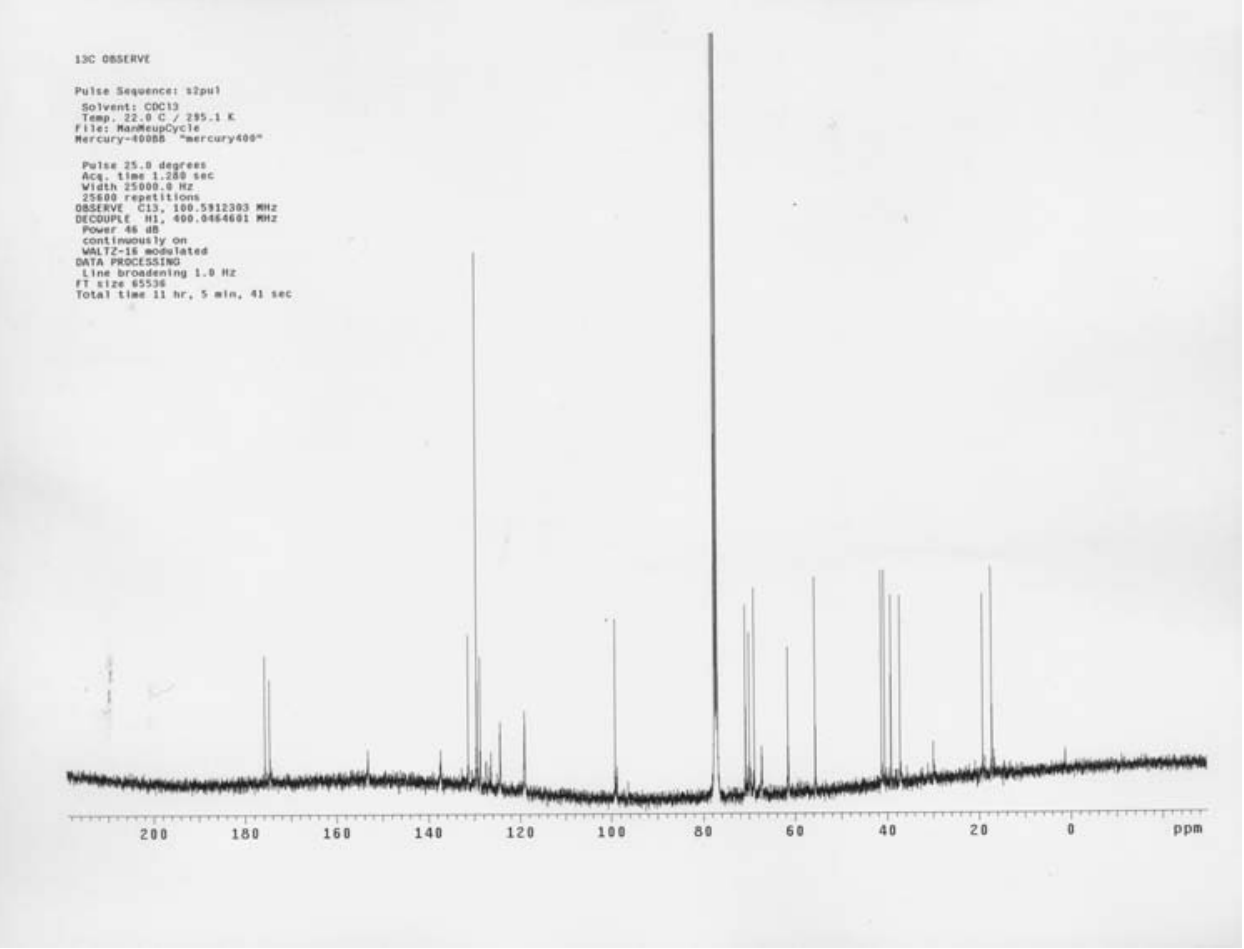

${ }^{13} \mathrm{C}$ NMR spectrum of of [III $12 \mathrm{~B}$ ] 


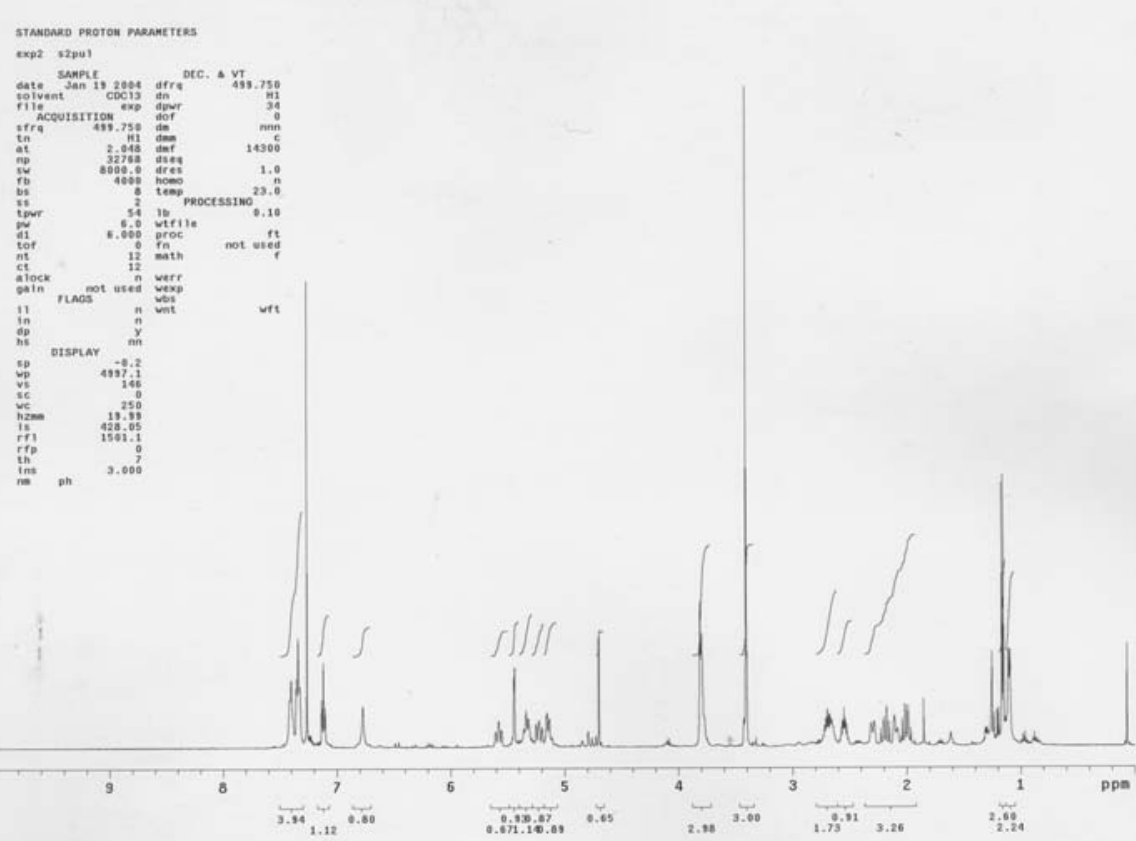

${ }^{1} \mathrm{H}$ NMR spectrum of [III 13 B]

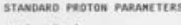

$0 \times 2$ sapul

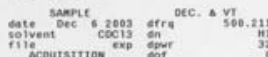

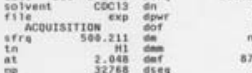

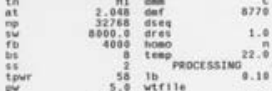

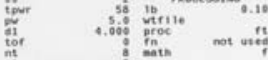

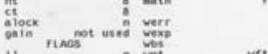

II naos

otsplat $\quad$ sooa.:

iii

I.

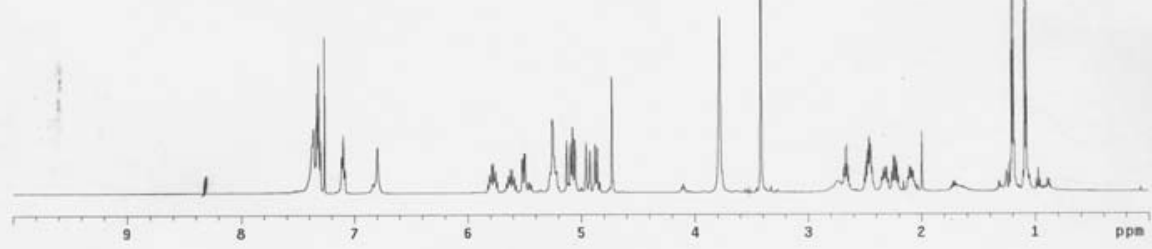

${ }^{1}$ H NMR spectrum of [III 13 M] 


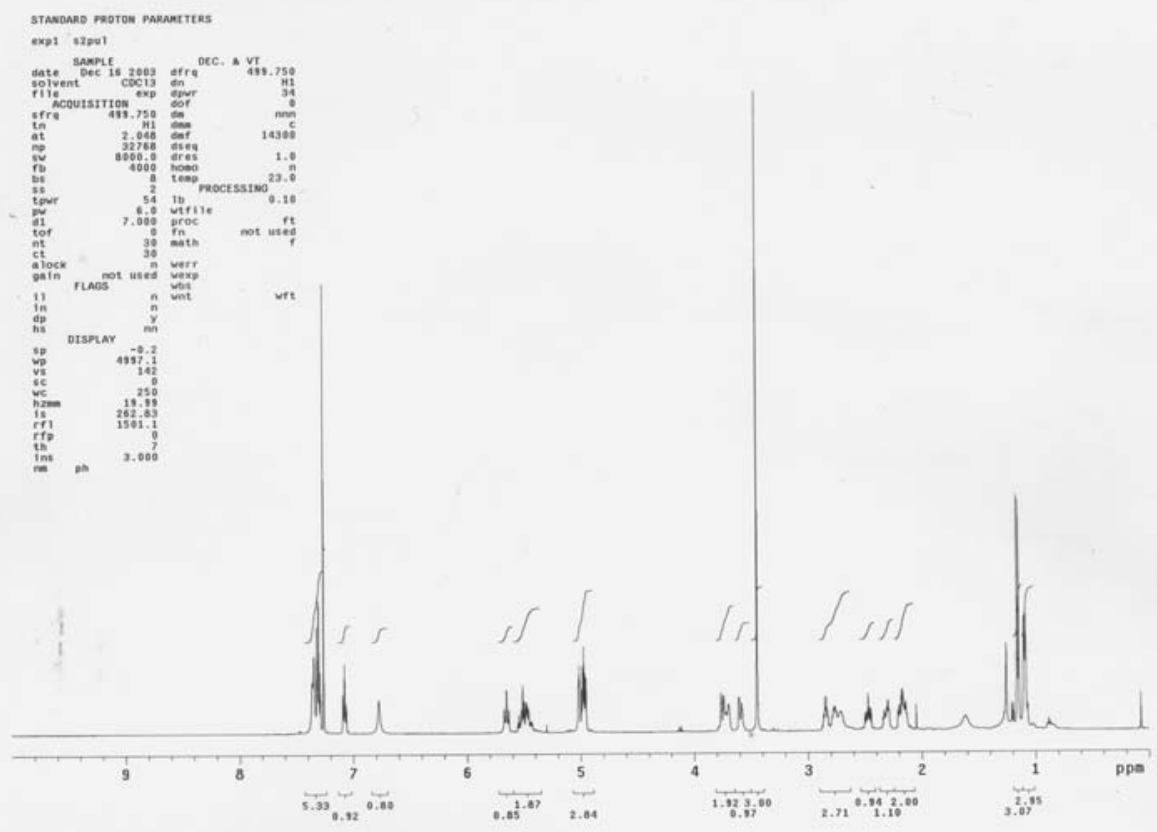

${ }^{1}$ H NMR spectrum of [IV 12 B]

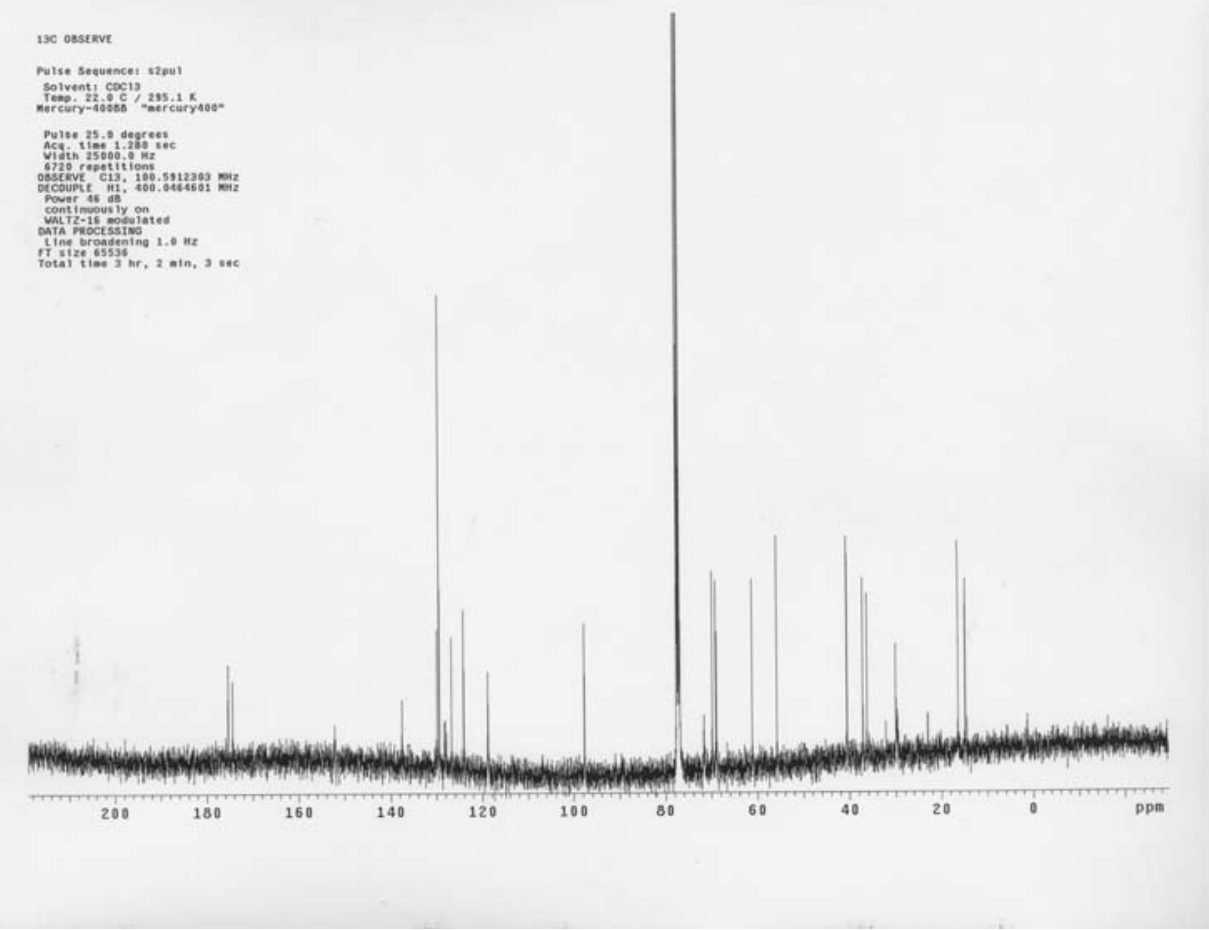

${ }^{13} \mathrm{C}$ NMR spectrum of [IV 12 B] 


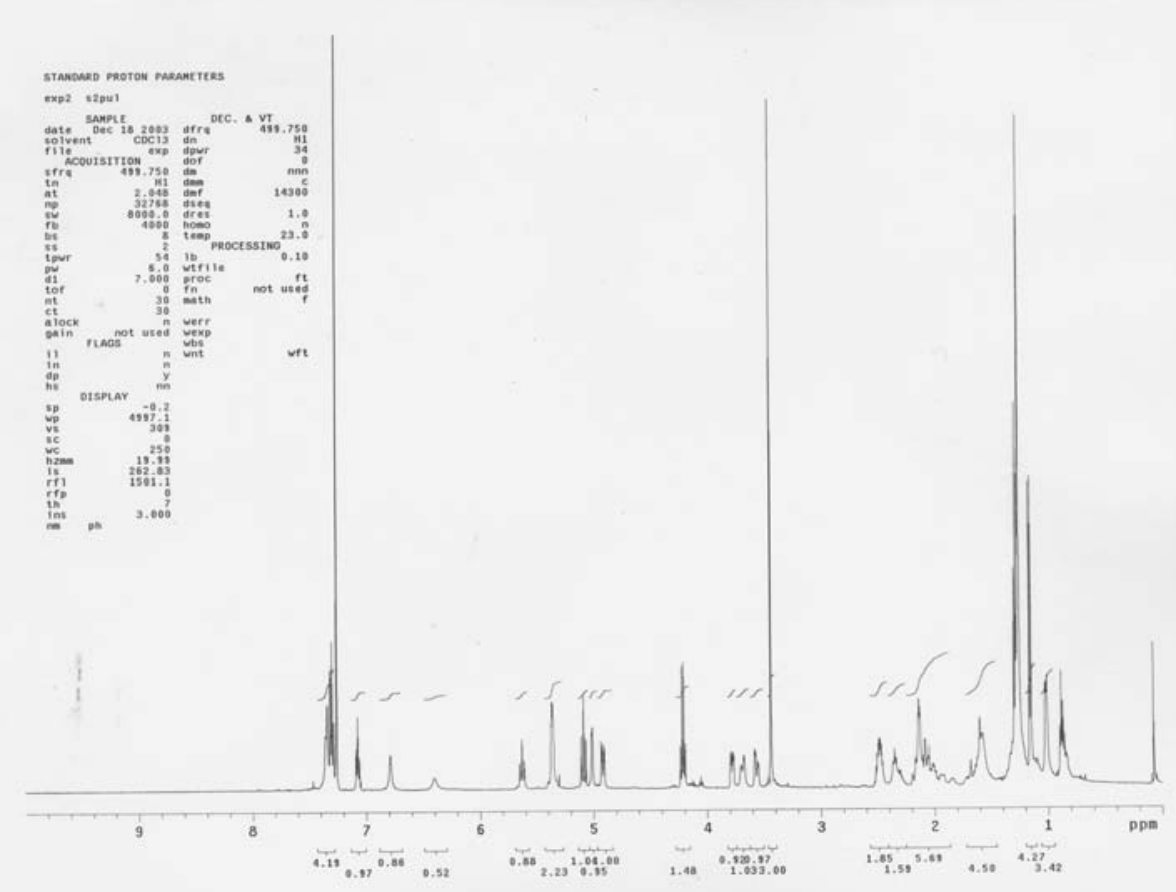

${ }^{1}$ H NMR spectrum of [IV 13 B]

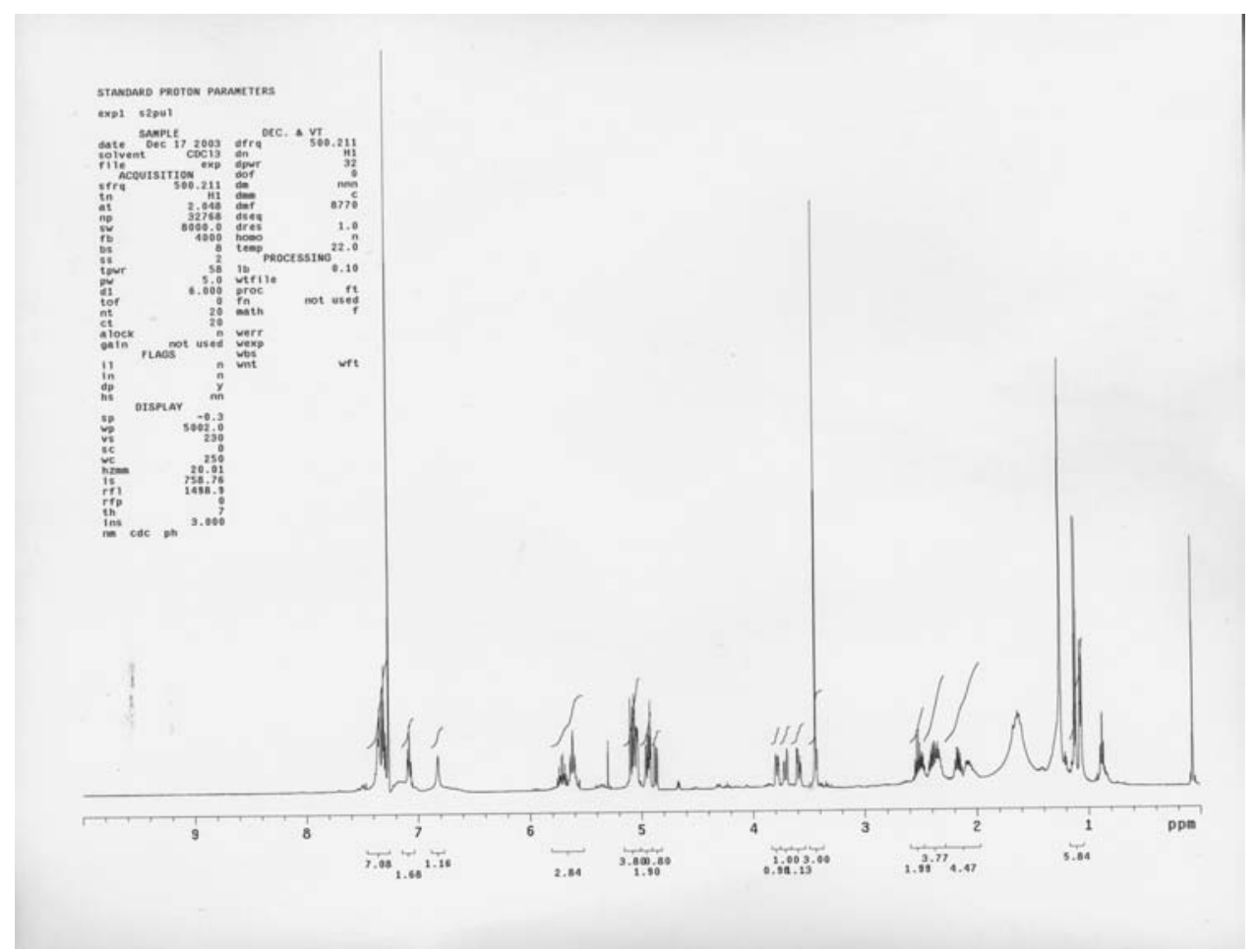

${ }^{1} \mathrm{H}$ NMR spectrum of [IV $13 \mathrm{M}$ ] 
stancasp proton meantres:

expl szovi

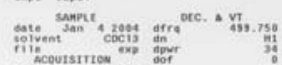

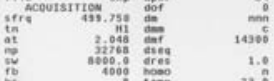

tit $\quad 4000$ the

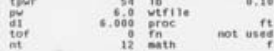

ciock 12 vert

11 rasot used vexp

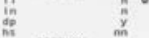

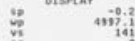

空

落

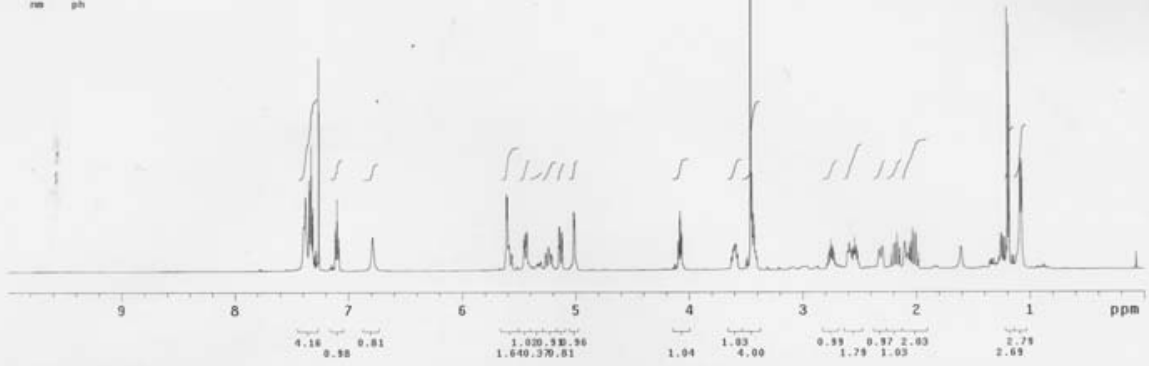

${ }^{1} \mathrm{H}$ NMR spectrum of [ [ 12 ( 2 B]

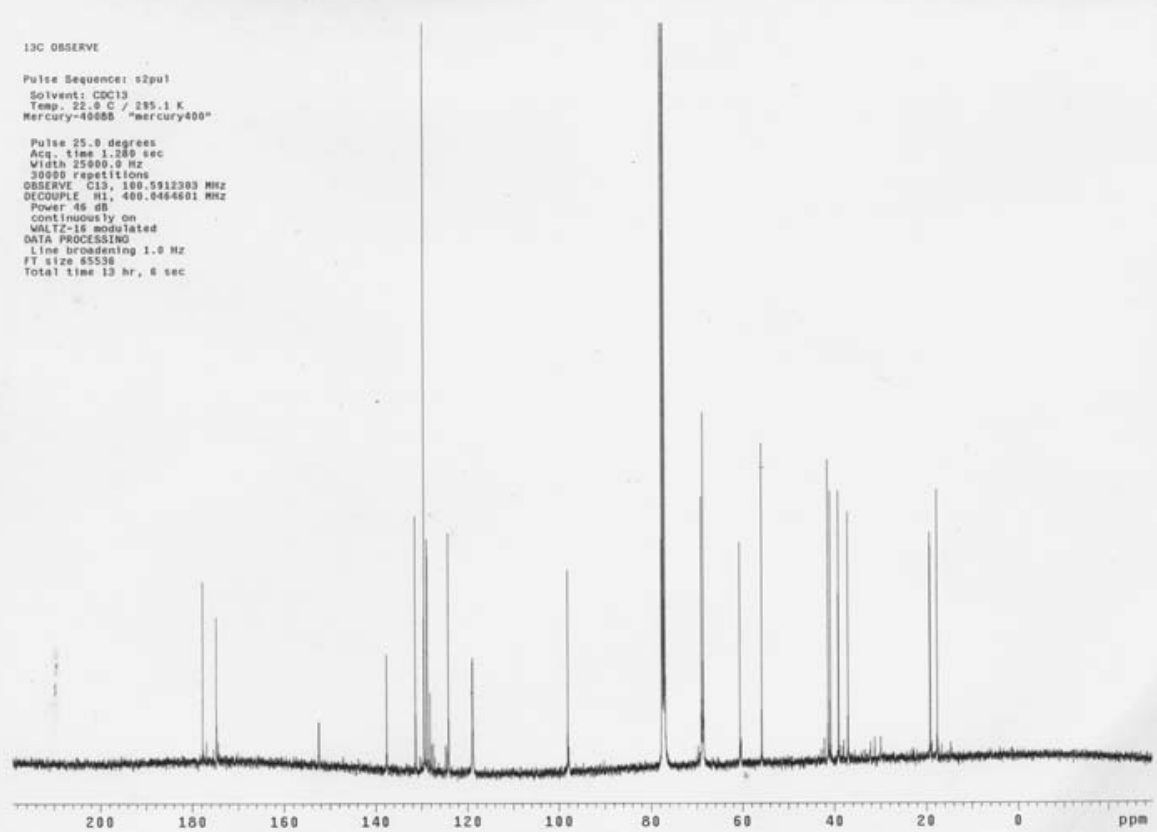

${ }^{13} \mathrm{C}$ NMR spectrum of [V $\left.112 \mathrm{~B}\right]$ 


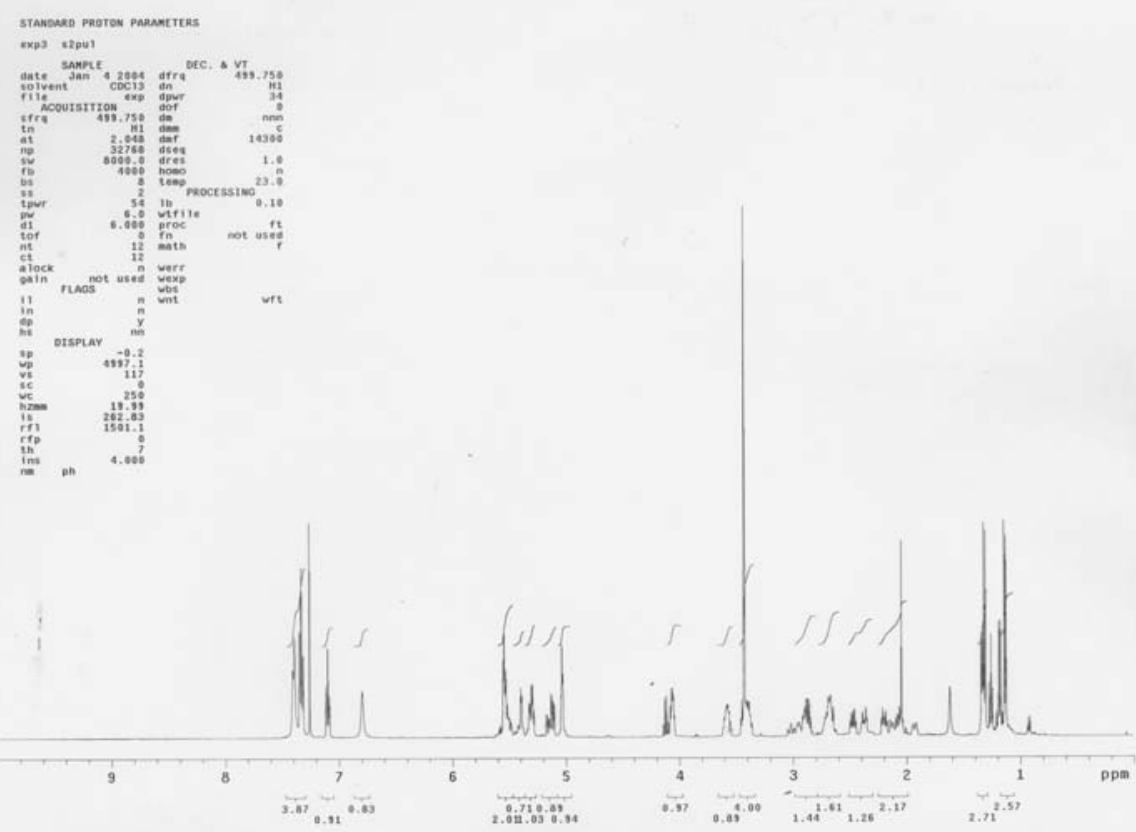

${ }^{1} \mathrm{H}$ NMR spectrum of [V 13 B]

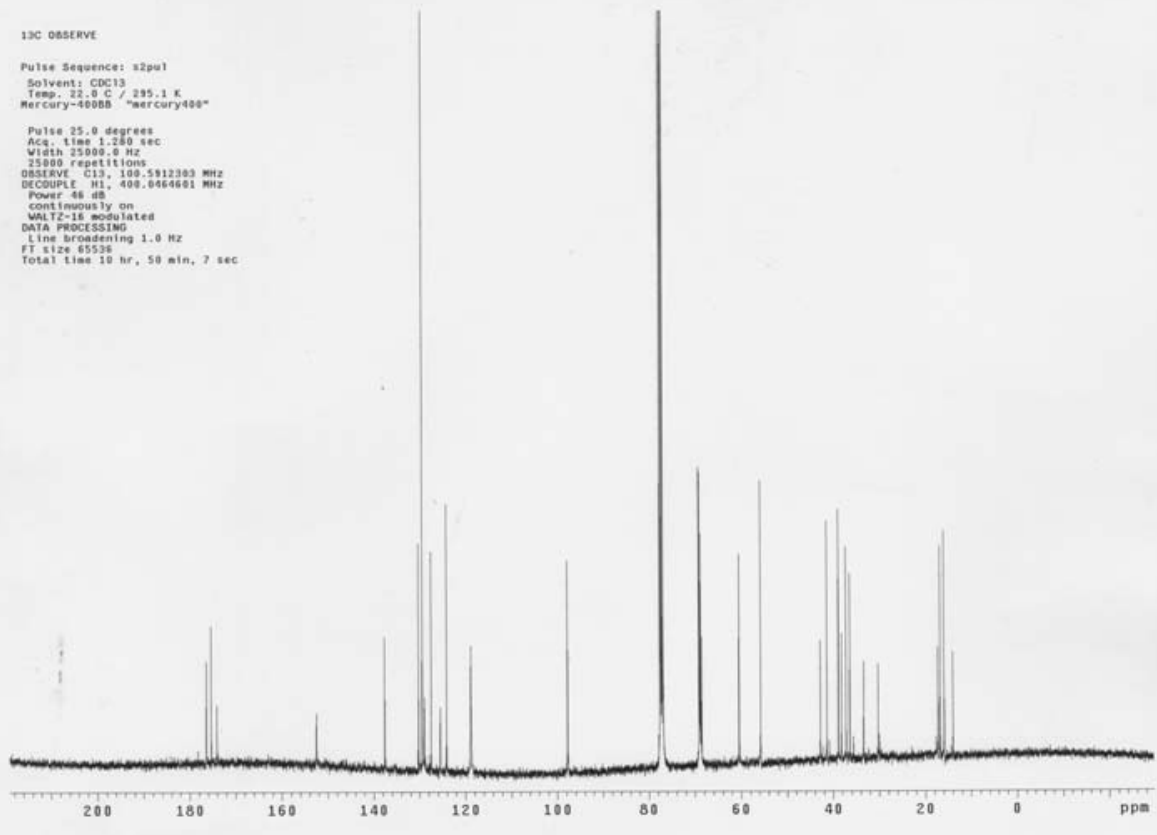

${ }^{13} \mathrm{C}$ NMR spectrum of [V 13 B] 

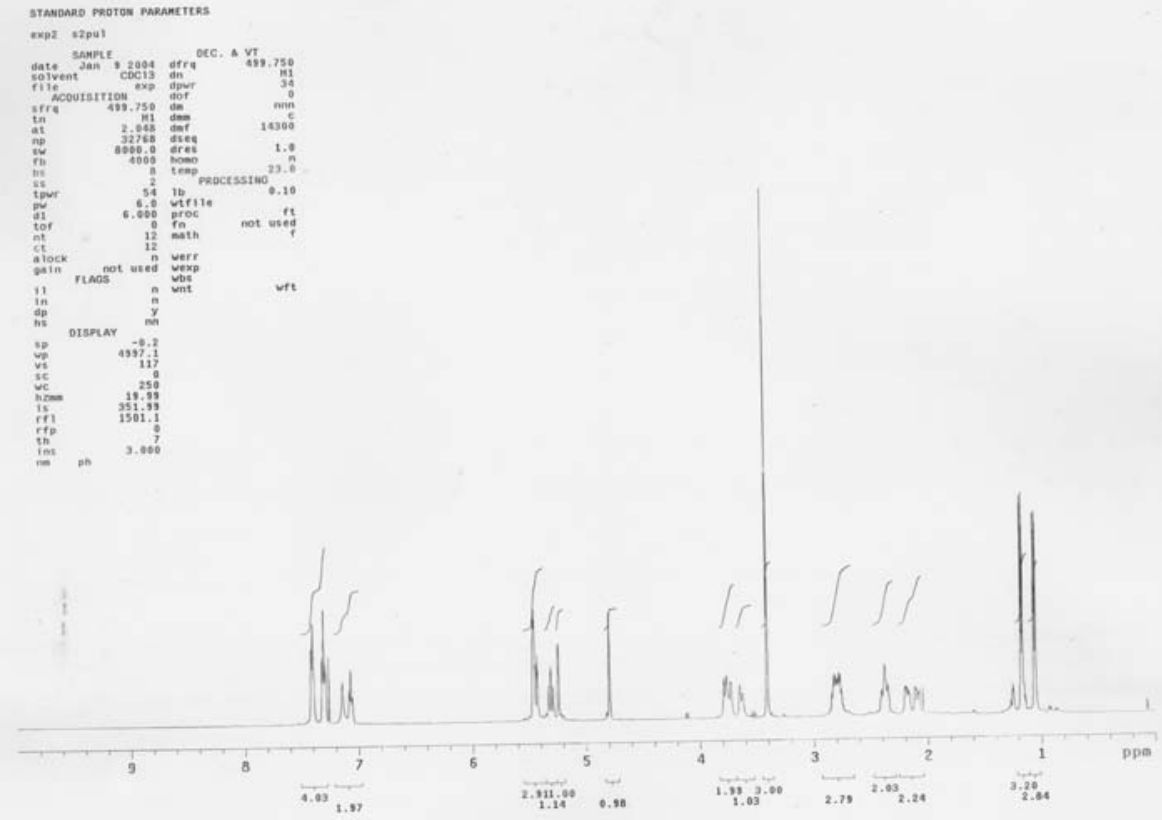

${ }^{1}$ H NMR spectrum of [VI 12 B]

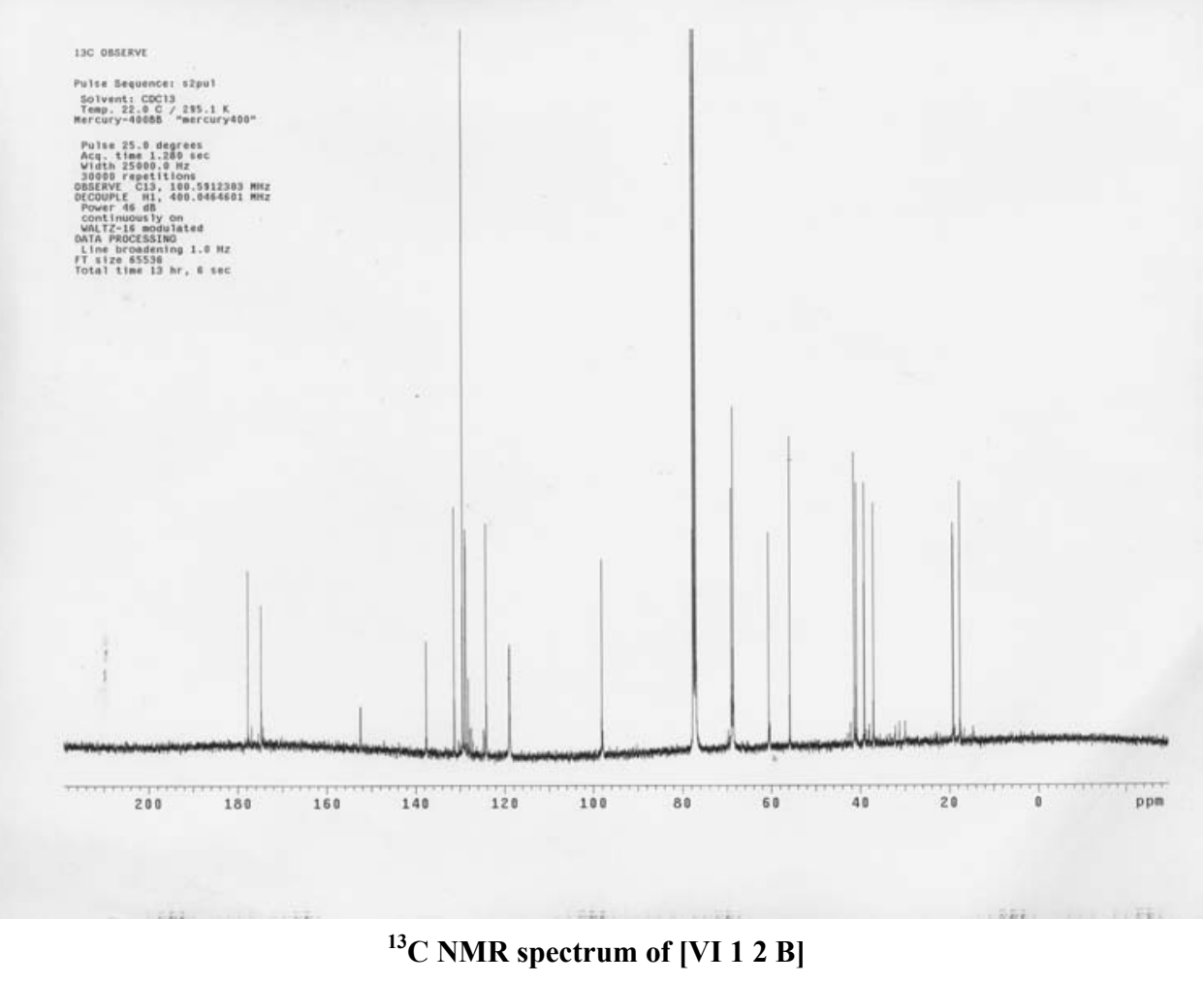




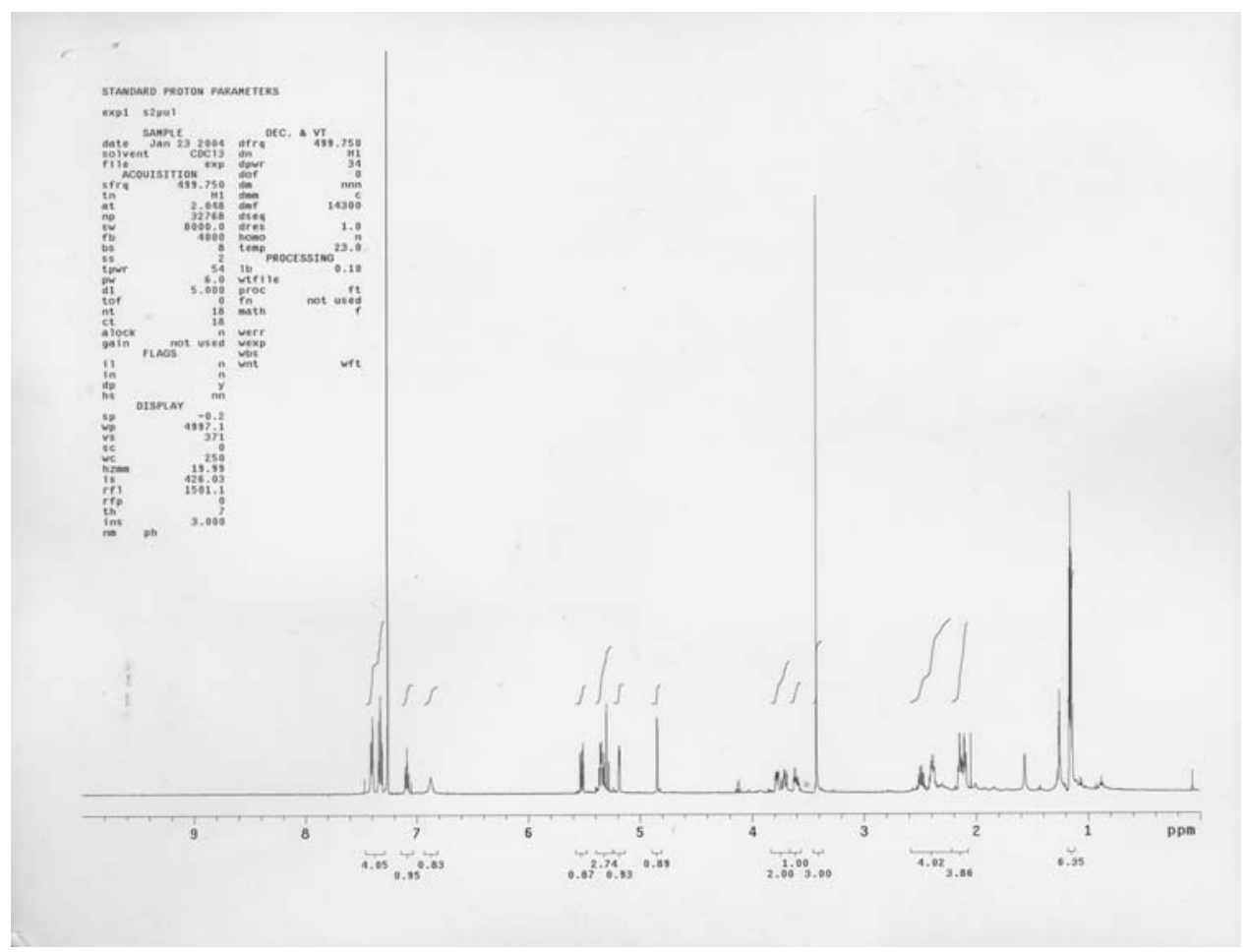

${ }^{1}$ H NMR spectrum of [VI 13 B]

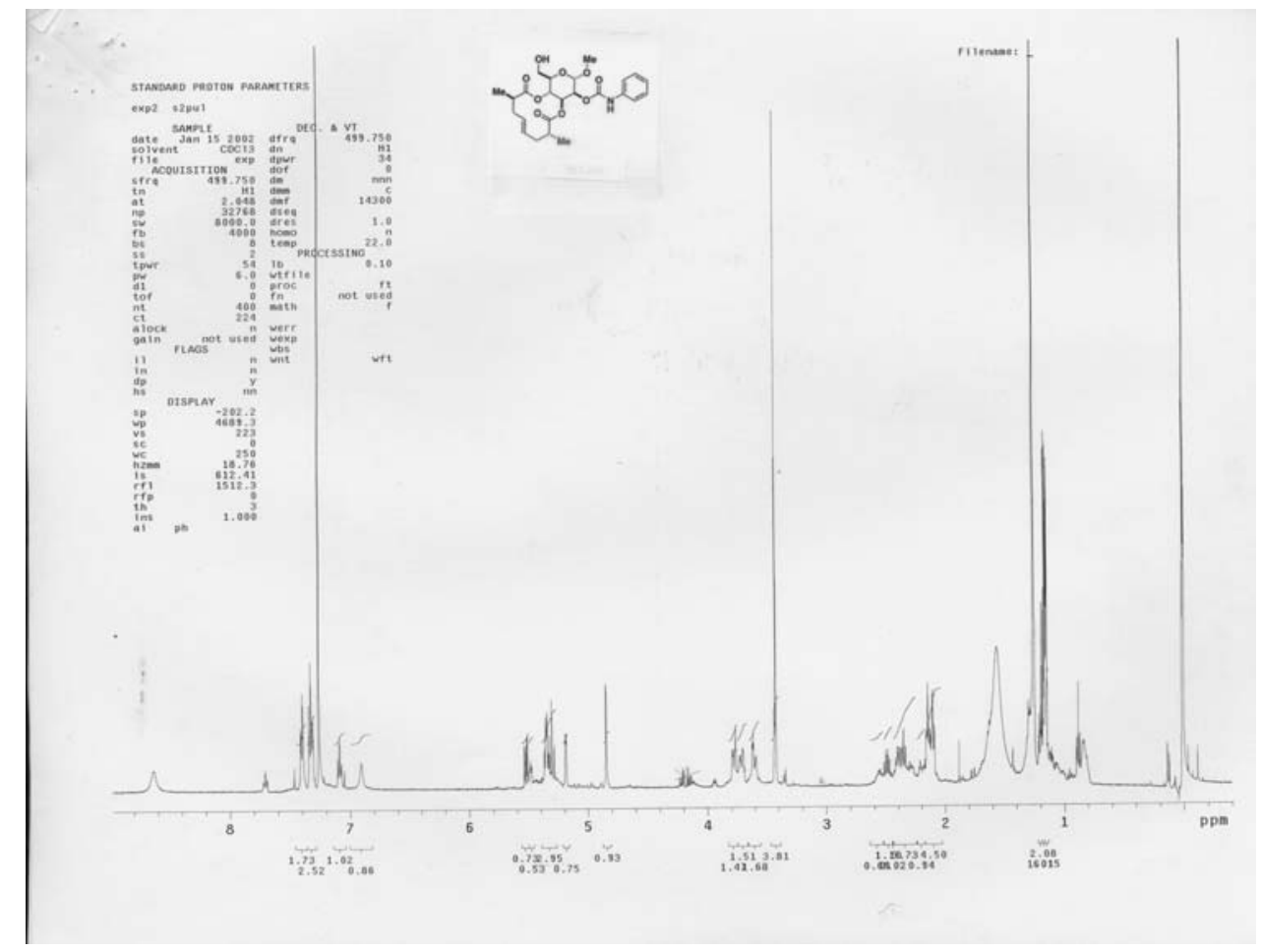

${ }^{1} \mathrm{H}$ NMR spectrum of [VI 13 B] without purification 


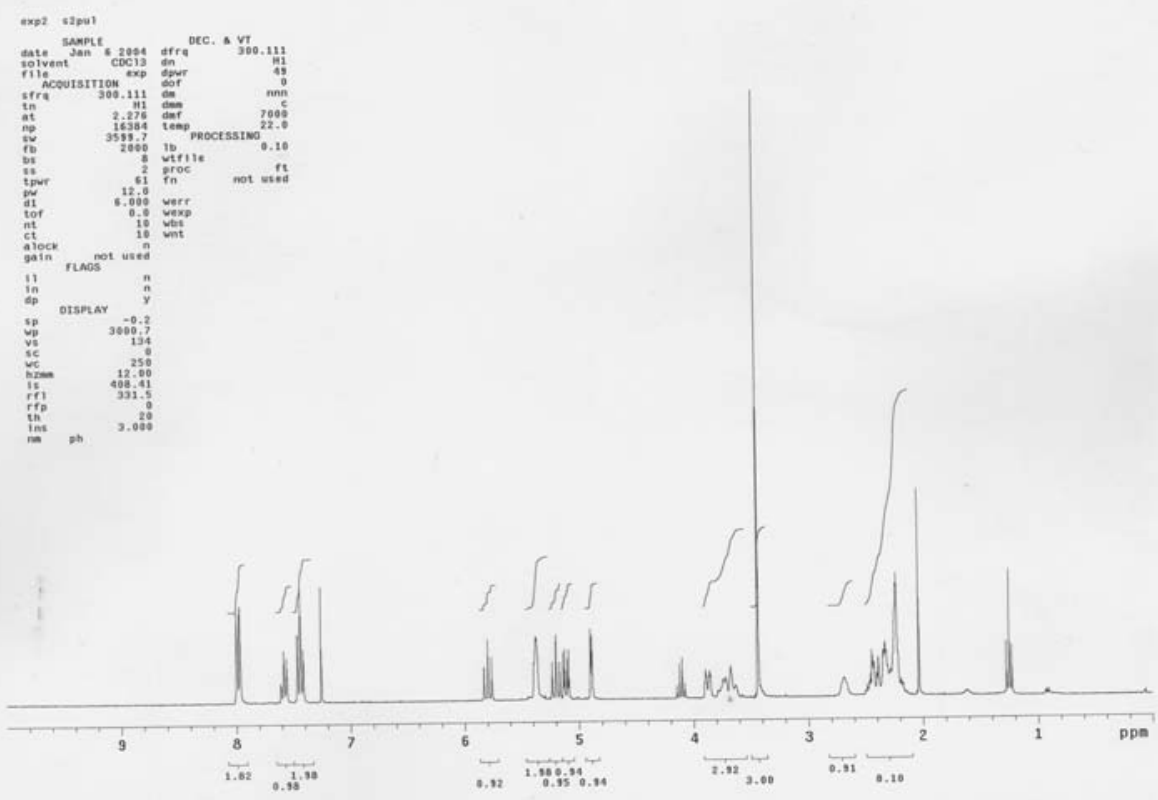

${ }^{1}$ H NMR spectrum of [l $\left.\begin{array}{lll}2 & 1 & B\end{array}\right]$

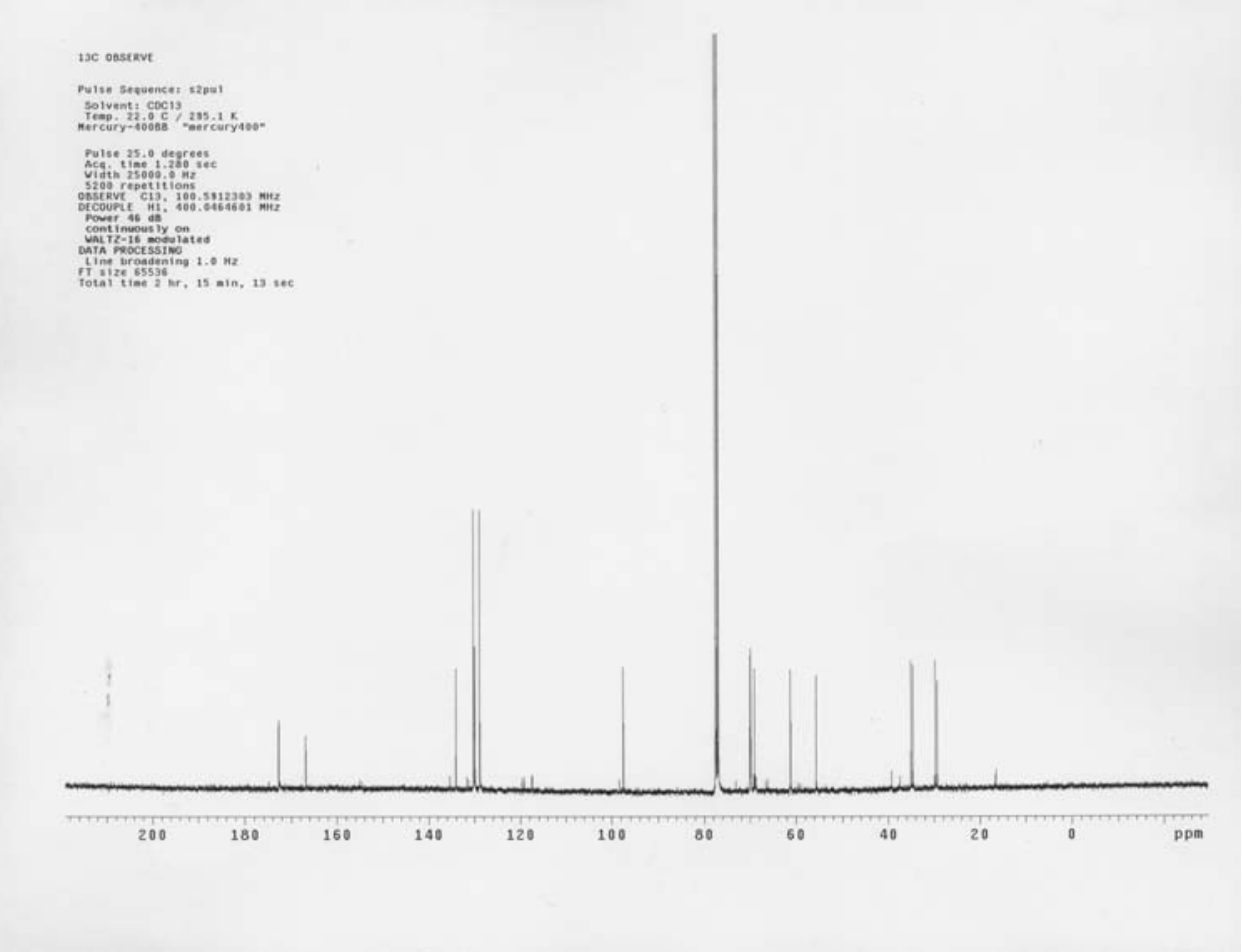

${ }^{13}$ C NMR spectrum of [I 2 1 B] 


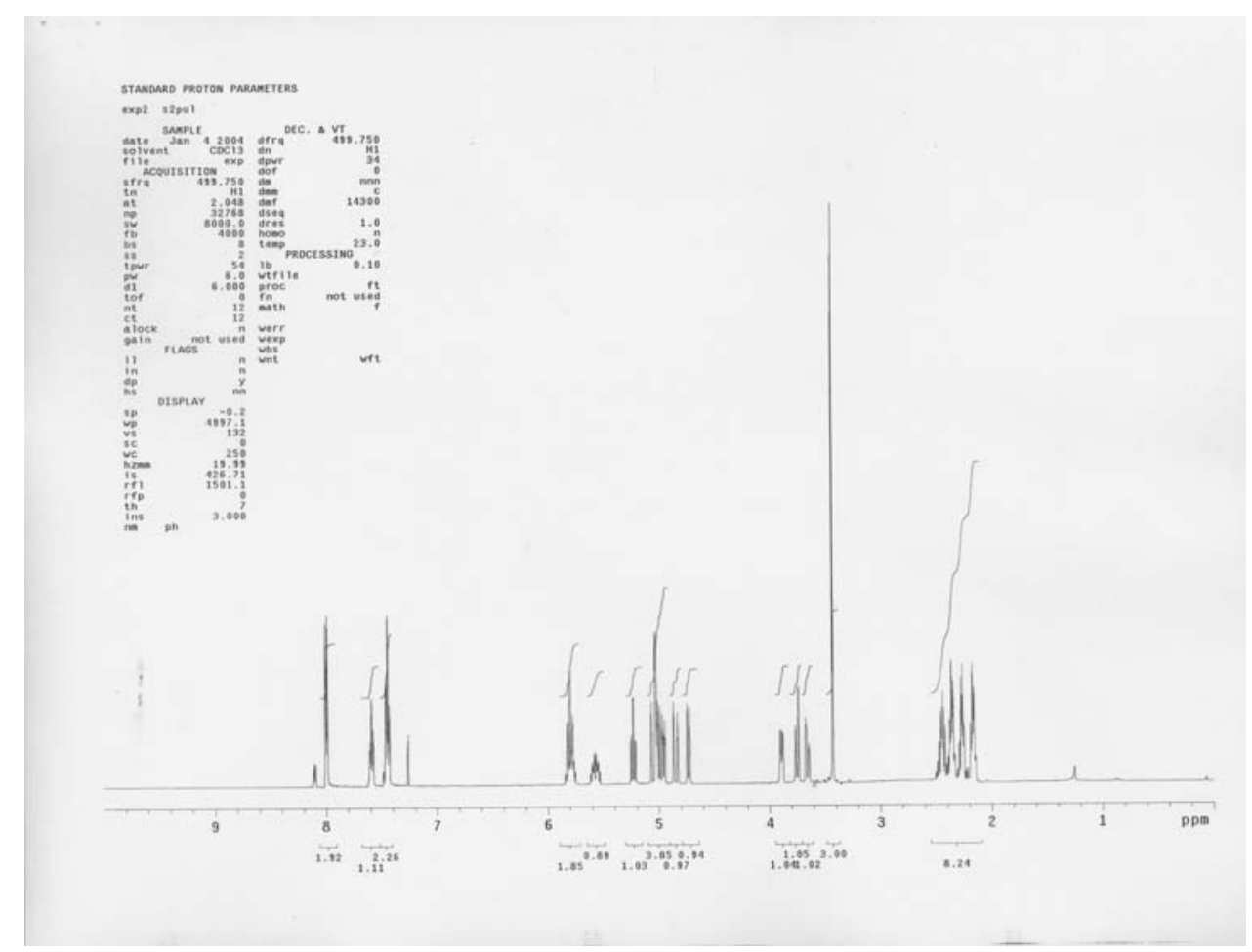

${ }^{1} \mathrm{H}$ NMR spectrum of [l $\left.2 \begin{array}{lll}2 & \text { M] }\end{array}\right]$

190 orsenve

culse Sesuencet 12001

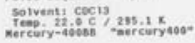

prine 25.0 asgrees.

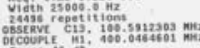

continuestily on

tithe

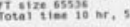

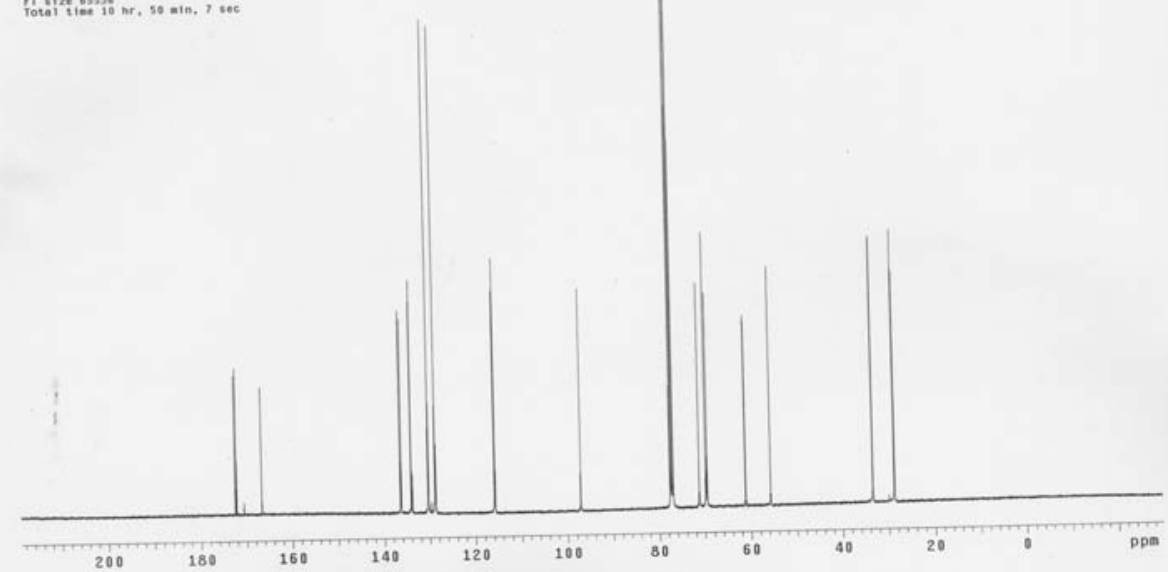

${ }^{13} \mathrm{C}$ NMR spectrum of [l $\left.\begin{array}{lll}2 & 1 & \mathrm{M}\end{array}\right]$ 


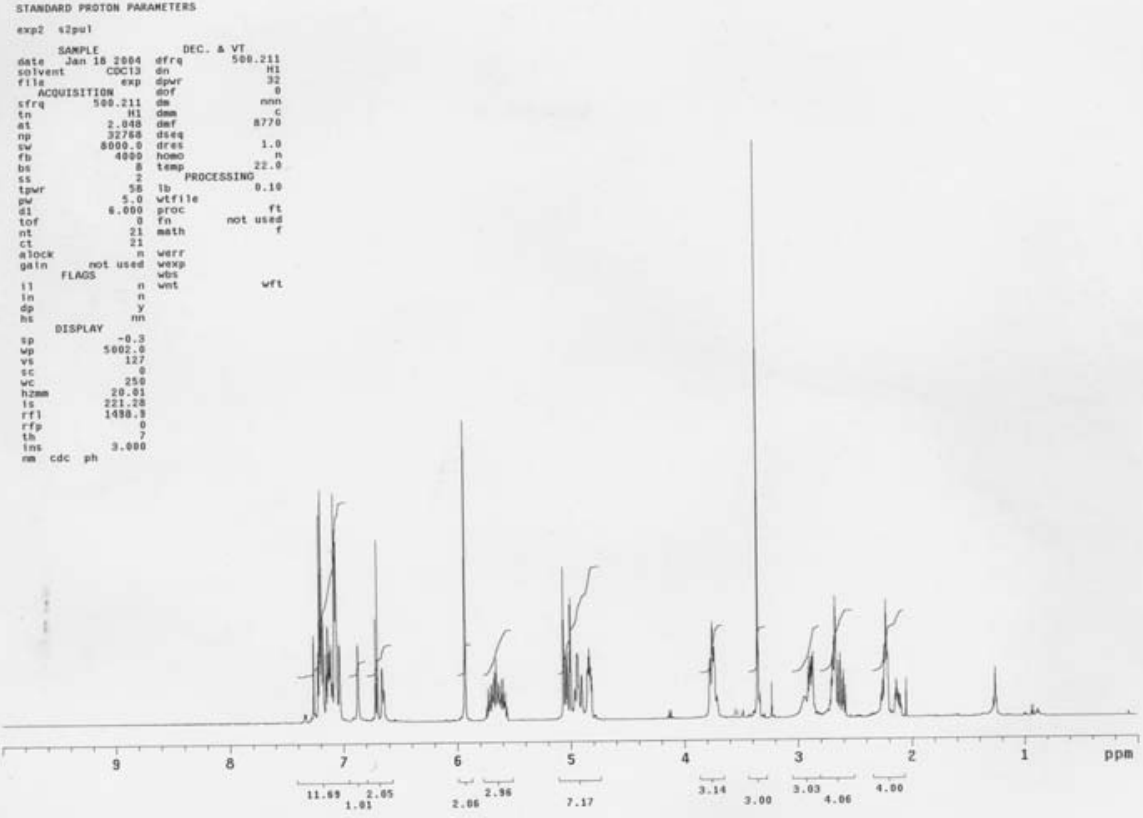

${ }^{1}$ H NMR spectrum of [ [ 34 B]

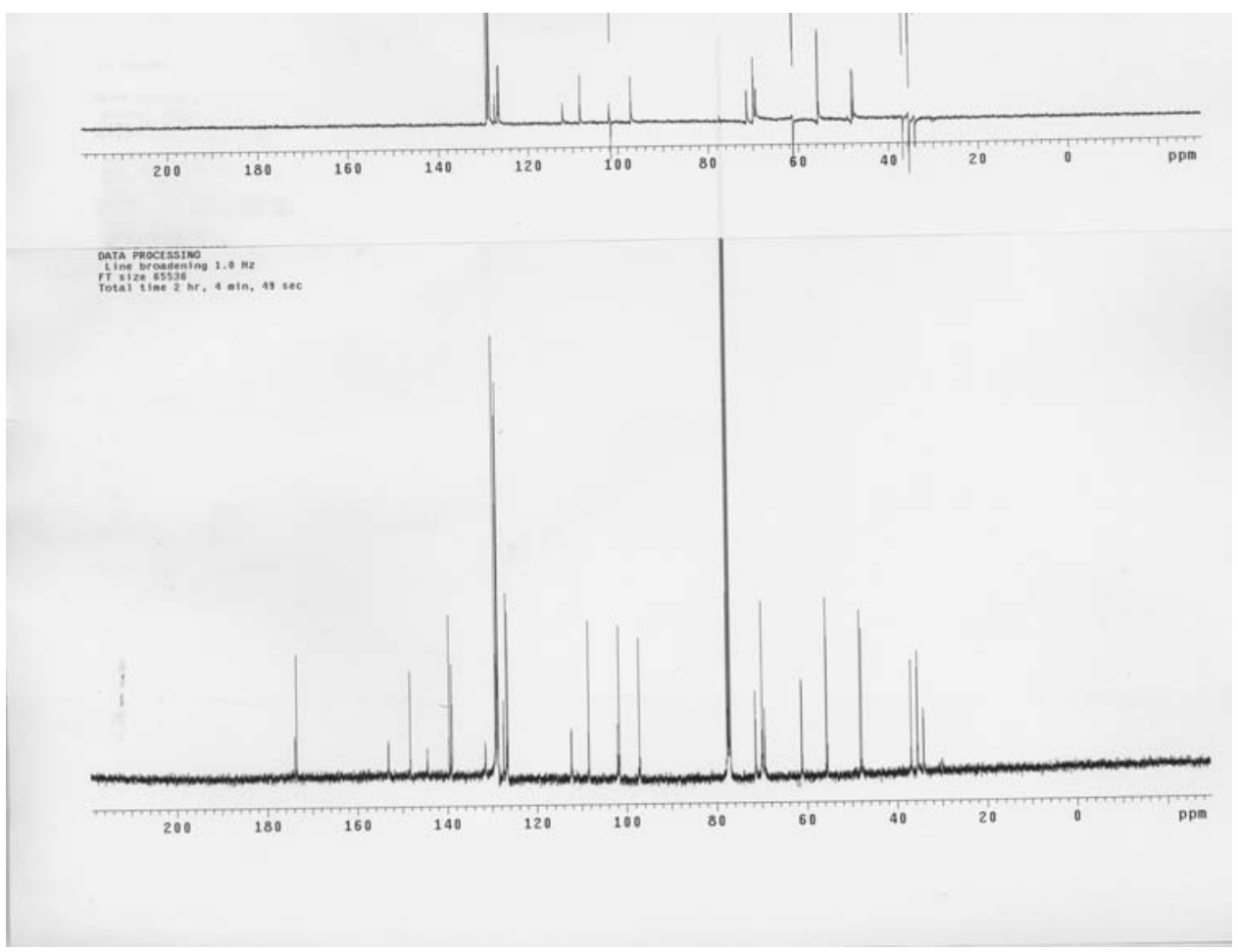

${ }^{13} \mathrm{C}$ NMR spectrum of [I 34 B] 
$0 x p 2$ sip

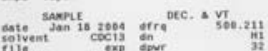

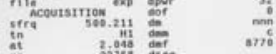

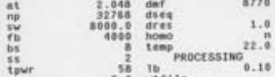

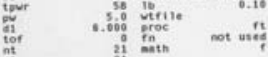

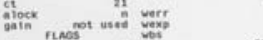

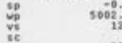

tid

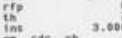

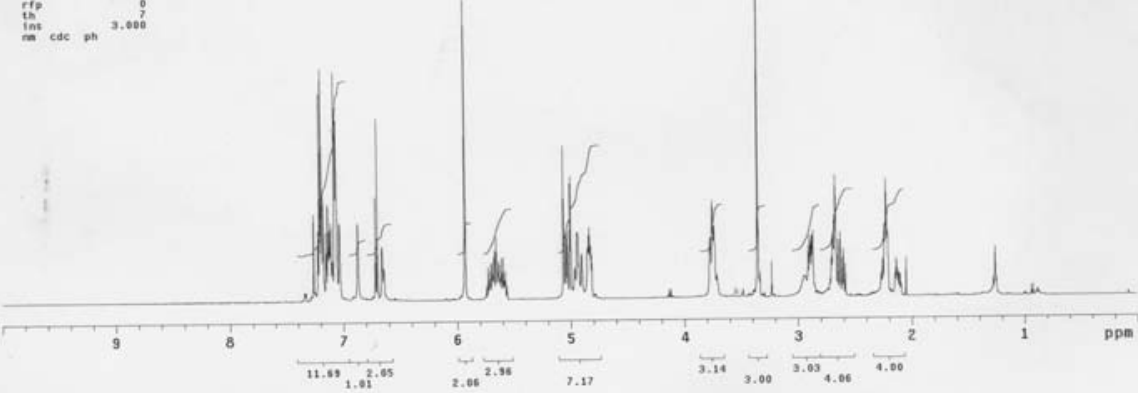

${ }^{1} \mathrm{H}$ NMR spectrum of of [l $\left.34 \mathrm{M}\right]$

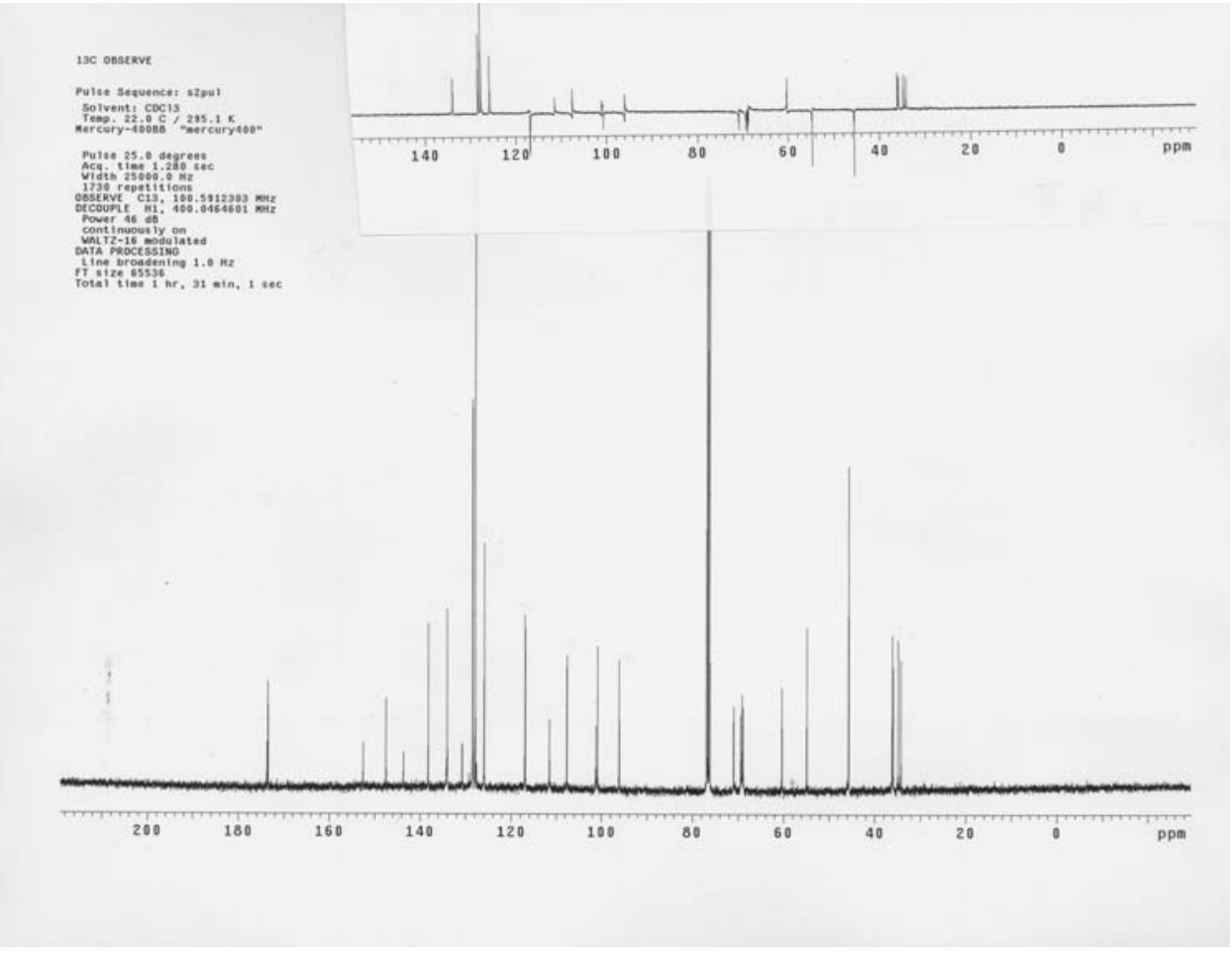

${ }^{13} \mathrm{C}$ NMR spectrum of [ [ $\left.\begin{array}{lll}3 & 4 & \text { M] }\end{array}\right]$ 


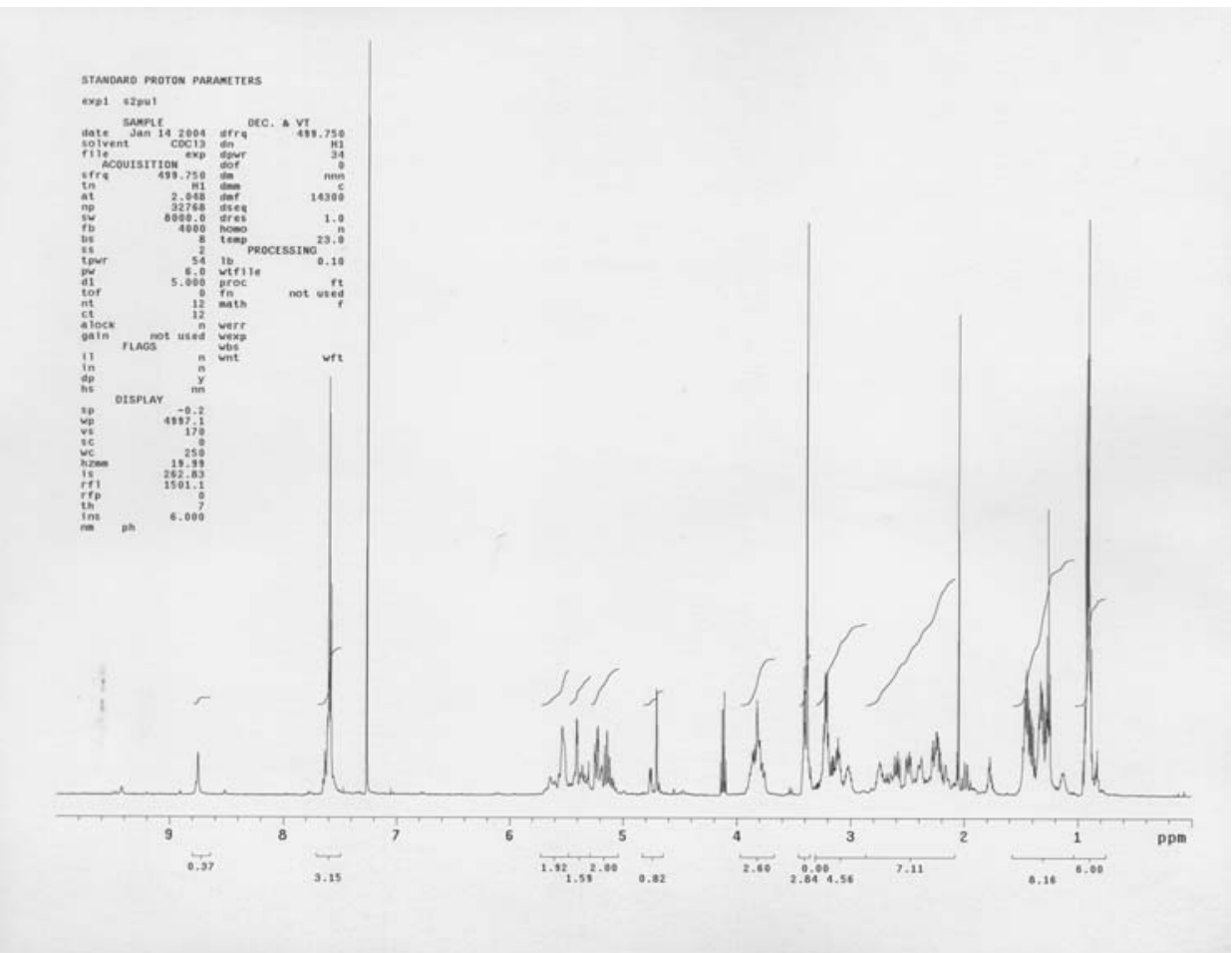

${ }^{1} \mathrm{H}$ NMR spectrum of $\left[\begin{array}{lll}4 & 8 & B\end{array}\right]$

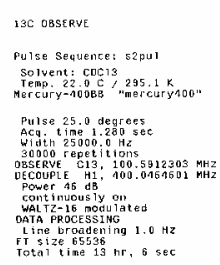

FT $512 e \in 5558$
Total tima $13 \mathrm{hr}, 6$ ses

${ }^{13}$ C NMR spectrum of [III 4 8 B] 


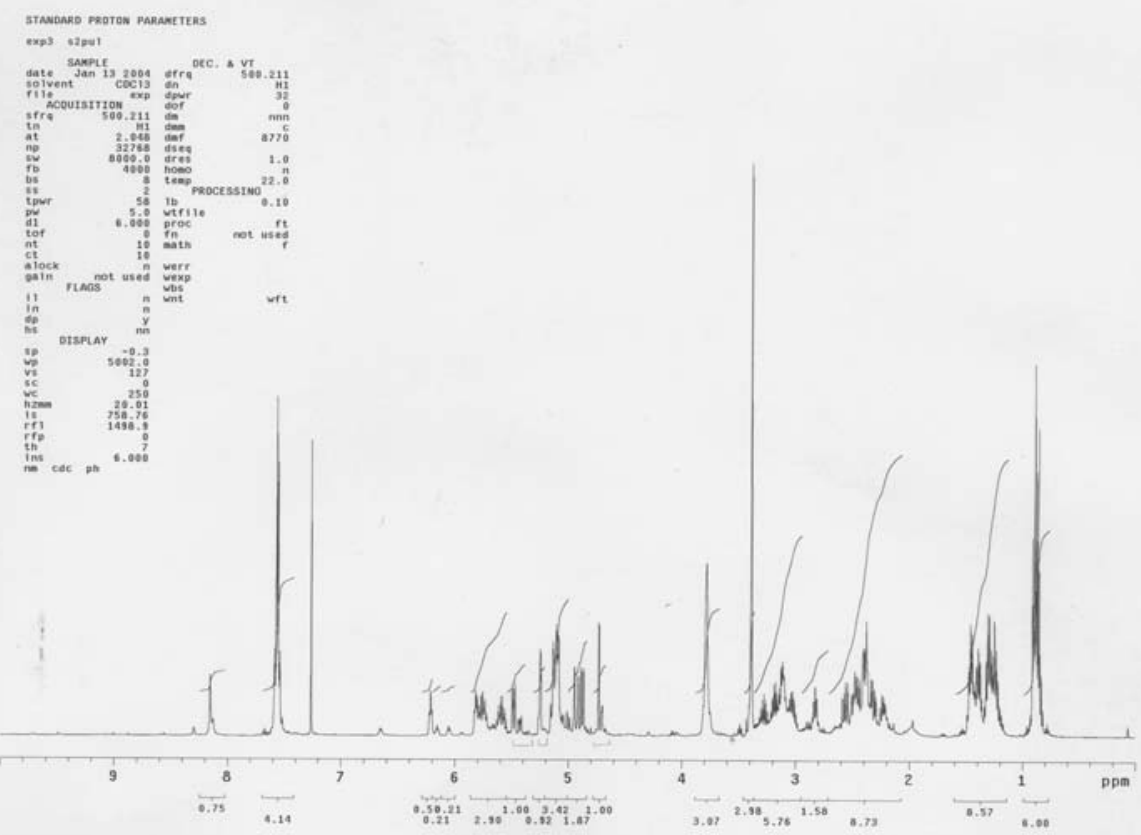

${ }^{1} \mathrm{H}$ NMR spectrum of [III $4 \quad 8$ B]

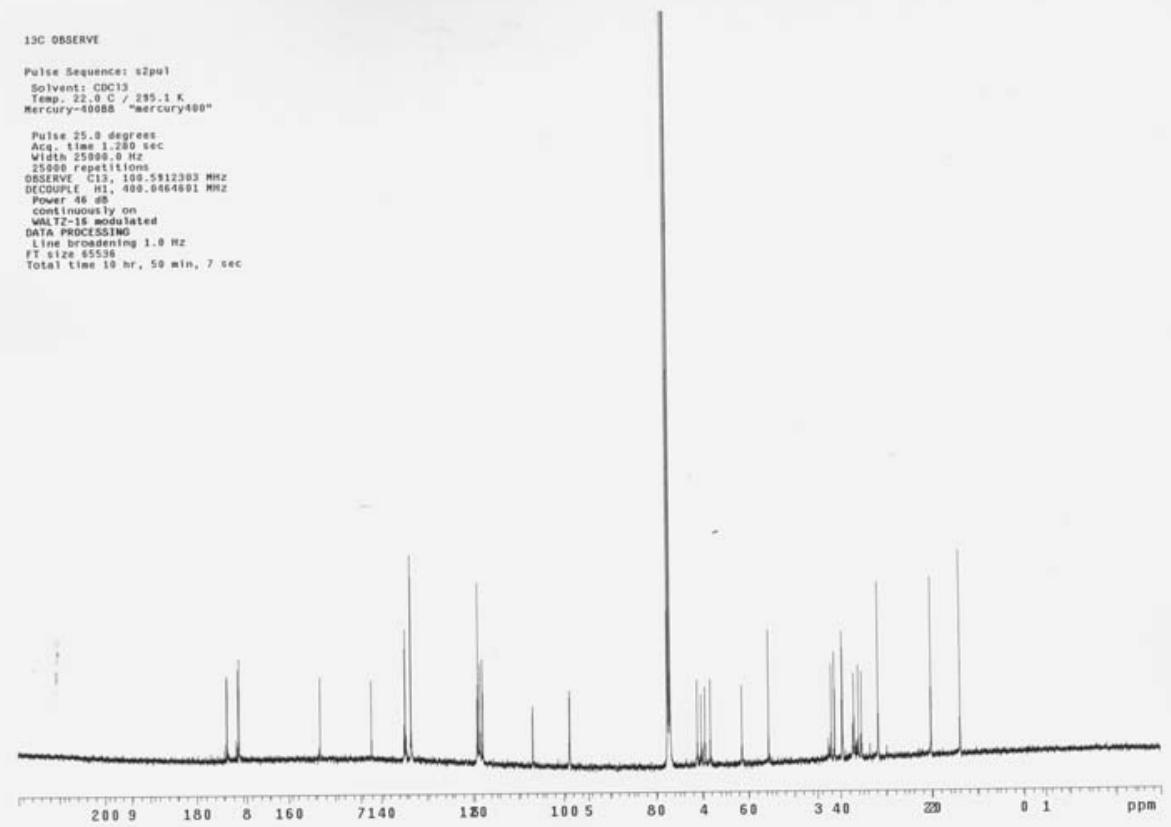

${ }^{13} \mathrm{C}$ NMR spectrum of [III $\left.4 \begin{array}{ll}4 & \mathrm{~B}\end{array}\right]$ 
